NOTE TO USERS

This reproduction is the best copy available. 



\title{
A Channel Based Fair Scheduling Scherr: for Downlink High Speed Data in CDMA Networks
}

\author{
by \\ Ying Zhao \\ A thesis \\ presented to Ryerson University \\ in partial fulfillment of the \\ requirement for the degree of \\ Master of Applied Science \\ in the Program of
}

(Bachelor of Engineering in Electrical Engineering, China, 1996)

Electrical and Computer Engineering.

Toronto, Ontario, Canada, 2003

(C) Ying Zhao, 2003

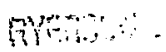


UMI Number: EC52903

\section{INFORMATION TO USERS}

The quality of this reproduction is dependent upon the quality of the copy submitted. Broken or indistinct print, colored or poor quality illustrations and photographs, print bleed-through, substandard margins, and improper alignment can adversely affect reproduction.

In the unlikely event that the author did not send a complete manuscript and there are missing pages, these will be noted. Also, if unauthorized copyright material had to be removed, a note will indicate the deletion.

(B)

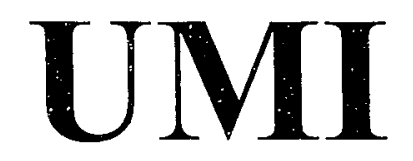

UMI Microform EC52903

Copyright 2009 by ProQuest LLC.

All rights reserved. This microform edition is protected against unauthorized copying under Title 17, United States Code.

\section{ProQuest LLC}

789 E. Eisenhower Parkway

PO Box 1346

Ann Arbor, MI 48106-1346 


\section{Instructions on Borrowers}

Ryerson University requires the signatures of all persons using or photocopying this thesis. Please sign below, and give address and date.

Reproduced with permission of the copyright owner. Further reproduction prohibited without permission. 


\begin{abstract}
Ying Zhao, Master of Applied Science, Electrical \& Computer Engineering, Ryerson University

High speed data transmission in wireless networks demands better radio resource management schemes. The research work for this thesis considers packet scheduling in downlinks of a cellular CD̈MA system for delay-tolerant applications.

In this thesis, a packet scheduling scheme is proposed that attempts to provide fair allocation of individuals throughout and obtain relatively high system throughput. It is based on combined consideration of channel conditions, required throughput and achieved average throughput. A priority factor and system tolerance factor are introduced. We confirmed the tradeoff between system throughout (i.e., efficiency) and individual throughput (i.e., fairness) by both analysis and simulation.

Relative performance between the proposed scheme and traditional schemes is evaluated through simulation to confirm the analytical observations. The sensitivity of system tolerance factor towards efficiency and fairness was also investigated. Overall, the proposed scheme performs between absolute unfairness scheme and absolute fairness scheme in terms of system throughput and fair allocation of individual throughput.
\end{abstract}

iv

Reproduced with permission of the copyright owner. Further reproduction prohibited without permission. 


\section{Acknowledgments}

I wish to express my deepest gratitude to my supervisor Dr. Alagan Anpalagan for his instruction, intelligence, and inspiration throughout the entire research for this thesis.

I would like to thank the members of my thesis oral exam committee - Dr. X.N. Fernando, Dr. X.P. Zhang, and Dr. S. Krishnan for being interested in my research work and providing constructive suggestions and comments. I would like to say thanks to all WINCORE Lab members for the friend and intellectual discussion.

Above all, I would like to acknowledge the education and support from my parents. Finally and most importantly, my husband with his passionate love and continuous encouragement has been extraordinary. 


\section{Contents}

1 Introduction 1

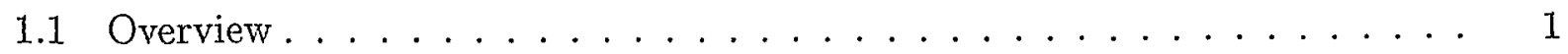

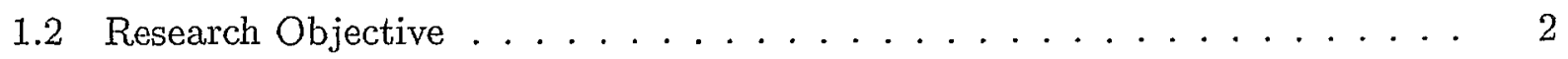

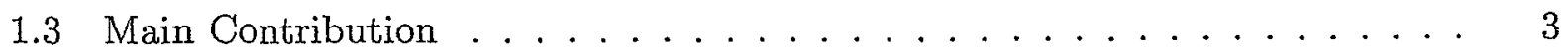

1.4 Thesis Organization . . . . . . . . . . . . . . . . . 4

2 Mobile Communication System $\quad 5$

2.1 Multiple Access Technologies . . . . . . . . . . . . . . . . 6

2.2 Spread Spectrum Communication System . . . . . . . . . . . 10

2.3 Radio Channels . . . . . . . . . . . . . . . . . . . . . 14

2.4 Radio Links and Control . . . . . . . . . . . . . . . . 17

2.5 Scheduling and Quality of Service in Wireless Networks . . . . . . . . . . 19

3 Related Work in Downlink Scheduling Schemes $\quad 21$

3.1 Channel-Based Only Scheduling Scheme (CBO) . . . . . . . . . . 22

3.2 Random Scheduling Scheme (RS) . . . . . . . . . . . . 25

3.3 Round Robin Scheduling Scheme (RR) . . . . . . . . . . . . 26

3.4 Channel-Based Proportional Fair Scheduling Scheme (CB+PF) . . . . . 29

3.5 Modified Channel-Based Proportional Fair Scheduling Scheme (MCB+PF) . 31

4 Proposed Fair Scheduling Scheme (CB+MS+FS) 34

4.1 Fair Scheduling Problem . . . . . . . . . . . . . . . . 34

4.1.1 Definition of Fairness . . . . . . . . . . . . . 34

Vi

Reproduced with permission of the copyright owner. Further reproduction prohibited without permission. 
4.1 .2 Motivation . . . . . . . . . . . . . . . 36

4.2 Proposed Algorithm . . . . . . . . . . . . . . . 36

4.2 .1 Priority Factor $\ldots \ldots \ldots \ldots \ldots \ldots \ldots \ldots \ldots$

4.2 .2 Achieved Average Rate . . . . . . . . . . . . . . 44

4.2 .3 Scheduling Indicator $\ldots \ldots \ldots \ldots \ldots$. . . . . . . . .

4.3 Analysis . . . . . . . . . . . . . . . . 4

4.3 .1 System Throughput. . . . . . . . . . . . . . 4

4.3.2 Standard Deviation of Individual Throughput . . . . . . . . . 4t

4.3 .3 System Tolerance Factor - L . . . . . . . . . . . . . . . 4 4 \&

4.3.4 Signal Quality - SIR . . . . . . . . . . . . . 5i

4.4 Comparison with Existing Schemes . . . . . . . . . . . . . 5

4.4 .1 Complexity . . . . . . . . . . . . . 5

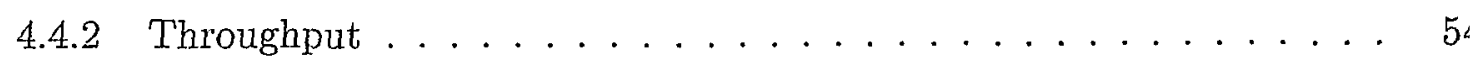

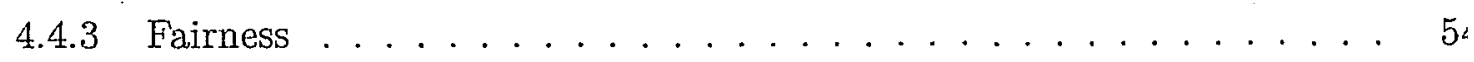

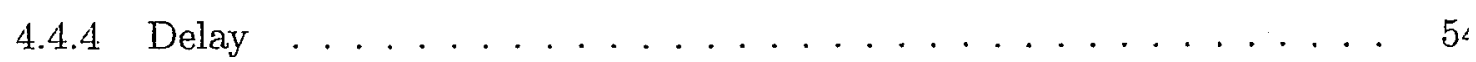

$5 \quad$ Simulation and Results $\quad 5 €$

5.1 Simulation Model . . . . . . . . . . . . . . . . . . 5 5

5.1 .1 Simulation Setup . . . . . . . . . . . . . . 5 t

5.1 .2 Simulation Process . . . . . . . . . . . . . . 5 5

5.2 Simulation Results . . . . . . . . . . . . . . 6 :

5.2 .1 System Throughput. . . . . . . . . . . . . 6 6:

5.2 .2 Standard Deviation of Individual Throughput . . . . . . . . . . 6 6

5.2 .3 Frequency of Scheduling . . . . . . . . . . . . 6 6

5.2 .4 Individual Throughput . . . . . . . . . . . . . . . 7 1

5.2 .5 System Tolerance Factor - L . . . . . . . . . . . . . . 7 :

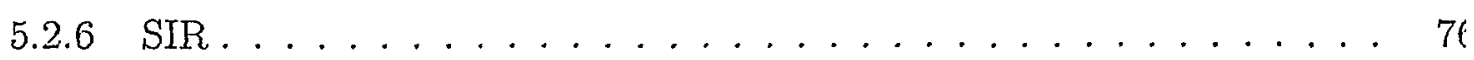

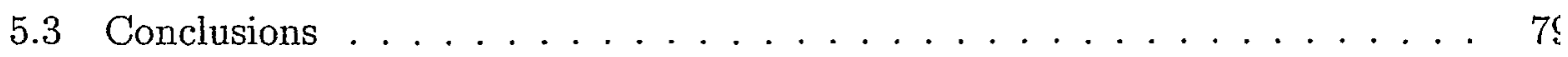

vii

Reproduced with permission of the copyright owner. Further reproduction prohibited without permission. 
$6 \quad$ Summary and Future Extensions $\quad 81$

6.1 Summary . . . . . . . . . . . . . . . . . 81

6.2 Future Work and Practical Implementation . . . . . . . . . . . 82

$\begin{array}{ll}\text { Bibliography } & 83\end{array}$

viii

Reproduced with permission of the copyright owner. Further reproduction prohibited without permission. 


\section{List of Figures}

2.1 Generations of Mobile Communication Systems . . . . . . . . . . 6

2.2 FDMA versus TDMA versus CDMA . . . . . . . . . . . . 7

2.3 Frequency Allocation in FDMA . . . . . . . . . . . 8

2.4 Slot Allocation for 3-slot TDMA . . . . . . . . . . . . . 9

2.5 Direct Sequence Spread Spectrum Approach. . . . . . . . . . . . . . . 12

2.6 Frequency Hopping Sequence. . . . . . . . . . . . . . . 13

2.7 Frequency Division Duplex Technique. . . . . . . . . . . . . 14

2.8 Time Division Duplex Technique. . . . . . . . . . . . . . 14

2.9 Downlink Packet Transmission . . . . . . . . . . . . . . . . . 20

3.1 CBO Scheduling Scheme in a Two-Cell System . . . . . . . . . . . 23

3.2 Two Users are Scheduled During 10 Slot Duration. . . . . . . . . . . . 25

3.3 Average SIR for CBO and RS in a Two-Cell Cellular System. . . . . . . . . 28

3.4 Round Robin Slot Assignment Structure. . . . . . . . . . . . . . 29

4.1 Combined Adaptive Tradeoff between Priority Factor and Achieved Average

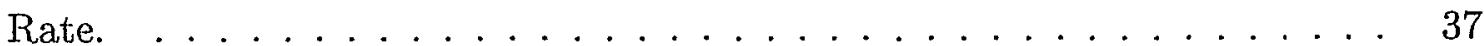

4.2 Combined Adaptive Tradeoff between Normalized Priority Factor and Achieved Average Rate. . . . . . . . . . . . . . . . . . 38

4.3 Time Slot Structure of Method 1 for Updating Achieved Average Rate . . 40

4.4 Time Slot Structure of Method 2 for Updating Achieved Average Rate . . . 41

4.5 Priority Factor with $\mathrm{L}=0 . \ldots \ldots \ldots$

4.6 Priority Factor with $\mathrm{L}=\infty \ldots \ldots \ldots$

ix

Reproduced with permission of the copyright owner. Further reproduction prohibited without permission. 
4.7 Steps of Priority Factor with different value of $L$. . . . . . . . . . . . 51

5.1 A Cellular CDMA System with Two Cells. . . . . . . . . . . . . 56

5.2 System Throughput ....................... 63

5.3 Standard Deviation of Individual Throughput. . . . . . . . . . . 65

5.4 Frequency of Scheduling for Individual User with Distance-Dependent Only. 68

5.5 Frequency of Scheduling for Individual User with Rayleigh Fading (CBO and $\mathrm{CB}+\mathrm{MA}+\mathrm{FS}) . \ldots \ldots \ldots \ldots \ldots \ldots$

5.6 Frequency of Scheduling for Individual User with Rayleigh Fading (RR and

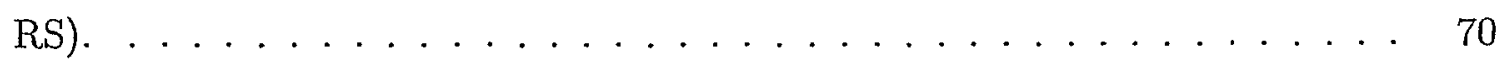

5.7 Individual Throughput with Rayleigh. Fading (CBO and CB+MA+FS). . . 71

5.8 Individual Throughput with Rayleigh Fading (RR and RS). . . . . . . . 72

5.9 System Throughput versus $L$ with Rayleigh Fading. . . . . . . . . . . . . 74

5.10 Standard Deviation of Individual Throughput versus L with Rayleigh Fading. 74

5.11 SIR Distribution in CBO . . . . . . . . . . . . . . . . 77

5.12 SIR Distribution in CB+MA+FS . . . . . . . . . . 77

5.13 SIR Distribution in RS. . . . . . . . . . . . . . . . 78

5.14 SIR Distribution in RR. . . . . . . . . . . . . 78 


\section{List of Tables}

3.1 SIR for CBO and RS Scheduling Scheme in a Two-Cell Cellular System. . . 27

3.2 Adaptive Variable Rate and SIR. . . . . . . . . . . . . . . 30

4.1 Performance Comparison of Different Scheduling Schemes. . . . . . . . . . 55

5.1 Simulation Parameters. . . . . . . . . . . . . . . . . 58

5.2 System Throughput. . . . . . . . . . . . . . . . . 64

5.3 Standard Deviation of Individual Throughput . . . . . . . . . . . . 66

5.4 Percentage of Users Scheduled. . . . . . . . . . . . . . . 71

5.5 System Throughput and Standard Deviation of Individual Throughput with L in Rayleigh Fading . . . . . . . . . . . . . . . . . 75

5.6 Average SIR in a Two-cell System . . . . . . . . . . . . . . . 79

5.7 Overall Summary of Performance Measures in CBO, CB+MA+FS, RR, RS Scheduling Schemes. . . . . . . . . . . . . . . 79 


\section{Chapter 1}

\section{Introduction}

\subsection{Overview}

Future mobile communication systems are expected to provide a broad range of multimedia transmission capabilities. They should also be able to handle a wide variety of services with bit rate requirements ranging from a few kbps to as much as $2 \mathrm{Mbps}$ [2]. CNMA has been proposed as the multiple access technique for the next generation wireless communications systems, as specified in the International Mobile Telecommunication 2000 (IMT2000) proposals. As tetherless communication and computing become more and more ubiquitous, future wibeband CDMA systems are required to efficiently utilize the limited wireless spectrum due to the rapidly growing demands for high data rate services. Taking into account the bursty nature of data traffic and time-varying demand for resources from video traffic, packet transmission over wireless links make it possible to achieve a high statistical multiplexing gain [3].

Packet communication technology, which is well-suited for multimedia communications, copes with the traffic and quality of service (QoS) variations in the downlink wireless communication environment. To simultaneously maximize wireless resource utilization and guarantee QoS satisfaction, packet transmission needs to be scheduled properly in CDMA networks. Being one of the most important components of quality of service provisioning and resource sharing, packet scheduling plays a key role in wireless data networks. A scheduling scheme directly controls the transmission of multimedia payload and allocates the radio resources 
more efficiently as an effective radio resource management technique. Scheduling schemes can help achieve the objective of higher forward link data throughput while maintaining required QoS at the same time. Scheduling algorithms are important components in the provision of guaranteed quality of service parameters such as delay, delay jitter, packet loss rate, or throughput. The design of scheduling algorithms for mobile communication networks is especially challenging given the highly variable link error rates and link capacities [12]. A scheduler operates across different sessions (connections or flows) in order to ensure that reserved throughput and bounds on delays and loss rates are met. The basic function of scheduling algorithm is to select the session whose packet is to be transmitted next which is the main focus of our research work.

Most of the data traffic is non real-time in nature but requires high transmission accuracy. The transmission delay requirement depends on each particular data application. On the other hand, voice and video arc real-time traffic and have strict transmission delay requirements. However, they can tolerate a certain degree of transmission errors. Scheduling scheme plays a prominent role in non real-time applications that can tolerate delay since it can provide high data throughput while maintaining the high transmission quality.

For multimedia services, the resource demand in the reverse link and forward link are generally not the same and are time-varying. A much higher forward rate is required from the access point (base station) than that generated by the access terminal (mobile terminal) in the reverse link in current wireless data networks. In this thesis, we consider forward link only.

\section{$1.2 \quad$ Research Objective}

In order to support high data rate packet services with impending explosion of high speed wireless data communications in third generation (3G) systems, the scarce and limited wireless resources must be efficiently used. Among the existing conventional scheduling schemes, channel-based only (CBO), round robin (RR) and random scheduling (RS), $R R$ is an absolutely fair scheduling scheme since each user will get the same chance to transmit; $\mathrm{CBO}$ is 
definitely unfair scheduling scheme since the user with the best channel quality is scheduled always. A resource allocation scheme which is based only on the best channel quality can maximize the system capacity, but is unfair to the users with adverse channel conditions. CBO utilized system resources efficiently but neglected the fairness for individual users. All of the above scheduling schemes do not consider fairness and system efficiency together. However, it is not easy to meet all of these requirements. That is, there is a trade off between maximizing spectral efficiency and meeting individual user's diverse need.

After studying the existing conventional scheduling techniques and identifying areas for potential research, we focused our research on

1. developing a fair (in terms of achieving equal individual throughput over some time) scheduling scheme that also takes real-time channel conditions into account in the downlink of a cellular CDMA system that supports slotted transmission.

2. Investigating the relative performance of the developed scheme with conventional absolutely fair scheme (i.e.,round robin) and absolutely unfair scheme (i.e., channel based only).

\subsection{Main Contribution}

In the thesis research

- a novel packet scheduling scheme is proposed that is based on combined consideration of real-time channel conditions, requested throughput and achieved average throughput. It attempts to fairly allocate the system throughput among users,

- tradeoff between the system throughput (or efficiency) and individual throughput (or fairness) is confirmed both analytically and by means of simulation,

- sensitivity of the system-defined tolerance factor is analyzed and its flexibility in system operation is noted, 
- performance of the proposed scheme is cvaluated and compared to that of channelbased only and round robin schemes to confirm the analytical observations,

- the balanced performance of the proposed scheme (between fair and efficient approaches) is observed.

\subsection{Thesis Organization}

The rest of the thesis is organized as follows. In the next chapter, we discuss mobile communication system with emphasis on direct sequence spread spectrum communication system. Also briefly discussed are packet transmission and quality of service. In Chapter 3 , the existing packet scheduling schemes are characterized and discussed in detail. Some examples are given to compare the performance among these scheduling algorithms. A fair packet scheduling scheme that take both channel conditions and delivered throughput into account is proposed in Chapter 4. The definition of fairness is given firstly, then approaches to improve fairness are discussed with the analysis of mean of system throughput and variance of individual throughput. Effectiveness of the fair packet scheduling scheme is verified by simulation in Chapter 5. Many performance measures such as, degree of fairness, throughput (both system and individual) and frequency of scheduling are evaluated there. Thesis concludes with a summary of the thesis work followed by future work.

Reproduced with permission of the copyright owner. Further reproduction prohibited without permission. 


\section{Chapter 2}

\section{Mobile Communication System}

Mobile communication era was born in the 1970s with the development of highly reliable, miniature, solid-state radio frequency hardware. The recent exponential growth in cellular radio and personal communication systems throughout the world is directly attributable to new technologies of the 1970s, which are mature today [1]. The future growth of mobile and portable communication systems will be tied more closely to radio spectrum allocations and regulatory decisions which affect or support new services, as well as to technology advances in signal processing, multiple access, and networking. Mobile communication systems are broadly divided into the first $(1 G)$, second $(2 G)$, and third generations (3G), based on whether the system is analog or digital, and they provide voice or multimedia services. Fig. 2.1 summarizes the time frame around when each appeared on the market or is expected to appear in the case of fourth generation $(4 \mathrm{G})$ system. The first generation of mobile system has already been phased out, and the second generation of mobile system has been in operation now for some 10 years and is expected to be replaced by or to be evolved into the third-generation systems, which are presently being rolled out.

In this chapter, we briefly discuss some basic concepts involved in the mobile communication. 


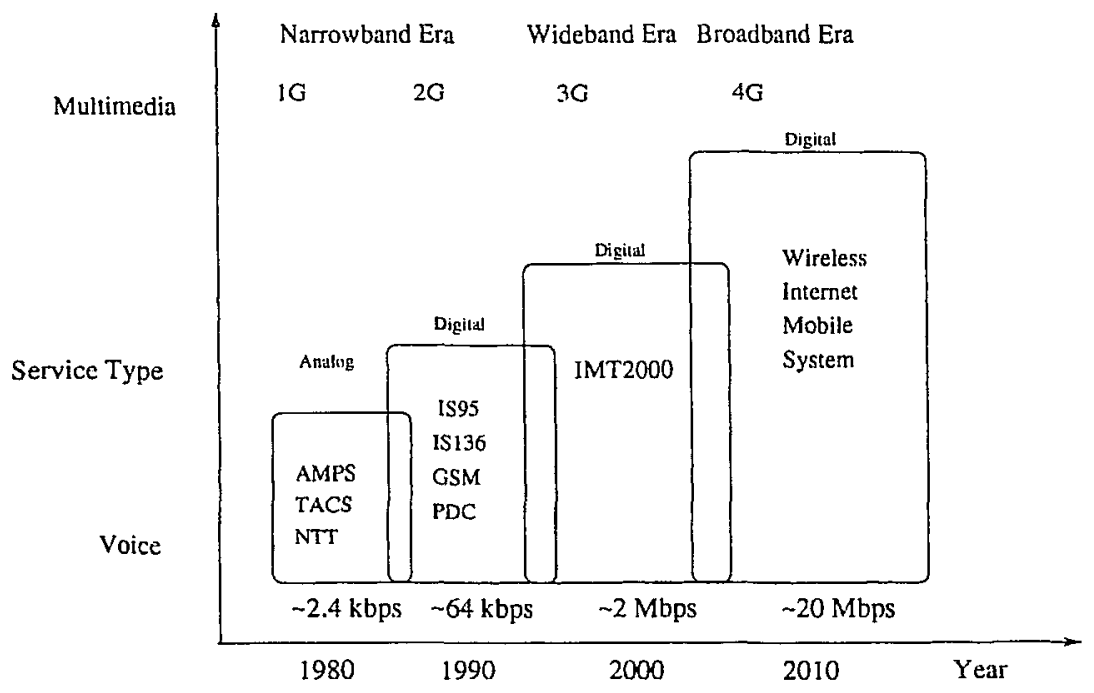

Figure 2.1: Generations of Mobile Communication Systems .

\subsection{Multiple Access Technologies}

A general trend can be observed in the utilized multiple-access scheme: from frequency division multiple access (FDMA) to time division multiple access (TDMA) and code division multiple access (CDMA) as time progresses. FDMA, TDMA and CDMA are mainly utilized in $1 \mathrm{G}, 2 \mathrm{G}$ and $3 \mathrm{G}$ respectively. $1 \mathrm{G}$ systems based on FDMA are primarily analog systems, such as AMPS, and 2G systems are digital. Fig. 2.2 [1] illustrates the difference between these three access technologies. In FDMA systems, a user is assigned an individual frequency band over which it transmits the information and keeps it for the duration of the connection. In TDMA systems, a frequency band is shared between several users, who in turn use the channel for transmission (or reception) of information at well defined time intervals. In CDMA systems, many users use the same frequency band all the time, and each user is distinguished at the transmitter/receiver by a unique spreading code. 


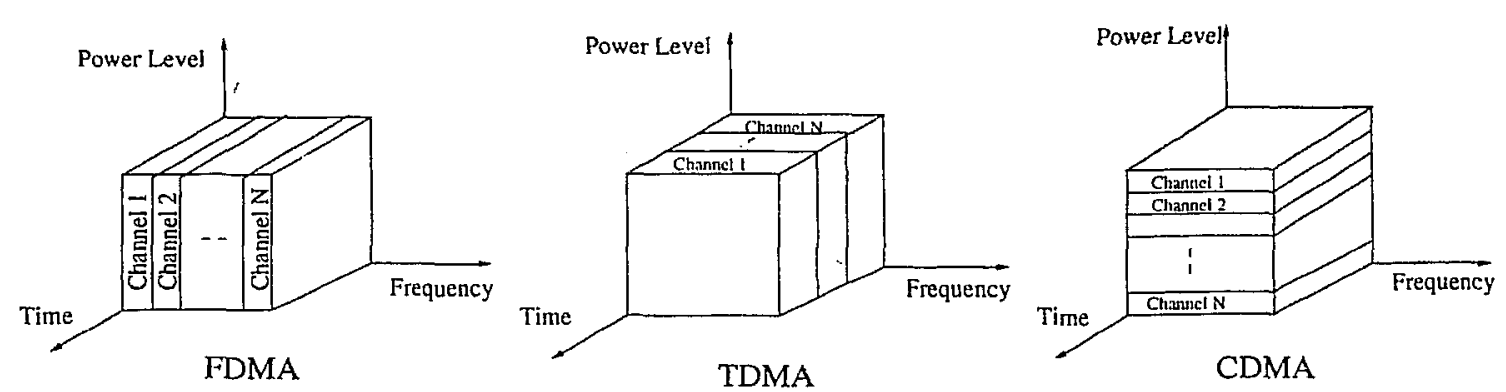

Figure 2.2: FDMA versus TDMA versus CDMA

\section{(A) Frequency Division Multiple Access ( FDMA)}

FDMA system formed the basis for the first widely deployed public cellular systems in North America. In particular, the Advanced Mobile Phone System (AMPS) developed primarily by AT\&T Inc., was initially deployed in North America. This system was deployed in the 800 $\mathrm{MHz}$ band using $30 \mathrm{kHz}$ channel spacing that still exists. ETACS (European Total Access Communication System) was deployed in Europe, with the slight difference from AMPS in that the channel spacing was $25 \mathrm{kHz}$. Similarly, N-AMPS (Narrowband AMPS) was developed by Motorola to work within a $10 \mathrm{kHz}$ channel spacing, thus increasing the original AMPS capacity [3]. These initial FDMA systems are normally referred to as first-generation cellular systems.

FDMA systems generally operate with a base station in each cell with a cluster of cells in coverage area occupying a separate frequency in both the transmit and receive bands. The spectrum allocation for an FDMA system is given in Fig. 2.3. FDMA systems have inherent capacity limitations due to the fact that each frequency channel can be allocated to only one user. In addition, due to the limitation of the frequency reuse factor in a given deployment, co-channel interference is also an issue. 


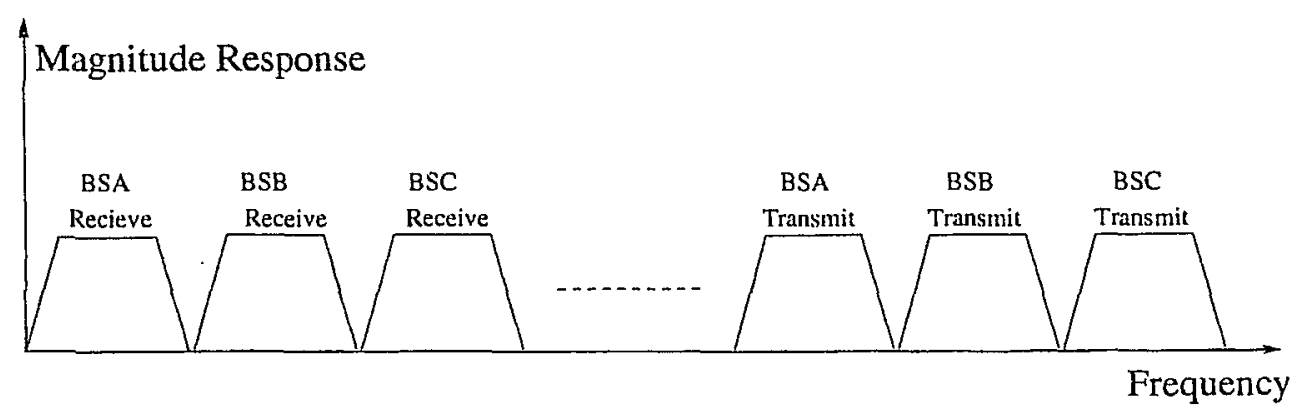

Figure 2.3: Frequency Allocation in FDMA

\section{(B) Time Division Multiple Access ( TDMA )}

The development of the first digital cellular systems is based on TDMA. The $30 \mathrm{kHz}$ channel TDMA system known as US Digital Cellular (USDC) or Digital AMPS (D-AMPS) was developed in the late 1980s. Also in the 1980s, the Group Special Mobile (GSM) in Europe developed a digital TDMA standard to work within $200 \mathrm{kHz}$ channels. The first GSM deployments were in 1991, and the first D-AMPS deployments in North America were in Canada by CANTEL in 1992 [1]. These TDMA systems are also grouped under a general classification for initial digital cellular technologies known as second generation.

TDMA cellular systems utilize the spectrum in a similar manner to FDMA systems, with each base station in a cell occupying a distinct frequency for transmission and reception. However, each of the two frequency bands is also allocated in time to each user in a roundrobin fashion. For example, 3-slot TDMA divides the transmission into three fixed time periods (slots), each of equal duration, with a particular slot assigned for transmission to (or from, in the case uplink) one of three possible users. Fig. 2.4 shows the slot allocation in TDMA system. TDMA system also has capacity limitations which results from the fact that the number of users on any single frequency is restricted by the number of available time slots. This type of approach requires tight synchronization between the mobile station and base station. 


\begin{tabular}{|c|c|c|c|c|c|}
\hline User 1 & User 2 & User 3 & User 1 & User 2 & User 3 \\
\hline- Slot & - Slot - & $\leftarrow$ Slot $\longrightarrow$ & Slot & Slot & $\leftarrow$ Slot $\rightarrow$ \\
\hline
\end{tabular}

Figure 2.4: Slot Allocation for 3-slot TDMA

\section{(C) Code Division Multiple Access (CDMA)}

CDMA is a multiple access technology whereby each user is assigned a unique and high frequency code. In a CDMA system, frequency reuse limitations seen in FDMA and TDMA systems are not quite so critical, as multiple mobile stations and base stations can occupy the same frequencies at once and interference suppression is achieved by spreading the signal.

CDMA system has been considered as an attractive alternative to the existing FDMA technologies and TDMA systems that have been in use during the last two decades. In FDMA and TDMA systems, mobiles can not seemingly roam from one base station to another base station since the neishboring base station uses different frequency spectrum to avoid cochannel interference. In such systems, a group of channels is reused sufficient distance apart which is defined by the frequency reuse factor; which places a practical limit on the minimum number of neighboring base staticns that may use same frequencies. CDMA, on the band, can avoid this type of problem due to the fact that all base stations within a network can use the same frequency and any interference that one CDMA base station imposed on another can be suppressed by spreading the signal. The another advantage of CDMA system is to use soft handoff, which entails the mobile being in simultaneous communication with more than one base station. Once the mobile roams from one base station to another, there is no need to suspend communication with either 1 ase station in the process. This feature also leads to some diversity benefits and potentially lowers the probability of dropped calls as a result of mobility.

Qualcomm Inc. developed the first CDMA cellular system for wide spread deployment 
in the early 1990s, culminating with the standardization of Qualcomm's CDMA solution by the Telecommunication Industry Association (TIA) in 1992 [2]. More recently, CDMA has formed the basis for enhancing cellular systems around the world. Third generation systems are developed to improve wireless multimedia services to cellular subscribers. Three primary technologies were approved in 1998 by the ITU as third generation technologies [3]:

1. Wideband CDMA, developed by the European Telecommunication Standardization Institute (ETSI).

2. CDMA2000, developed by TIA. which is backward-compatible to IS-95.

3. EDGE (Enhanced Data rates for GSM Evolution) which was co-sponsored by ETSI and TIA.

We consider CDMA system in our research work since CDMA has become the most attractive multiple access technology in $3 \mathrm{G}$ and future mobile communication systems. More discussion on CDMA system follows in the next section.

\subsection{Spread Spectrum Communication System}

Since the late 1940s, spread spectrum technology has been used for military applications. A spread spectrum system operates by transforming the narrow band information of an individual user into wideband information by using high frequency codes, each unique for that particular user.

Spread spectrum system has the following two criteria [3]:

1. The transmission bandwidth is much greater than the bandwidth (or rate) of the information being sent.

2. The signal energy is spread on a very wide bandwidth, transmits the expanded signal, and then, at the receiver, despreads the expanded signal back into the original bandwidth. 
Spread spectrum techniques provide an excellent immunity to multipath interference and robust multiple access capability. It allows transmission to be hidden within background noise. Spread spectrum technologies are very bandwidth efficient in a multiple environment. There are two most widely used approaches to implement spread spectrum systems: frequency hopped spread spectrum and direct sequence spread spectrum. For completeness, we discuss both of these approaches next though direct sequence spread spectrum is considered in our work.

\section{(A) Direct Sequence Spread Spectrum (DSSS)}

Direct sequence spread spectrum systems form the basis for the most widely used CDMA standards for public wireless systems today. In DSSS, the carrier is modulated by a digital code also known as pseudo-random (PN) code in which the code bit rate is much larger than the information signal bit rate. DSSS system is a wideband system in which the entire bandwidth of the system is available to each user. A system is defined as a DSSS system if it satisfies the following requirement [5]:

1. The spreading signal has a bandwidth much larger than the minimum bandwidth required to transmit the desired information.

2. The spreading of the data is performed by means of a spreading signal, often called a code signal. The code signal is independent of the data and of a much higher rate than the data signal.

3. At the receiver, despreading is accomplished by the cross-correlation of the received spread signal with a synchronized replica of the same signal used to spread the data.

Fig. 2.5 shows the direct sequence spread spectrum approach, where $T_{c}$ is chip time duration, $1 / T_{c}$ is chip rate, and $T_{0}$ is the maximum output period of waveform, $n$ is the number of stages in the shift register, $2^{n}-1$ is the maximum period of all generated sequences. 


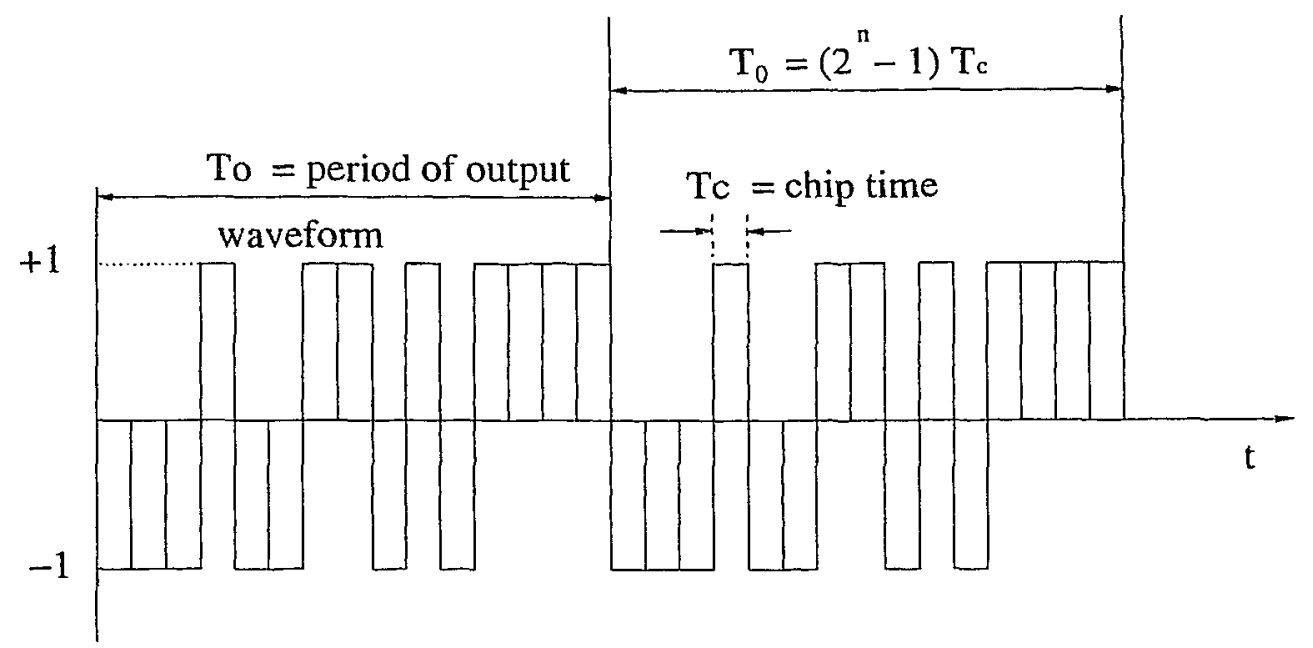

Figure 2.5: Direct Sequence Spread Spectrum Approach.

\section{(B) Frequency Hopping Spread Spectrum (FHSS)}

In FHSS, the carrier frequency is shifted in discrete increments in a pattern generated by a code sequence and the signal frequency remains constant for a specified time duration. CDMA using frequency hopping involves a user transmitting over multiple frequencies consecutively in time in a pseudo random manner. Pseudo random in this case refers to the fact that the sequence of transmission frequencies is known at the transmitter and receiver, but appears random to any other receiver. An example of a frequency hopping sequence is given in Fig.2.6. FHSS can be a fast-hop or a slow-hop system. Slow-hopping systems involve changing of frequencies at a slower rate than the information bit rate, where fast-hopping requires much faster change of the transmission frequency than the information bit rate. Frequency hopped systems are limited by the total number of hopping frequencies available. If two users hop to the same frequency at once, they will interfere with one another.

\section{Duplexing Technique}

In any communications systems, it is often desirable to allow the subscriber to send information simultaneously to the base station and receive information from the base sta- 


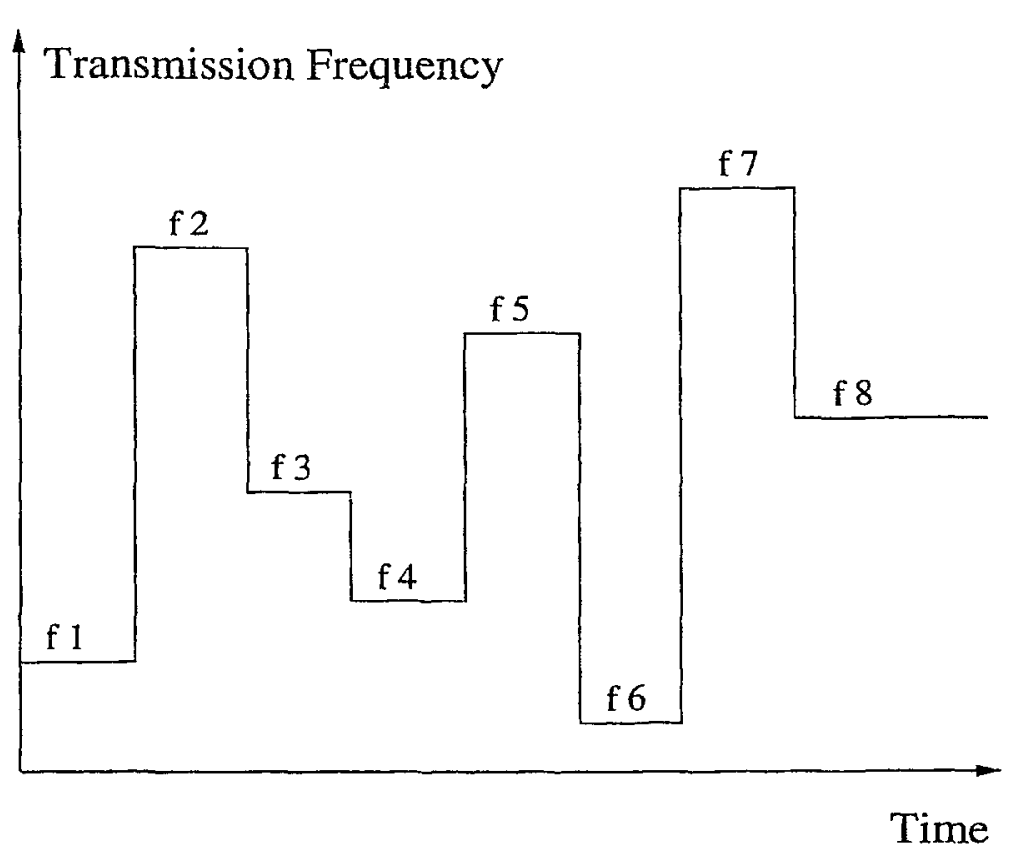

Figure 2.6: Frequency Hopping Sequence.

tion. This effect is called duplexing and is generally required in wireless telephone systems. Duplexing may be done using frequency or time domain techniques.

Frequency division duplexing (FDD) provides two distinct bands of frequencies for every user. In FDD, any duplex channel actually consists of two simplex channels, and a device called a duplexer is used inside each subscriber unit and base station, to allow simultaneous radio transmission and reception on the duplex channel pair as shown in Fig. 2.7. The frequency split between the forward and reverse channel is constant throughout the system, regardless of the particular channel being used. IS-95 and WCDMA are examples of CDMAFDD systems.

Time division duplexing (TDD) uses time instead of frequency to provide both a forward and reverse link which is shown in Fig. 2.8. TDD systems accomplish two-way communication by allowing each user to communicate over the same frequency band by alternately transmitting and receiving. This is accomplished by sharing the channel between the uplink and downlink transmissions by way of allocating certain times for each transmission and 


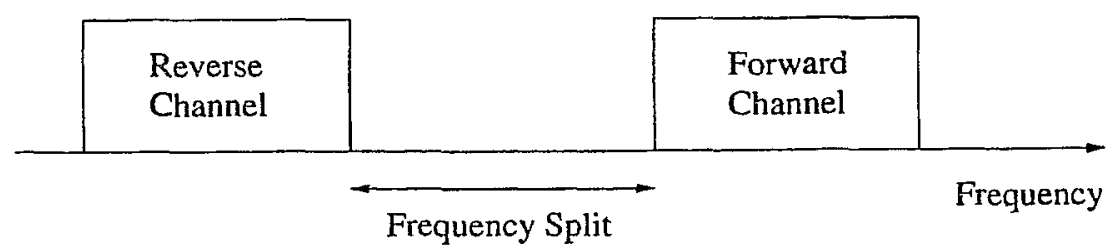

Figure 2.7: Frequency Division Duplex Technique.

switching alternately between the two links. TDD allows communication on a single channel and simplifies the subscriber equipment since a duplexer is not required.

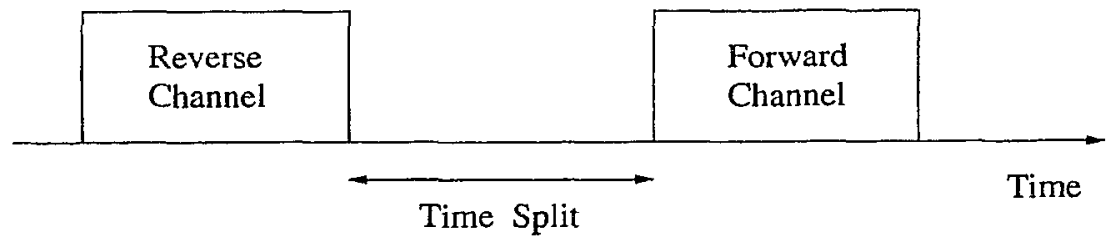

Figure 2.8: Time Division Duplex Technique.

In a TDD system both uplink and downlink transmit in the same frequency band and therefore a frequency guardband is not necessary. However, a time guard at the base station and a smaller guard time at the mobile terminal are necessary to prevent interference between the uplink and downlink transmission.

Our work involves scheduling a packet in each slot in a DSSS CDMA network. Both TDD and time-slotted FDD systems can use our proposed scheduling scheme.

\subsection{Radio Channels}

The radio channel places fundamental limitations on the performance of wireless communication systems. The transmission path between the transmitter and the receiver can vary from simple line-of-sight to one that is severely obstructed by buildings, and mountains, 
etc. Unlike wired channels that are mostly stationary and predictable, radio channels are extremely random, even the speed of motion impacts how rapidly the signal level fades as a mobile terminal moves in space. Modeling the radio channel has is typically done in a statistical manner based on measurements.

\section{Free Space Propagation}

The model of free space treats the region between the transmit and receive antennas as being free of all objects that might absorb or reflect radio frequency energy. It also assumes that, within this region, the atmosphere behaves as a perfectly uniform and non-absorbing medium. The received power expressed in terms of transmitted power is attenuated by a factor, $L_{s}(d)$, where this factor is called free space path loss. For example,

$$
\begin{aligned}
L_{s}(d) & =\left(\frac{4 \pi d}{\lambda}\right)^{\alpha} \\
& =\left(\frac{K}{d^{\alpha}}\right)^{-1}
\end{aligned}
$$

In (2.1), $d$ is the distance between the transmitter and the receiver, $\alpha$ is attenuation factor, $\lambda$ is the wave length of the propagating signal, and $K$ is a constant.

\section{Multipath Propagation}

In a wireless communication system, a signal can travel from transmitter to receiver over multiple reflective paths; this phenomenon is referred to as multipath propagation. The effect can cause fluctuations in the received signal amplitude, phase, and angle of arrival, giving rise to the terminology multipath fading.

\section{Large Scale Fading}

Large scale fading represents the average signal power attenuation or path loss due to motion over large areas. This phenomenon is affected by prominent terrain contours between the transmitter and receiver. The receiver is often represented as being "shadowed" by such prominence. The statistics of large-scale fading provide a way of computing an estimate of 
path loss as a function of distance. This is described in terms of a mean-path loss and a log-normally distribution variation about the mean [14].

For the mobile radio application, the mean path loss, $L_{p}(d)$, as a function of distance, $d$, between the transmitter and receiver is proportional to an $\alpha^{\text {th }}$ power of $d$ relative to a reference distance $d_{0}[4]$.

$$
L_{p}(d) \propto\left(\frac{d}{d_{0}}\right)^{\alpha}
$$

$L_{p}(d)$ is often stated in decibels, as shown below.

$$
L_{p}(d)=L_{s}\left(d_{0}\right)+10 \alpha \log \left(d / d_{0}\right) \quad(\text { in } \mathrm{dB})
$$

The reference distance $d_{0}$ corresponds to a point location in the far field of the antenna. $L_{s}\left(d_{0}\right)$ is typically found through field measurements or calculated using the free space path loss.

The prath loss versus distance expressed in (2.3) is an average, and therefore is not adequate to describe any particular setting or signal path. It is necessary to provide for variations about the mean since the environment of different sites may be quite different for similar transmitter-receiver separations. Measurements have shown that for any value of $d$, the path loss is a random variable having a log-normal distribution about the mean distant-dependent value $L_{p}(d)$. Thus, path loss $L_{p}(d)$ can be expressed in terms of the mean path loss plus a random variable $X_{\sigma}$ [4], as follows:

$$
L_{p}(d)=L_{s}\left(d_{0}\right)+10 n \log _{10}\left(d / d_{0}\right)+X_{\sigma} \quad(\text { in } \mathrm{dB})
$$

where $X_{\sigma}$ denotes a zero-mean Gaussian random variable (in decibels) with standard deviation $\sigma$. The choice of a value for $X_{\sigma}$ is often based on measurement. It is usual for it to take on values as high as 6-10 dB.

\section{Small Scale Fading}

Small scale fading refers to the dramatic changes in signal amplitude and phase that can be experienced as a result of small changes in the spatial separation between a receiver and 
transmitter. Small scale fading manifests itself in two mechanisms, time-spreading of the signal and time-variant behavior of the channel due to motion. For mobile radio applications, the channel is time-variant because motion between the transmitter and receiver results in propagation path changes.

Small-scale fading is often modeled using Rayleigh fading because if the multiple reflective paths are large in number and there is no line-of-sight signal component, the envelop of the received signal is statistically described by a Rayleigh probability distribution function [4]. When there is a dominating non-fading signal component present, such as a line-ofsight propagation path, the small scale fading envelope is described by a Rician probability distribution function. A mobile radio roaming over a large area must process signals that experience both types of fading: small-scale fading superimposed on large-scale fading.

n our work, we consider Rayleigh fading as our multipath small scale fading model along with free-space propagation model.

\subsection{Radio Links and Control}

\section{Forward and Reverse Link}

The forward link provides traffic from the base station to the mobile terminal. There are four types of channels originating from the base station (BS) to mobile station (MS) in a CDMA forward link: pilot, synchronization, paging and traffic channels. The pilot channel provides MS with a beacon, timing and phase reference (for coherent detection), and signal strength for power control [5]. Synchronization channel provides MS with critical time synchronization data. Paging channel contains messages with parameters that MS needs for access and paging. The messages convey system parameters, access parameters, neighbor list, mobile directed paging messages, mobile directed orders, and channel assignment information to MS and it is used to communicate with MS when there is no call in progress. The forward traffic channel is a variable rate channel capable of carrying message data and control data. BS multiplexes MS directed message into forward traffic channel frames. We consider forward link transmission in our work. 
The reverse link provides traffic from the mobile terminal to the base station. There are two types of channels originating from MS in CDMA reverse link: access and traffic channel. The access channel is used to carry MS responses to commands from BS and call origination requests. MS communicates with BS when there is no reverse link channel by using an accoss channel. The reverse traffic channel is a variable rate channel capable of carrying message data and control data. BS multiplexes MS directed message into the reverse traffic channel frame.

\section{Power Allocation and Control}

Power control (or power allocation) is very important in CDMA systems. Powrer allocation is required in the forward links whereas power control is needed in the reverse link. Forward link can also be power controlled in $3 G$ systems. Power control (or power allocation) is the adjustment of the transmitted power level of the MS (or BS). The system limits both forward link and reverse link traffic channel communication to the minimum transmitter power required for the receiving device to accurately retrieve the message. This reduces the amount of interference that a traffic channel imposes on other channels. Forward link power allocation is facilitated by the cell site minimizing its transmit power by evaluating signal quality messages from the mobiles.

In a cellular CDMA system, the total transmitting power from the transmitter is shared by the total mobile terminals which achieve transmission in system. The active mobile terminal is allocated a transmitting power level by BS, and the amount of allocated power is proportional to its required signal quality and required transmitting rate. The allocated power of each active user is also related to individual link gain among others such as QoS requirement. Base station will increase the allocated transmit power to the mobile terminal along with the increase of the distance between the mobile and its home base station. The mobile terminal with higher required signal quality and higher required transmission rate achieves higher allocated power level. 


\subsection{Scheduling and Quality of Service in Wireless Net- works}

Being one of the most important components of quality of service provisioning and resource sharing, packet scheduling plays a key role in wireless data networks. Packet transmission over wireless links takes into account the bursty nature of data traffic [15]. A high-speed CDMA cellular packet system is needed to support multi-media internet access services. The packet data service is different from voice service which needs tight delay bound and constant data rate at talk spurt [13]. Rather than supporting constant data rate, the scheduled packet data service serves the user of good channel condition with high data rate.

Here, we describe a packet transmission system using an example. Fig. 2.9 [6] shows the downlink packet transmission from an access point [6]. The pilot bursts provide the mobile terminals with means to accurately and rapidly estimate the channel conditions. Among other parameters, the mobile terminals estimates the received SIR. The value of the SIR is then mapped to a value representing the maximum data rate that the predicted SIR can support while maintaining a given frame error rate. The channel condition in the form of a data rate request is then fed back to the access point via the reverse link data rate request channel and update as fast as every $1.67 \mathrm{~ms}$. The mobile terminal chooses the receivable highest data rate. The chosen data rate information is transmitted by the access terminal through a data rate control channel to the access point during each time slot. The access point manages the data rate control channels received from access terminals, and determines the order of access terminals to which forward link traffic data is to be transmitted.

Quality of Service (QoS) in wireless networks refers to the ability of the wireless network traffic to request and receive predictable service levels in terms of data throughput capacity (bandwidth), latency variations (jitter), delay, packet loss and error, call dropping, and fairness [17]. In particular, QoS features provide better and more predictable network service by the following methods:

1. supporting dedicated bandwidth for individual connection 


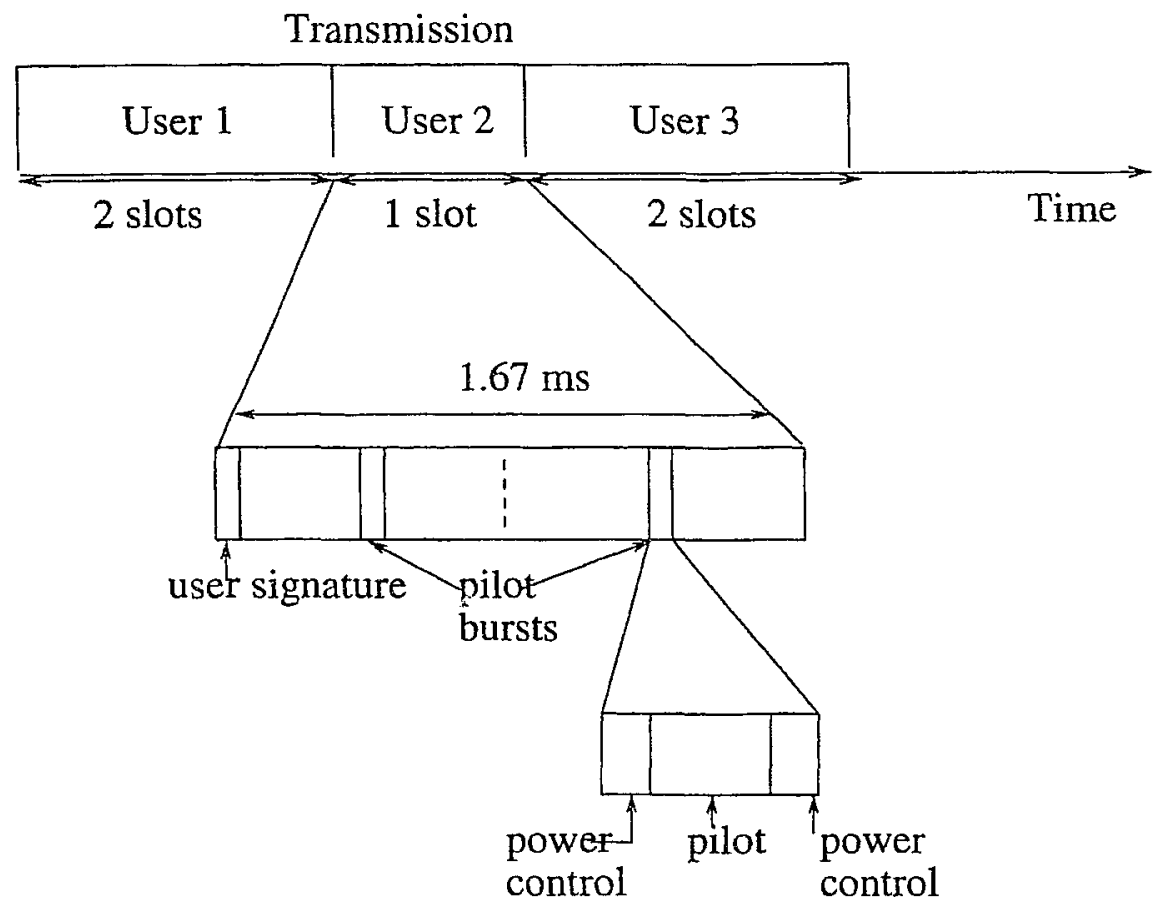

Figure 2.9: Downlink Packet Transmission

2. avoiding and managing network congestion

3. shaping network traffic

4. setting traffic priorities across the network

In the thesis work, (4) is considered as a means to provide QoS (i.e, mainly fairness) it a CDMA network.

Reproduced with permission of the copyright owner. Further reproduction prohibited without permission. 


\section{Chapter 3}

\section{Related Work in Downlink Scheduling Schemes}

In wireless communication systems more attention has been paid to high speed data transmission since more users demand data services recently than voice services. There has been increasing demand for high data transmission services with the explosive growth of traffic from the Internet and E-mail servers etc. that are accessing wireless networks. To support these high data rate services, the limited wireless resources must be utilized efficiently. Among radio resource management techniques, scheduling algorithm has attracted increased attention because it directly controls the transmission of data [12]. Scheduling schemes play a key role in achieving the objective of higher forward link data throughput while maintaining required quality of service (QoS) at the same time.

Among the downlink scheduling algorithms, channel-based only [7] and round robin [9] are two primitive scheduling approaches. In recent development in scheduling scheme [8] and [18], the rate allocated to each mobile terminal varies discretely and depends on each mobile terminal's measured SIR. A scheduler at base station determines the next terminal to be served based on the reported data rate requests from the terminals and the amount of data that has already been transmitted to each terminal. The proposals in [11] and in [19] permit the adjustment of requested rate per mobile terminal based on the real-time channel quality. 


\subsection{Channel-Based Only Scheduling Scheme (CBO) ${ }^{22}$}

Channel-based only scheduling scheme considers the real time channel quality of each mobile terminal in the scheduling of packets. In [7] maximum system throughput is achieved at the expense of fairness, because a single user with the best channel conditon can be allocated all the resources.

We define $S_{j}(n)$ as the scheduled user by base station $j$ with the best channel quality at slot $n$.

$$
S_{j}(n)=\arg \max \left[G_{i j}(n)\right], \quad 0<i \leq M, 0<n \leq N,(i, n) \in I
$$

where $M$ is total user number, $N$ is total slot number, $j$ is index of home base station, $I$ is a positive integer set, $G_{i j}$ is the link gain between user $i$ and its home base station $j$.

If the link condition is stronger for user $i$ for a longer time, user $i$ will be transmitted for a longer time. If the link condition is worse, then the mobile terminal is delayed for some time duration till the channel conditions improve.

\section{An Example: Numerical Results}

We use a numerical example in the following to explain how CBO scheduling scheme works. We assume a cellular CDMA system with two cells, and one base station is located in the center of each cell. Each radio channel suffers propagation loss with an attenuation coefficient of $\alpha=2$, and neither multipath propagation nor shadowing is considered in this example. The system model is graphically shown in Fig. 3.1.

We assume there are only two users which are located in the coverage area of cell $A$, and base station a (BSa) is the home base station of both users. User 1 is located at the distance $d_{1 a}=300$ meters away from BSa and $d_{1 b}=800$ meters away from base station b (BSb). User 2 is located at the distance $d_{2 a}=700$ meters away from $\mathrm{BSa}$ and $d_{2 b}=1600$ meters away from BSb. Base station $\mathrm{b}(\mathrm{BSb})$ is the home base station of neighbor cell $\mathrm{B}$. Therefore, we have link gains between users and their home base station in slot 1 with no 


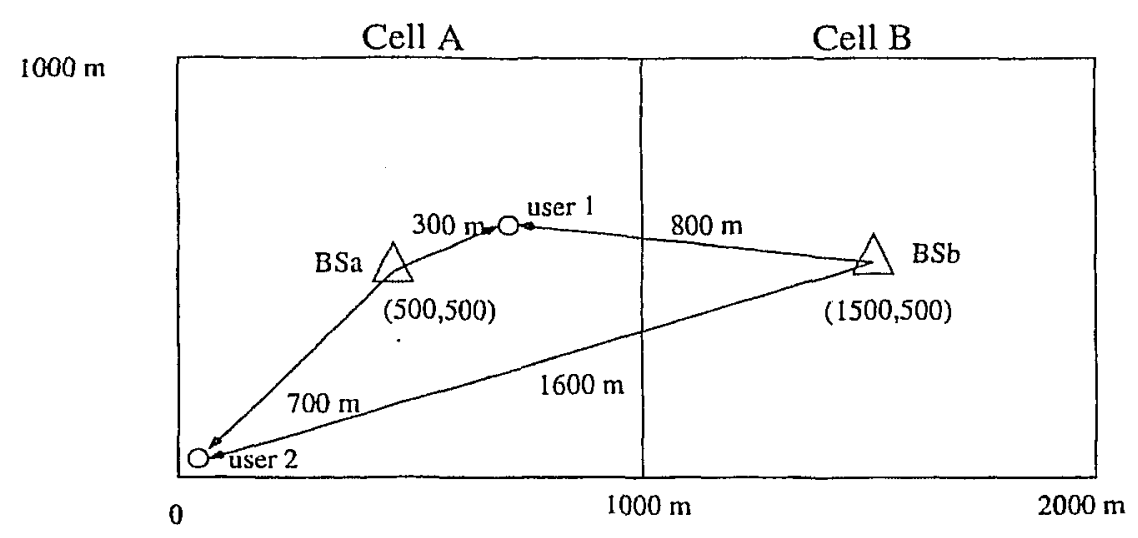

Figure 3.1: CBO Scheduling Scheme in a Two-Cell System

consideration of fading, $G_{1 a}(1)$ and $G_{2 a}(1)$ as:

$$
\begin{gathered}
G_{1 a}(1)=\frac{K}{d_{1 a}^{2}}=\frac{1}{300^{2}} \approx 10^{-5} \\
G_{2 a}(1)=\frac{K}{d_{2 a}^{2}}=\frac{1}{700^{2}} \approx 0.2 \times 10^{-5}
\end{gathered}
$$

where we assumed, for simplicity, $K=1$.

In base station $a$, the user which has the maximum link gain at each slot is scheduled. Since $G_{1 a}(1)>C_{2 a}(1)$, user 1 is scheduled.

In the downlink uncoordinated base stations scenario, scheduling has potential quality degradation problems near the boundaries due to increased interference at high speed transmission as noted in [20]. We can improve this situation through a simple method. A typical consideration is to serve by two low power base stations that are coordinated to avoid potential quality degradation, i.e., only one user is scheduled in each slot, then the interference from intra-cell users or even from neighboring cell users will be decreased tremendously.

Next, we will explain how CBO scheduling scheme affects individual throughput and system average throughput using the transmission rate allocation. Here, we also continue to 
use the previous example. Firstly, we will define the average throughput of cellular CDMA system.

$$
\text { Average Throughput }=\frac{\text { Total Transmission Bits }}{\text { Total Transmission Duration }}
$$

We also define the system average throughput that equals the sum of individual user average throughput:

$$
\text { System Average Throughput }=\sum \text { Individual Average Throughput }
$$

In this numerical example, the locations of two users will not be changed during scheduling duration (with no consideration of shadowing or fading). Let us assume that each base station uses transmission power of $P$ and only one user is scheduled in each slot. The transmission rate of user $i$ is denoted as $R_{i}$. The required signal quality of two users are equal and $E_{b} / N_{0}=\gamma_{i}=10$. The CDMA system bandwidth of $W=1.25 \times 10^{6} \mathrm{MHz}$. We assume 10 slots duration, two users are waiting for being scheduled, and user 1 has link gain $G_{1 a}=10^{-5}$ from base station a, user 2 has link gain $G_{2 a}=0.2 \times 10^{-5}$ from base station a, and $G_{1 a}>G_{2 a}$. Now we calculate the link gain from neighboring base station $b$.

$$
\begin{gathered}
G_{1 b}=\frac{K}{d_{1 b}^{2}}=\frac{1}{800^{2}} \approx 1.6 \times 10^{-6} \\
G_{2 b}=\frac{K}{d_{2 b}^{2}}=\frac{1}{1600^{2}} \approx 0.39 \times 10^{-6}
\end{gathered}
$$

Since the CBO scheduler selects the user which has the best channel condition, the user 1 is scheduled during 10 slots and user 2 is blocked during that time. Therefore, the transmission rate of user 2 is $R_{2}=0$. Next we will compute the transmission rate of the scheduled user 1 . In CBO scheme, the transmission rate is calculated under the assumption that the signal quality is satisfied. $E_{b} / N_{0}$ of user $i$ which is denoted as $\gamma_{i}$ in two cell system can be expressed as :

$$
\gamma_{i}=\frac{W}{R_{i}} \frac{P G_{i a}}{P G_{i b}} .
$$

Hence, we get the expression of $R_{i}$ on the condition that the signal quality is satisfied and calculate $R_{1}$.

$$
R_{i}=\frac{W}{\gamma_{i}} \frac{G_{i a}}{G_{i b}} .
$$




$$
R_{1}=\frac{W}{\gamma_{1}} \frac{G_{1 a}}{G_{1 b}}=\frac{1.25 \times 10^{6}}{10} \frac{10^{-5}}{1.6 \times 10^{-6}}=780 \mathrm{kbps}
$$

The procedure of scheduling is shown in Fig. 3.2 .

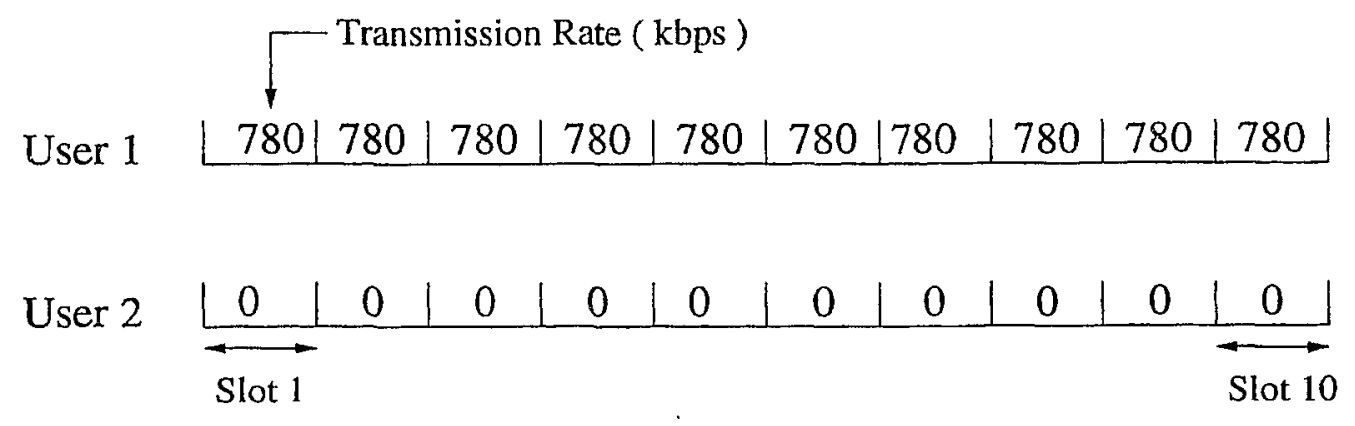

Figure 3.2: Two Users are Scheduled During 10 Slot Duration.

The average throughput of user $i$ is denoted as $R_{i}^{*}$. The average throughput of two users during 10 slots are $R_{1}^{*}=780 \mathrm{kbps}$ and $R_{2}^{*}=0$.

The system average throughput

$$
R^{*}=R_{1}^{*}+R_{2}^{*}=780 \mathrm{kbps}
$$

We find that the system average throughput equals the scheduled user average throughput since the scheduled user keeps transmitting, and the other users are blocked (or delayed) always. It is a kind of unfair situation since the entire system resources are allocated to only one user.

CBO scheduling scheme has good signal quality and can achieve high system average throughput since the channel quality is the only consideration. The fairness is neglected here, because the user with the best channel condition will keep transmitting during the entire scheduling period if there is no shadowing or fading.

\subsection{Random Scheduling Scheme (RS)}

Random scheduling scheme selects mobile terminal to be scheduled randomly in each time slot. We define $S_{j}(n)$ as the scheduled user by base station $j$ with the random selection at 
slot $n$.

$$
S_{j}(n)=\operatorname{random}[\mathrm{M}], \quad 0<n \leq N, n \in I,
$$

where $M$ is total number of active users, random[] picks an integer number randomly between 1 and $M$, and 1 and $M$ are both inclusive.

Next, we will make comparison of SIR in RS and CBO scheduling schemes using the result from simulation which simulated the two scheduling schemes.

\section{An Example: Simulation Results}

In the scheduling simulation, we assume there are two cells in CDMA cellular system, each cell has one base station. In each slot only one user is selected to be scheduled. We assume for each run, the total simulation duration lasts 100 time slots and $T_{s}$ is the length of a slot, $\left(T_{s}=1.67 \mathrm{~ms}\right)$. We calculate average SIR separately for RS and CBO scheduling scheme after 20 runs. The results are shown in Table 3.1 for cell $A$ and $B$ separately.

From the simulation results, we can find that CBO scheduling scheme improves the signal quality compared to RS scheduling scheme since the scheduler in CBO scheme selects the mobile terminal which has the best channel condition while RS scheme selects the mobile terminal randomly in each time slot. The simulation results for the system (i.e, cell $\mathrm{A}$ and B )are also plotted in Fig. 3.3 for 20 runs.

We can find that $\mathrm{CBO}$ scheduling scheme improves SIR by almost $15 \mathrm{~dB}$ compared to RS scheduling scheme.

$\mathrm{RS}$ is a fair scheduling scheme since the scheduler randomly selects the user to be trans-

mitted. RS has low average transmission rate and worse signal quality compared to CBO, but it is easy to be implemented since it need not do the complex operation.

\subsection{Round Robin Scheduling Scheme (RR)}

Round robin is one of the simplest, fairest and most widely used scheduling algorithms, designed especially for time-sharing systems. All active mobile terminals are kept in a circular queue. The RR scheduler cycles through the list of active terminals in a fair manner, 


\begin{tabular}{|c|c|c|}
\hline Run & RS Scheme & CBO Scheme \\
\hline 1 & 4.8 & 23.2 \\
\hline 2 & 10.5 & 29.7 \\
\hline 3 & 6.0 & 25.3 \\
\hline 4 & 9.3 & 17.5 \\
\hline 6 & 10.1 & 23.4 \\
\hline 7 & 13.0 & 21.2 \\
\hline 8 & 14.9 & 25.2 \\
\hline 9 & 4.1 & 29.3 \\
\hline 10 & 2.3 & 23.4 \\
\hline 11 & 9.4 & 24.2 \\
\hline 12 & 8.1 & 25.5 \\
\hline 13 & 6.0 & 21.4 \\
\hline 14 & 12.1 & 20.4 \\
\hline 15 & 13.4 & 23.3 \\
\hline 16 & 17.3 & 28.5 \\
\hline 17 & 10.5 & 19.9 \\
\hline 18 & 4.0 & 33.4 \\
\hline 19 & 8.1 & 21.6 \\
\hline 20 & 14.8 & 24.4 \\
\hline Average SIR & 10.9 & 23.3 \\
\hline & $\mathbf{9 . 4 8}$ & $\mathbf{2 4 . 2}$ \\
\hline
\end{tabular}

Table 3.1: SIR for CBO and RS Scheduling Scheme in a Two-Cell Cellular System. 


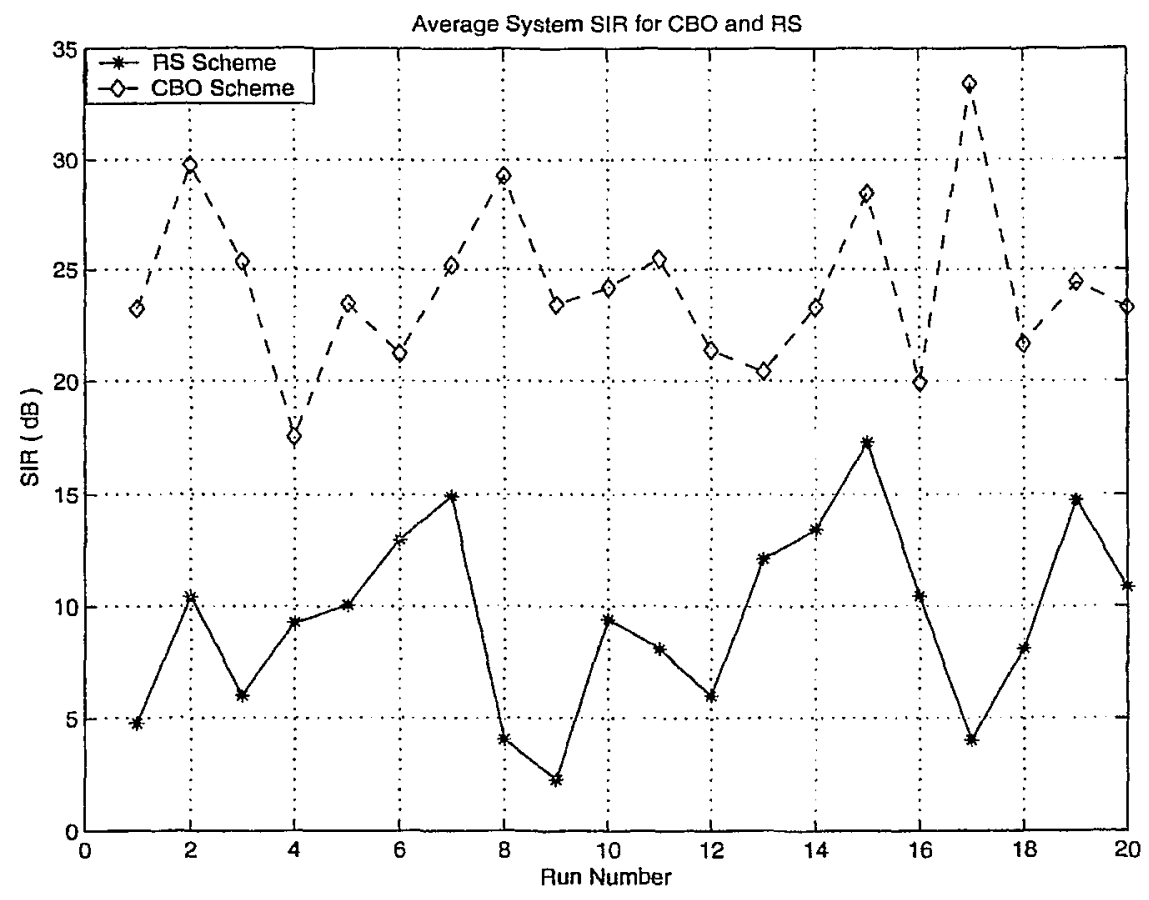

Figure 3.3: Average SIR for CBO and RS in a Two-Cell Cellular System.

allocates the system resources to each mobile terminal on a slot by slot basis. New active mobile terminal are added to the tail of the queue. $R R$ scheduling scheme does not consider the channel quality. If there are $M$ active mobile terminals in the ready queue and the length of each slot is $T_{s}$, then each terminal would ideally get $1 / M$ of the total slots in one second, and each mobile terminal would wait no longer than $M \times T_{s}$ until its next transmission. If the total number of slot is integer times of total user number, then each mobile terminal will be scheduled for same duration (or has the equal number of times scheduled). We call it an absolute fairness scheduling scheme. The performance of the Round Robin scheduling algorithm depends heavily on the length of each slot, $T_{s}$. If $T_{s}$ is very large, $R R$ is similar to the First - Come, First - Served algorithm. If $T_{s}$ is very small, RR approach is called absolute fair sharing.

We define $S_{j}(n)$ as the scheduled user by base station $j$ at slot $n$ under the assumption 
that total slot number and total user number are equal in RR.

$$
S_{j}(n)=n, \quad 0<n \leq N, M=N, n \in I
$$

Fig. 3.4 shows RR slot assignment structure.

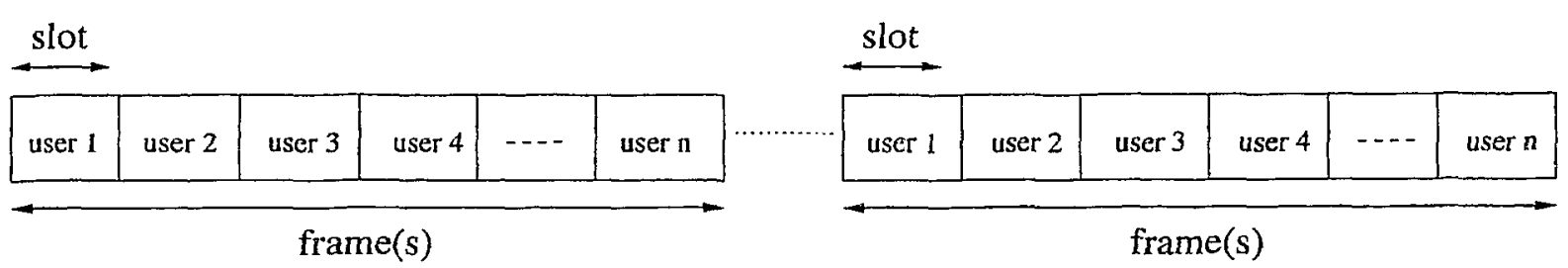

Figure 3.4: Round Robin Slot Assignment Structure.

$R R$ is an absolute fairness scheduling scheme since each user gets same chances to transmit. RR does not consider the channel quality, so the average transmission rate is low and signal quality is worse than in CBO. RR scheduling operation is simple since it does not do complex calculation and comparison.

\subsection{Channel-Based Proportional Fair Scheduling Scheme $(\mathrm{CB}+\mathrm{PF})$}

The channel based proportional fair ( $C B+P F)$ scheduling scheme is based on the requested transmission rate and achieved average transmission rate as proposed in [8]. This algorithm takes channel condition and user request into account implicitly. In the forward link, a selected user is served in a time division multiplexing fashion with full base station power. The forward link of CDMA2000 system consists of a single data channel which is divided into 1.67 ms time slots. The access terminal (AT) constantly measures the channel condition, predicts the SIR and computes the rate that the predicted SIR can support while maintaining a given packet error rate. The access point (AP) on each time slot receives the request data rate information from the AT through the control channel in reverse link. The AP updates and transmits a 4-bit rate control sequence to AT requesting the computed rate every 1.67 


\begin{tabular}{|c|c|c|}
\hline Class & Data Rate (kbps) & SIR (dB) \\
\hline 1 & 38.4 & -12.5 \\
\hline 2 & 76.8 & -9.5 \\
\hline 3 & 153.6 & -6.5 \\
\hline 4 & 307.2 & -4.0 \\
\hline 5 & 614.4 & -1.0 \\
\hline 6 & 921.6 & 1.3 \\
\hline 7 & 1228.8 & 3.0 \\
\hline 8 & 1843.2 & 7.2 \\
\hline 9 & 2457.6 & 9.5 \\
\hline
\end{tabular}

Table 3.2: Adaptive Variable Rate and SIR.

ms. The AP manages the rate control received from ATs, transmits packet data at the rate requested by the AT, and determines the order of ATs to be transmitted by which forward link traffic data is to be transmitted when there are multiple users requesting data. Packet scheduling is excuted at each slot. The request rate mapped from predict SIR is shown in Table 3.2 [10]. Therefore, in CB+PF scheduling scheme, the requested transmission rate is adjusted on the basis of measured SIR. The AP receives a sequence of requested rates from $\mathrm{AT}$ and determines the order of ATs for which forward link traffic data is to be transmitted when there are multiple users requesting data. The $\mathrm{CB}+\mathrm{PF}$ scheduler at base station $j$ computes the average forward link throughput for each user in each slot and sends data to the mobile that has the highest scheduler value $B_{j}(n)$ on decision slot $n[8]$, where

$$
B_{j}(n)=\left[\frac{R_{i}^{r e q}(n)}{R_{i}^{*}(n)}\right], \quad 0<n \leq N, 0<i \leq M,(n, i) \in I
$$

where $R_{i}^{\text {req }}(n)$ is the requested transmission rate by the mobile $i$ in a given slot $n, R_{i}^{*}(n)$ is the achieved average rate of user $i$ upto slot $n$. It should be noted that $R_{i}^{r e q}(n)$ is a function of channel condition which means that requested rate in slot $n$ is dependent on the basis of the channel conditions between user $i$ and its home base station $j$ in slot $n$. The CB+PF scheduler uses rate control mechanism to determine the order and the data rate in which to transmit data to users on the forward link. 
In $\mathrm{CB}+\mathrm{PF}$ scheduling scheme the achieved average rate in each slot is updated as [8]:

$$
R_{i}^{*}(n+1)=(1-1 / T) R_{i}^{*}(n)+1 / T \times R_{i}(n)
$$

where $T$ is the window size constant. The scheduler updates the average transmission rate $R_{i}^{*}(n+1)$ in each slot for each mobile on the basis of the achieved average rate and the transmission rate in slot $n$, then assigns data flow to the mobile that has the highest scheduler value on each decision slot. If the mobile is chosen in slot $n$, the achieved average transmission rate $R_{i}^{*}(n+1)$ for the chosen mobile increases since the chosen mobile keeps transmitting data during its scheduled slot, so the value of scheduler is reduced in slot $n+1$.

$\mathrm{CB}+\mathrm{PF}$ scheme takes both the request rate and acheived average rate into account. The user with high request rate and low average rate will be selected to be transmitted. So the system average throughput is superior in taking advantage temporal variations of channel condition than in RR or RS but CBO.

\subsection{Modified Channel-Based Proportional Fair Schedul- ing Scheme $(\mathrm{MCB}+\mathrm{PF})$}

A modified channel-based proportional fair scheduling scheme was introduced in [9]. The main idea here is that the requested data rate reflects the current channel condition and the average requested rate can reflect the average of channel condition in which mobile is involved. The sclieduler in $\mathrm{MCB}+\mathrm{PF}$ selects the user who has better average channel condition among the mobiles, then total throughput will be increased since packet data is transmitted more frequently to the user having the better average channel condition or can be transmitted with higher data rate when channel condition is proportionally better. Here, a additional parameter $\alpha$ is introduced to adjust the window size for the average requested rate. The scheduler at base station $j$ in slot $n$ for the proportional fair scheme is given as:

$$
X_{j}(n)=\left[\frac{R_{i}^{r e q}(n) \times R_{i}^{r e q *}(n)}{R_{i}^{*}(n)}\right],
$$


where $R_{i}^{r e q *}(n)$ is the average requested rate by the mobile $i$ over some time interval in slot $n$ which can be expressed as:

$$
R_{i}^{r e q *}(n+1)=\left(1-\frac{1}{\alpha T}\right) \times R_{i}^{r e q *}(n)+\frac{1}{\alpha T} \times R_{i}^{r e q}(n)
$$

where $\alpha$ is positive real number in $\left[\frac{1}{T}, 1\right] . R_{i}^{r e q}(n)$ is the requested transmission rate for user $i$ in slot $n$, and the update for the achieved average rate remains the same as in (3.9). In the above equation, the value of $\alpha T$ adjusts the window size for the averaged requested rate $R_{i}^{r e q}(n)$. If $\alpha=1$, the window size for $R_{i}^{\text {req* }}(n+1)$ equals to the window size for average rate $R_{i}^{*}(n+1)$. If $\alpha=\frac{1}{T}, R_{i}^{r e q *}(n+1)$ equal to average rate $R_{i}^{*}(n+1)$; it means that the window size for $R_{i}^{*}(n+1)$ is equal to one slot and that the scheduler gives the priority to the user having a better condition currently. The user with the highest scheduler value out of all $M$ users will receive permission for transmission at each slot. The scheduler updates the average transmission rate $R_{i}^{*}(n)$ and the average requested rate $R_{i}^{\text {req*}}(n)$ in each time slot for each mobile.

CBO scheme considers solely the channel condition to achieve higher throughput but neglects the fairness, whereas RR and RS scheme provide a high degree of fairness but suffered from the system efficiency. $\mathrm{CB}+\mathrm{PF}$ and its variant attempted to rectify the problem by the combined consideration of channel conditions and acheived throughput. However, they suffer from the fact that

1. the maximum achieved throughput at any time is not controlled, as a result, at any instant, there exists a lager variation among individual acheived rates.

2. the mapping between channel condition and the requested rate is discrete with large step (see Table 3.2), as a result, only evident variation of channel condition affects the scheduling operation.

3. the required multirate traffice is not considered.

4. it is not flexible to adjust in different network traffic control. 
We propose a scheme that takes into account the real-time channel conditions and acheived average rates while keeping the maximum acheived rate under control. A systemdependent tolerance factor is introduced. That is flexible in adjusting the maximum allowable achieved rate over the required rate.

Reproduced with permission of the copyright owner. Further reproduction prohibited without permission. 


\section{Chapter 4}

\section{Proposed Fair Scheduling Scheme $(\mathrm{CB}+\mathrm{MS}+\mathrm{FS})$}

Providing fair share of system throughput among the different data users is one of the key issues in provisioning quality of service (QoS) in wireless data networks. In this chapter, we propose a scheduling scheme that attempts to provide fair data throughput and high system average throughput based on the channel condition, achieved average rate and required rate with the assistance of individual mobile terminal. This scheme is called as CB+MA+FS since it is channel-based, mobile-assisted and fairly-shared scheduling scheme.

The fairness is achieved by using a scheduling algorithm to allocate radio resources among the mobile terminals in proportion to the combined consideration of real time channel condition, required transmission rate and achieved average rate in the proposed scheduling scheme.

\subsection{Fair Scheduling Problem}

\subsubsection{Definition of Fairness}

Let $X$ be a random variable. Then the standard deviation of $X$ can be expressed as:

$$
S D(X)=\sqrt{E\left([X-E(X)]^{2}\right)},
$$

where $E(X)$ is the mean value of random variable $X$. 
Mathematically, standard deviation reveals the deviation degree of $X$ 's value with respect to $X$ 's mean value. If most $X^{\prime} s$ values are close (or concentrated) to the mean value, then the value of standard variance of $X$ will be small, and vice versa, that is if $X^{\prime} s$ values are dispersed compared to the mean value, then the value of standard deviation of $X$ will be large. Therefore, standard deviation of $X$ can evaluate the distribution degree of $X$.

In our proposed fair scheduling scheme, we define degree of fairness $F_{k}$ as the reciprocal of standard deviation of individual throughput times $J$ in scheduling scheme $k$ which is expressed in (4.3).

$$
F_{k}=\frac{J}{S D\left(R_{i k}^{*}\right)}
$$

where

$$
\begin{aligned}
S D\left(R_{i k}^{*}\right) & =\sqrt{\frac{\sum_{i=1}^{M}\left(E\left(R_{i k}^{*}\right)-R_{i k}^{*}\right)^{2}}{M}} \\
J & =\min \left[S D\left(R_{i k}^{*}\right)\right]
\end{aligned}
$$

where $J$ is the minimum value of standard deviations of individual throughput in different scheduling schemes, $k$ denote different scheduling schemes, $k \in\{C B O, R R, R S, C B+M A+F S\}$, $R_{i k}^{*}$ is the average throughput achieved by user $i$ in scheduling scheme $k, E\left(R_{i k}^{*}\right)$ is the mean of individual throughput achieved in scheduling scheme $k$, and $M$ is total number of users.

The value of standard deviation will show how the individual mobile terminal's throughput is distributed during the scheduling period. That is the larger (smaller) the standard deviation, the lower (higher) degree of fairness the scheme $k$. We can also find out if system provides fair distribution of individual throughput to individual user (or if most mobile terminals will never be scheduled) through analysis.

The individual throughput of user $i$ can be expressed as:

$$
R_{i k}^{*}=\frac{\sum_{n=1}^{N} R_{i k}(n)}{N}
$$

where $N$ is total slot number, $R_{i k}(n)$ is the transmission rate of user $i$ in slot $n$.

We assume $R_{i k}(n)=0$ if user $i$ is not scheduled at slot $n$ in scheduling scheme $k$. Therefore, the mean of individual throughput is defined as,

$$
E\left(R_{i k}^{*}\right)=\frac{\sum_{i=1}^{M} R_{i k}^{*}}{M}
$$




$$
=\frac{\sum_{i=1}^{M} \sum_{n=1}^{N} R_{i k}(n)}{M \times N}
$$

\subsubsection{Motivation}

CBO provides maximum system throughput at the expense of fairness, because all resources can be allocated to a single user with the best channel condition and the other users have to be blocked (or delayed). However, the price paid for the high system throughput is potentially evident (as seen in Section 5) in large variations of individual arerage throughput among the mobile terminal population. The round robin scheduler cycles through the list of active users and thus is fair in sharing resources. However, as it does not take the channel quality into account it is suffering in both system throughput loss and the deprived signal quality. Therefore, in general, there is a trade-off between the fairness and the system throughput in designing a scheduler. Hence, scheduling algorithms should preferably take channel quality into account and at the same time, introduce some degree of fairness.

\subsection{Proposed Algorithm}

We propose a simple scheduling scheme that takes into account the channel conditions with the assistance of mobile terminals for fair sharing of transmission throughput. Hence we call it channel-based mobile-assisted fairly-shared $(C B+M A+F S)$ scheduling scheme.

The major consideration for any packet scheduling scheme in wireless networks is to delay or block the mobile terminals that costs the most system resources so that the system resource can be released to serve other mobile terminals with better link conditions. We also follow the same philosophy in our approach. Hence, the $C B+M A+F S$ scheme is applicable for non-real time applications.

The CB+MA+FS scheduler updates the achieved average rate at each time slot for each mobile terminal, then computes the priority factor which is a new parameter introduced in our proposed scheduling scheme for each mobile terminal based on the achieved average rate; finally, it selects the mobile terminal to be scheduled which has the maximum value of the 
scheduler for the next slot. In our scheme, the priority factor is adjusted in each slot on the basis of the previous achieved average rate.

\subsubsection{Priority Factor}

In order to take the related parameters of multirate services into account in the scheduling, a new parameter $h_{i}$, denoted as priority factor, is introduced in our proposed scheduling scheme which will affect the scheduling indicator and help the scheduler to make the decision as to which mobile terminal will be scheduled at next slot.

The main idea here is that each mobile terminal has an adjustable priority factor at each slot depending on its achieved average rate so far and required transmission rate. Our strategy is based on the combined adaptive tradeoff between priority factor $h_{i}(n)$. and achieved average rate $R_{i}^{*}(n-1)$ in specific time slot $n$ for user $i$. Therefore, we use a linear mathematical model between priority factor $h_{i}$ and achieved average rate $R_{i}^{*}$ as illustrated in Fig. 4.1. A mathematical relationship among priority factor, required rate and achieved

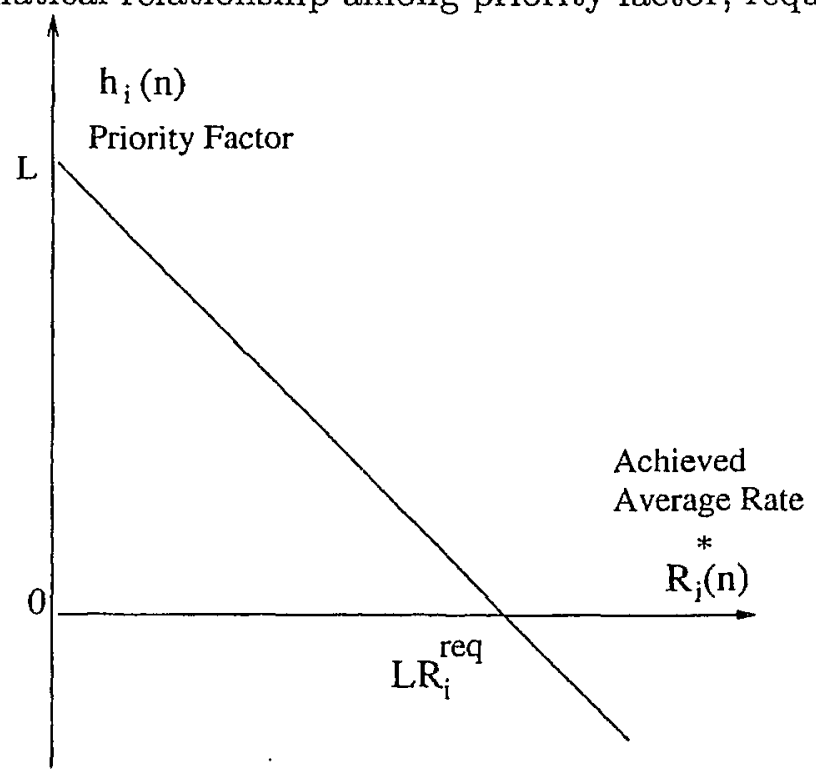

Figure 4.1: Combined Adaptive Tradeoff between Priority Factor and Achieved Average Rate. 
average rate is given as,

$$
h_{i}(n)=L-\frac{R_{i}^{*}(n-1)}{R_{i}^{r e q}}, \quad 1 \leq i \leq M, 1 \leq n \leq N
$$

where $M$ is total user number, $N$ is total slot number, $L$ is a system-dependent tolerance factor which controls the extent of the allowable maximum achieved average rate in relation to the required rate. More discussion will be found later in Section 4.3.3.

For the convenience of analysis, we normalize $h_{i}(n)$ to $H_{i}(n)$ by dividing $L$. So, we get a modified figure in Fig. 4.2 for the combined adaptive tradeoff between priority factor and achieved average rate, and a linear mathematical relationship among these parameters is given in (4.8).

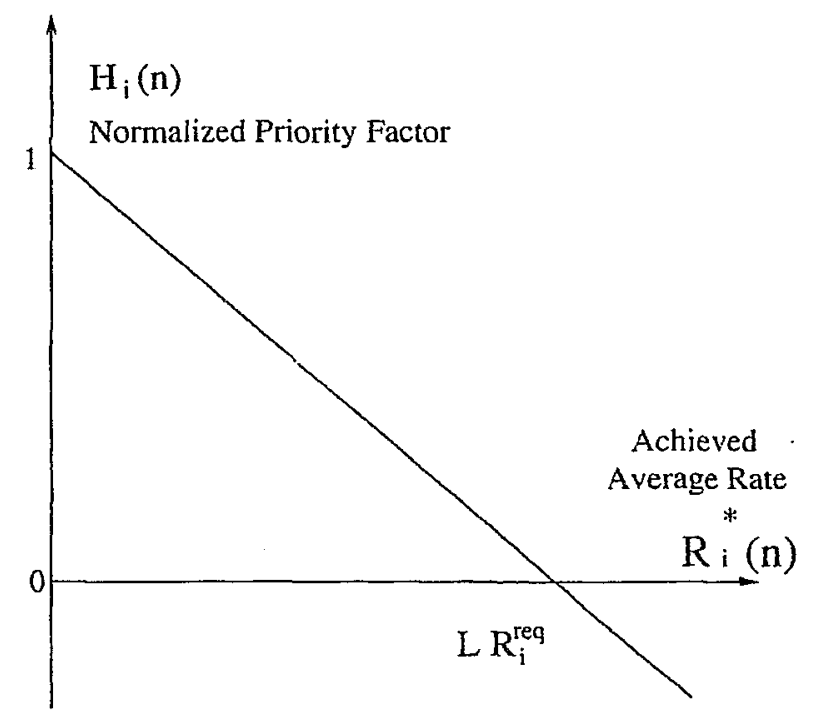

Figure 4.2: Combined Adaptive Tradeoff between Normalized Priority Factor and Achieved Average Rate.

$$
H_{i}(n)=1-\frac{R_{i}^{*}(n-1)}{L \times R_{i}^{r e q}}, \quad-1 \leq i \leq M, 1 \leq n \leq N .
$$

The strategy described using Fig. 4.2 is operated in the following way:

1. If the achieved average rate $R_{i}^{*}(n-1)$ for user $i$ is increased (decreased) at specific time slot $n-1$, then the priority factor $H_{i}(n)$ at the next slot $n$ will be decreased (increased).

Reproduced with permission of the copyright owner. Further reproduction prohibited without permission. 
2. The priority factor $H_{i}(n)$ is adjusted in the range $[-\infty, 1]$ at each slot; the achieved average rate $R_{i}^{*}(n)$ is changed in the flexible range. When achieved average rate equals $L R_{i}^{r e q}, H_{i}(n)=0$; when achieved average rate equals zero, $H_{i}(n)=1$; when achieved average rate equals $R_{i}^{\text {req }}$, then the priority factor $H_{i}(n)$ at the next slot equals $\left(1-\frac{1}{L}\right)$; when achieved average rate is greater than $L R_{i}^{r e q}$, then $H_{i}(n)<0$ at specific instant. It can be seen that the $L$ is a tolerant factor at various achieved throughput levels.

In our proposed scheduling model, the base station adjusts the priority factor of each mobile terminal depending on the achieved average rate $R_{i}^{*}(n)$ in a given slot. The higher the achieved throughput in relation to the required rate, the smaller the priority factor. It means that the mobile terminal which has the high achieved average rate in given slot will get low priority in the next slot.

\section{A Numerical Example}

We give an example to show how the combined tradeoff between priority factor and achieved average rate works. Assuming that we have two users waiting to be scheduled with the same channel quality, with the system-dependent parameter of $L=2$, and their required transmission rate of $R_{1}^{r e q}$ and $R_{2}^{r e q}$ respectively. We assume in slot 1 , user 1 gets achieved average rate $R_{1}^{*}(1)=R_{1}^{\text {req }}$, user 2 gets achieved average rate $R_{2}^{*}(1)=0.5 R_{2}^{\text {req }}$, so we can calculate the priority factor for slot 2 for both users as,

$$
\begin{gathered}
H_{1}(2)=1-\frac{R_{1}^{*}(1)}{L \times R_{1}^{r e q}}=1-0.5=0.5 \\
H_{2}(2)=1-\frac{R_{2}^{*}(1)}{L \times R_{2}^{r e q}}=1-0.25=0.75
\end{gathered}
$$

Since $H_{1}(2)<H_{2}(2)$, user 2 is scheduled to be transmitted in slot 2 while user 1 is blocked. Therefore, we can find that priority factor controls the fair throughput allocation effectively based on the achieved average rate in multirate CDMA cellular systems. 


\subsubsection{Achieved Average Rate}

Achieved average rate is a very important parameter in our proposed scheduling scheme since the scheduler will calculate it in each slot, and priority factor will be adjusted based on the achieved average rate.

Next we introduce two methods to update achieved average rate.

\section{Method 1:}

To be specific, assume that there are total of $M$ users, total number of slot is $N$, and during each time slot the current transmitting rate is fixed. The forward link of the proposed cellular CDMA system consists of multirate service channel which is divided into $1.67 \mathrm{~ms}$ time slots. The average throughput received by the mobile over a window of appropriate size $T$. This window size is the total transmission duration we assumed before the updating slot is coming. This method is shown in Fig. 4.3. From the figure, we can update the average

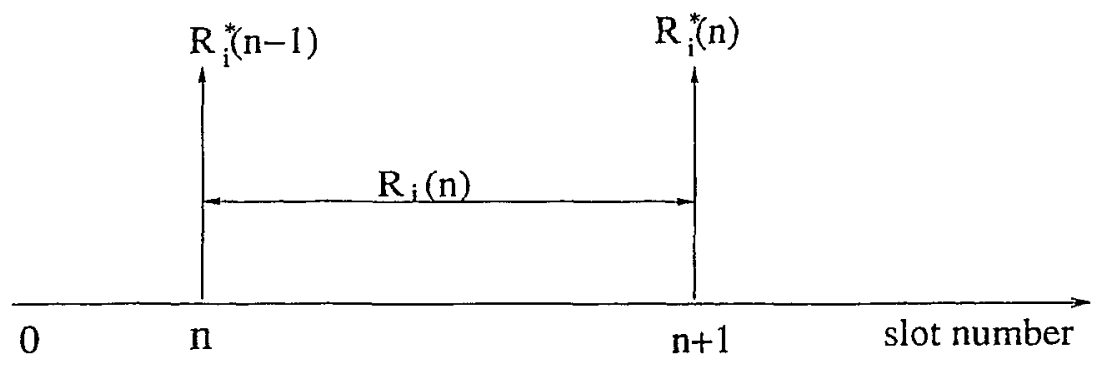

$T$ ( update window size)

Figure 4.3: Time Slot Structure of Method 1 for Updating Achieved Average Rate

rate at $n^{\text {th }}$ time slot for user $i$ as:

$$
T \times R_{i}^{*}(n)=(T-1) R_{i}^{*}(n-1)+R_{i}(n) \times 1
$$


The equation (4.9) is divided by $T$ in both sides to get the achieved average rate,

$$
R_{i}^{*}(n)=(1-1 / T) R_{i}^{*}(n-1)+1 / T \times R_{i}(n)
$$

\section{Method 2:}

Now, we introduce the second method to update the achieved average rate and it is graphically shown in Fig. 4.4. We can update the average rate at $(n+1)^{\text {th }}$ time slot for user $i$

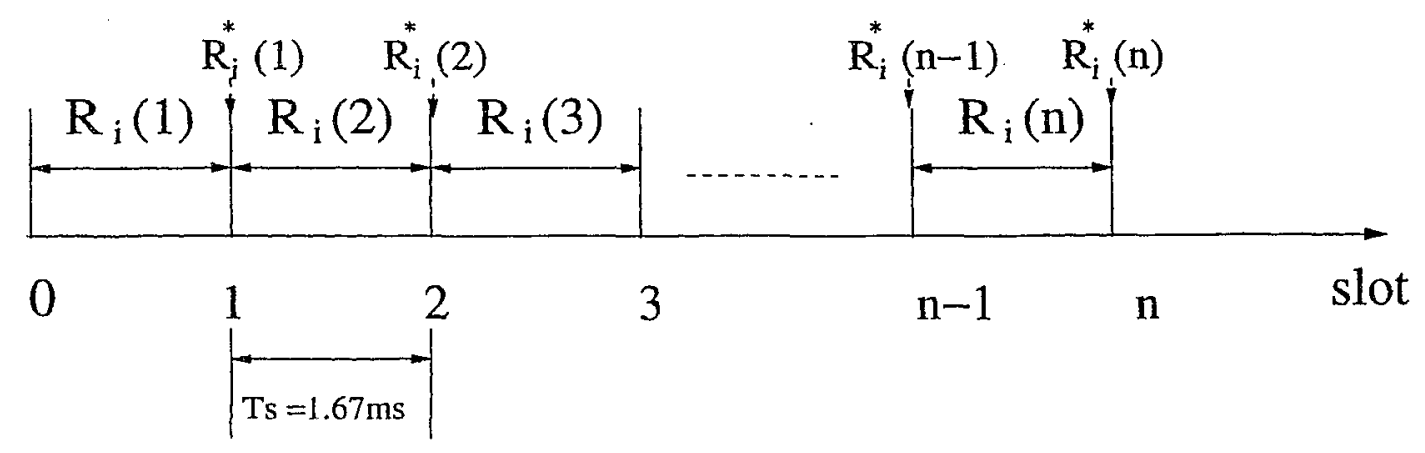

Figure 4.4: Time Slot Structure of Method 2 for Updating Achieved Average Rate

as:

$$
\begin{aligned}
R_{i}^{*}(n+1) & =\frac{R_{i}(1) \times T_{s}+R_{i}(2) \times T_{s}+\ldots .+R_{i}(n) \times T_{s}}{N \times T_{s}} \\
& =\frac{\sum_{n=1}^{N} R_{i}(n)}{N}
\end{aligned}
$$

where $R_{i}(n)$ is the transmission rate of user $i$ at slot $n, T_{s}$ is the length of each slot.

We use the method 2 in our scheme which is applicable to fixed-slot size system.

\subsubsection{Scheduling Indicator}

In the proposed scheduling scheme, the scheduling indicator in the base station determines the next terminal to be served based on priority factor and channel condition. When there are multiple users requesting services from scarce system resources, the scheduling indicators determine the user that will be served. Mobile terminal $i$ gets assigned priority factor $H_{i}(n)$ 
in slot $n$ depending on its achieved average rate $R_{i}^{*}(n)$ and required transimission rate $R_{i}^{\text {req }}$. Now, we introduce the scheduling indicator $Z_{i j}(n)$ of user $i$ in slot $n$ at base station $j$ as :

$$
Z_{i j}(n)=\left[H_{i}(n) \times G_{i j}(n)\right], \quad 0<n \leq N, 0<i \leq M,(n, i) \in I
$$

where $G_{i j}(n)$ is the link gain between mobile terminal $i$ and its home base station $j$. Note that:

$$
H_{i}(n)=1-\frac{R_{i}^{*}(n)}{L \times R_{i}^{r e q}}
$$

The scheduler at the base station computes $Z_{i j}(n)$ for each user in each slot and selects the mobile terminal to be transmitted that has the larger scheduling indicator value. The base station ranks all users based on the above indicator. The scheduling indicator is activated to delay (or block) the users with small indicator value. In our scheme, it is assumed that base stations have access to all the parameters required to determine the scheduling indicator $Z_{i j}(n)$ except for forward link channel gain $G_{i j}(n)$. It is expected that the mobiles will assist (perhaps via reverse link pilot power) in this regard.

\subsection{Analysis}

\subsubsection{System Throughput}

The performance of the proposed scheduling scheme is analyzed in terms of throughput. System throughput is a very important parameter to evaluate the system performance among different scheduling schemes. System throughput $T_{k}$ is defined as the sum of individual throughput which can be expressed as:

$$
\begin{aligned}
T_{k} & =\sum_{i=1}^{M} R_{i k}^{*} \\
& =\frac{\sum_{i=1}^{M} \sum_{n=1}^{N} R_{i k}(n)}{N}
\end{aligned}
$$

where $k \in\{\mathrm{CBO}, \mathrm{RR}, \mathrm{RS}, \mathrm{CB}+\mathrm{MA}+\mathrm{FS}\}$. 
Since mean of individual through ut $E\left(R_{i k}^{*}\right)$ is already defined in (4.6), we can see that

$$
T_{k}=E\left(R_{i k}^{*}\right) \times M
$$

As evident in (4.33), the system throughput is related to the current transmission rate of individual mobile terminal in each time slot. Hence, the current transmission rate will be discussed next.

We know that in the downlink of a cellular CDMA system, the expression $E_{b} / N_{0}$ of mobile terminal $i$ in slot $n$ can be given by $\gamma_{i}$ under the assumption of home base station $j$ as,

$$
\gamma_{i}(n)=\frac{W}{R_{i}(n)} \frac{P_{i} G_{i j}(n)}{\left(P-P_{i}\right) G_{i j}(n)+\sum_{m \neq j} P G_{i m}(n)},
$$

where $W$ is the bandwidth, $P$ is the total transmission power from base station, $P_{i}$ is the transmitted power for user $i, G_{i j}(n)$ is the link gain between user $i$ and home base station $j$ in slot $n, m$ is the neighboring base station.

Here, we assume the each base station schedules only one mobile terminal in each slot and all base stations have the same transmission power, hence, $\gamma_{i}$ can be simplified to the expression as below:

$$
\gamma_{i}(n)=\frac{W}{R_{i}(n)} \frac{G_{i j}(n)}{\sum_{m \neq j} G_{i m}(n)} .
$$

We further assume that service quality $\gamma_{i}$ for multirate users are equal for all users during the scheduling duration ( $i . e, \gamma_{i}=\gamma, \forall i$ ). Then, the current transmission rate for user $i$ in slot $n$ can be derived as,

$$
R_{i}(n)=\frac{W}{\gamma} \frac{G_{i j}(n)}{\sum_{m \neq j} G_{i m}(n)}
$$

Since $W / \gamma$ is a constant, the expression of $R_{i}(n)$ can be simplified to:

$$
R_{i}(n)=C \times \frac{G_{i j}(n)}{\sum_{m \neq j} G_{i m}(n)} .
$$

where $C=W / \gamma$. By substituting (4.19) in (4.14), the system throughput $T_{k}$ can be rewritten as:

$$
T_{k}=\frac{C}{N} \sum_{i=1}^{M} \sum_{n=1}^{N} \frac{G_{i j}(n)}{\sum_{m \neq j} G_{i m}(n)}
$$


We note that $T_{k}$ is related to the quotient of the real-time link gain of the scheduled mobile terminal and the total interference from other link gains in each slot.

In $\mathrm{CBO}$, the mobile terminal which has the best link gain will be scheduled in each slot, and the total interference which comes from the neighboring base station is much smaller

So, we can expect that

$$
\frac{G_{i j}^{C B O}(n)}{\sum_{m \neq j} G_{i m}^{C B O}(n)} \gg 1, \quad \forall n, i .
$$

In our proposed $\mathrm{CB}+\mathrm{MA}+\mathrm{FS}$ scheme, the link gain is not the only factor used to decide which mobile terminal will be scheduled. We consider the combined effect of priority and channel condition together. The mobile terminal which has the best channel conditon can not be scheduled always. Because, along with the increase in the achieved average throughput of individual user, the priority value will be decreased; so the mobile terminal which has the maximum link gain will be blocked at specific slot after transmitting for some time. Then, the other user who has relatively large product of priority value and link gain will be scheduled in the next slots. Therefore, in each slot, the mobile terminal which has higher priority and relatively better channel condition (maybe the best, maybe not the best) will be scheduled. So, we can expect that

$$
\frac{G_{i j}^{C B+M A+F S}(n)}{\sum_{m \neq j} G_{i m}^{C B+M A+F S}(n)}>1, \quad \forall n, i .
$$

In $R R$ scheduling scheme, the scheduler cycles through the list of active mobile terminals in a fair manner and it does not consider the channel quality. It means that $R R$ schedules the mobile terminal no matter what kind of channel condition it has at a specific slot. RS scheduling scheme has the same situation as RR scheme over a large duration since its scheduler randomly selects the users to be transmitted. In both RS and RR cases, the link gain of the scheduled user in each slot may be good or bad. Although we do not know the exact channel condition in each slot, but if we select a very large scheduling slot duration, we can expect that the quality of channel conditon of the scheduled mobile terminal in each slot is uniformly distributed. It means that the probabilities of good and bad channel conditions 
occuring during scheduling duration are the same since the mobile terminals are uniformly distributed. RR and RS will have the same situation that, in half of the scheduling slots,

$$
\frac{G_{i j}^{R R, R S}(n)}{\sum_{m \neq j} G_{i m}^{R R, R S}(n)}>1, \quad \forall n, i
$$

and in the other half of the scheduling slots,

$$
\frac{G_{i j}^{R R, R S}(n)}{\sum_{m \neq j} G_{i m}^{R R, R S}(n)}<1, \quad \forall n, i
$$

Since the system throughput is the sum of the quotient of the real-time link gain of the scheduled user and its interference link gains in each slot as seen in (4.20), and we have the following relationship:

$$
\frac{G_{i j}^{C B O}(n)}{\sum_{m \neq j} G_{i m}^{C B O}(n)}>\frac{G_{i j}^{C B+M A+F S}(n)}{\sum_{m \neq j} G_{i m}^{C B+M A+F^{S} S}(n)}>\frac{G_{i j}^{R R, R S}(n)}{\sum_{m \neq j} G_{i m}^{R R, R S}(n)} .
$$

From the above relationship, we can conclude that $\mathrm{CBO}$ has the highest system average throughput, $\mathrm{CB}+\mathrm{MA}+\mathrm{FS}$ has good system average throughput, and the system average throughput of $R R$ and $R S$ is worse than $C B O$ and $C B+M A+F S$. That is,

$$
T_{C B O}>T_{C B+M A+F S}>T_{R R, R S}
$$

In the above we have done an analysis based on the relative channel gain between the scheduled user and the other interference links. To validate our claim, we did a simulation study (in Section 5) and simulation results also confirm our conclusion.

\subsubsection{Standard Deviation of Individual Throughput}

The standard deviation of individual throughput which is defined in Section 4.1 .1 can be expressed as:

$$
S D\left(R_{i k}^{*}\right)=\sqrt{\frac{\sum_{i=1}^{M}\left(E\left(R_{i k}^{*}\right)-R_{i k}^{*}\right)^{2}}{M}}
$$

Note that $R_{i k}^{*}$ is the achieved average rate of user $i$ in scheduling scheme $k, E\left(R_{i k}^{*}\right)$ is mean of individual throughput. For the convenience of analysis, we introduce $B_{k}$ which is the 
sum of the square of the difference between mean of individual throughput and individual throughput in scheduling scheme $k$. So, the standard deviation can be expressed as,

$$
S D\left(R_{i k}^{*}\right)=\sqrt{\frac{B_{k}}{M}},
$$

where

$$
B_{k}=\sum_{i=1}^{M}\left(E\left(R_{i k}^{*}\right)-R_{i k}^{*}\right)^{2} .
$$

We can observe that standard deviation of individual throughput is the square root of the quotient of $B_{k}$ and $M$ ( $M$ is a constant). Therefore, for the convenience of analysis, we analyze the characteristic of $B_{k}$ which can be extended to,

$$
\begin{aligned}
B_{k} & =\left(E\left(R_{i k}^{*}\right)-R_{1 k}^{*}\right)^{2}+\left(E\left(R_{i k}^{*}\right)-R_{2 k}^{*}\right)^{2}+\ldots . .+\left(E\left(R_{i k}^{*}\right)-R_{M k}^{*}\right)^{2} \\
& =E\left(R_{i k}^{*}\right)^{2}-2 E\left(R_{i k}^{*}\right) R_{1 k}^{*}+\left(R_{1 k}^{*}\right)^{2}+\ldots . .+E\left(R_{i k}^{*}\right)^{2}-2 E\left(R_{i k}^{*}\right) R_{M k}^{*}+\left(R_{M k}^{*}\right)^{2} \\
& =M \times E\left(R_{i k}^{*}\right)^{2}-2 E\left(R_{i k}^{*}\right)\left(R_{1 k}^{*}+R_{2 k}^{*}+\ldots .+R_{M k}^{*}\right)+\left(R_{1 k}^{*}\right)^{2}+\left(R_{2 k}^{*}\right)^{2}+\ldots . .+\left(R_{M k}^{*}\right)^{2} \\
& =M \times E\left(R_{i k}^{*}\right)^{2}-2 M \times E\left(R_{i k}^{*}\right)^{2}+\left(R_{1 k}^{*}\right)^{2}+\left(R_{2 k}^{*}\right)^{2}+\ldots . .+\left(R_{M k}^{*}\right)^{2}
\end{aligned}
$$

Since

$$
\begin{aligned}
E\left(R_{i k}^{*}\right)^{2} & =\left(\frac{R_{1 k}^{*}+R_{2 k}^{*}+\ldots .+R_{M k}^{*}}{M}\right)^{2} \\
& =\frac{\left(R_{1 k}^{*}\right)^{2}+\left(R_{2 k}^{*}\right)^{2}+\ldots .+\left(R_{M k}^{*}\right)^{2}+2 \sum_{i \neq j} R_{i k}^{*} R_{j k}^{*}}{M^{2}}
\end{aligned}
$$

$B_{k}$ can be expressed as:

$$
B_{k}=M \times(M-1) \times E\left(R_{i k}^{*}\right)^{2}-2 \sum_{i \neq j} R_{i k}^{*} R_{j k}^{*} .
$$

We already know that

$$
T_{k}=E\left(R_{i k}^{*}\right) \times M,
$$

So, we get the relationship between $B_{k}$ and system throughput $T_{k}$ as,

$$
B_{k}=\left(1-\frac{1}{M}\right) \times\left(T_{k}\right)^{2}-2 \sum_{i \neq j} R_{i k}^{*} R_{j k}^{*}
$$


Since $S D\left(R_{i k}^{*}\right)$ is proportional to $B_{k}$ value, and it is increased along with the increase of $B_{k}$ value, and vice versa, we analyze the value of $B_{k}$ in four scheduling schemes. For the analysis, we assume the channel conditions are all distance-dependent only, and in each slot only one user is scheduled for one base station.

In $\mathrm{CBO}$, the user with the best channel quality is always scheduled and the other users are always blocked during the scheduling; hence,

$$
R_{i k}^{*}=0, \quad 0<i<M, i \neq s
$$

where $s$ is the index of the scheduled user. Therefore, $B_{k}$ in $C B O$ is expressed as,

$$
B_{C B O}=\left(1-\frac{1}{M}\right) \times\left(T_{C B O}\right)^{2}
$$

From the previous analysis about system throughput in section 4.3.1, we already know that

$$
T_{C B O}>T_{C B+M A+F S}>T_{R R, R S}
$$

Since $\mathrm{CBO}$ has the best system throughput $T_{C B O}$ and the reduction item $\sum_{i \neq j} R_{i k}^{*} R_{j k}^{*}$ $=0$, we can expect that CBO has maximum standard variance of individual throughput among these four scheduling schemes .

In $\mathrm{CB}+\mathrm{MA}+\mathrm{FS}$ and RS schemes, some users are scheduled, the others are not. It means that some users get $R_{i k}^{*}=0$. We will get some component of $\left(R_{i k}^{*} \times R_{j k}^{*}\right)$ equal to 0 . And the sum of these products will be smaller than RR. Since CB+MA+FS has higher system throughput than $R R$ and $R S$, and the reduction item in the variance of throughput in $\mathrm{CB}+\mathrm{MA}+\mathrm{FS}$ is smaller than $\mathrm{RR}, \mathrm{CB}+\mathrm{MA}+\mathrm{FS}$ has larger standard variance of individual throughput than $\mathrm{RR}$ and $\mathrm{RS}$.

Comparing with $\mathrm{RR}$ and $\mathrm{RS}$, if the scheduling duration is short, then the reduction item in variance of $R S$ is smaller than $R R$, so $R S$ will have larger standard deviation than $R R$. But if the scheduling duration is long enough, then RS and RR will have same scheduling situation, so the standard deviation of individual throughput in RS and RR is almost equal.

Finally, we can conclude that $\mathrm{CBO}$ has the maximum standard deviation of individual throughput, $\mathrm{CB}+\mathrm{MA}+\mathrm{FS}$ has relatively large standard deviation of individual throughput, 
the standard deviation of individual throughput in RR and RS is smaller than $\mathrm{CBO}$ and $\mathrm{CB}+\mathrm{MA}+\mathrm{FS}$. That is,

$$
S D\left(R_{i, C B O}^{*}\right)>S D\left(R_{i, C B+M A+F S}^{*}\right)>S D\left(R_{i,(R R, R S)}^{*}\right)
$$

Since fairness is defined as $F_{k}$, which can be expressed as

$$
F_{K}=\frac{J}{S D\left(R_{i, k}^{*}\right)}
$$

where $J=\min \left[S D\left(R_{i, k}^{*}\right)\right]$, therefore,

$$
F_{C B O}>F_{C B+M A+F S}>F_{R R, R S}
$$

Simulation results confirm this analytical observation.

\subsubsection{System Tolerance Factor - L}

In our linear priority control model, a parameter $L$ was introduced (see Fig. 4.2). It is system-dependent and determines how tolerant the system is with respect to the achieved average rate in relation to required rate. $L$ controls not only the maximum range of average throughput being achieved from multirate users at any time instant but also impacts the priority factor in the adjustment in each slot. System average throughput is also affected finally by $L$, since the maximum average throughput achieved by the individual user is decided by $L$. Let us analyze how the value of $L$ determines the maximum achieved average throughput of each user.

System dependent parameter $L$ is an positive constant ( $L>0$ ). From Fig. 4.2, we find that the intersection point in the $\mathrm{X}$ axis is the maximum average rate achieved during a long time by each mobile terminal $i$ in slot $n$ which equals to the product of $L$ and individual required rate. That is,

$$
\max \left[R_{i}^{*} .(n)\right]=L \times R_{i}^{r e q}, \quad 0<i<M, 0<n<N,(i, n) \in I
$$

It means that the average throughput achieved by individual user so far in each slot can not be larger than the maximum value since along with the increase of achieved average rate 
of the scheduled mobile terminal, the priority factor value will be decreased, when average throughput achieves its maximum value, the priority equals 0 (see (4.8)). In fact, before the average throughput achieves its allowable maximum value, priority factor has already been decreased to a smaller value. Therefore, the scheduled mobile terminal $i$ will be blocked in slot $(n+1)$ because it may have relatively smaller scheduler value. But, in slot $(n+2)$, $R_{i}^{*}(n+2)$ will be decreased because of being blocked in slot $(n+1)$, then it achieves a increased priority value, and the priority value will keep increasing if user $i$ keeps blocked (or delayed) in the consecutive slots, until it is scheduled again. This is how our scheduling mechanism works.

From the above discussion, we can note that the system-dependent parameter $L$ is sensitive to the scheduling system since it decides the maximum average throughput achieved by the individual user, or in other words, it decides the longest duration of being scheduled for each mobile terminal. Because, higher maximum achieved average throughput means longer scheduling duration for the current scheduled mobile terminal. Therefore, $L$ will finally affect the fairness in our proposed scheduling scheme. We can note that,

1. The smaller the $L$ value, the fairer scheduling in terms of throughput, because $L$ limits the maximum allowable throughput.

2. If $L$ value is very small $(L \approx 0), H_{i}(n)=-\infty, \forall i$, as shown in Fig. 4.5, then the scheduling indicator $Z_{i j}(n)=-\infty, \forall i$, the scheduling scheme becomes the case of absolute fairness scheme (like RR or RS).

3. If $L$ value is very large $(L \gg 1), H_{i}(n)=1, \forall i$, as shown in Fig. 4.6 , then the scheduling indicator $Z_{i j}(n)=G_{i j}(n)$, the scheduling scheme becomes the case of absolute unfairness scheme (like CBO).

Next, we will analyze how the value of $L$ affects the step of priority factor being adjusted in each slot which is shown in Fig. 4.7. We set $L$ as two values: $L_{1}$ and $L_{2}$, let $L_{1}<L_{2}$. We 


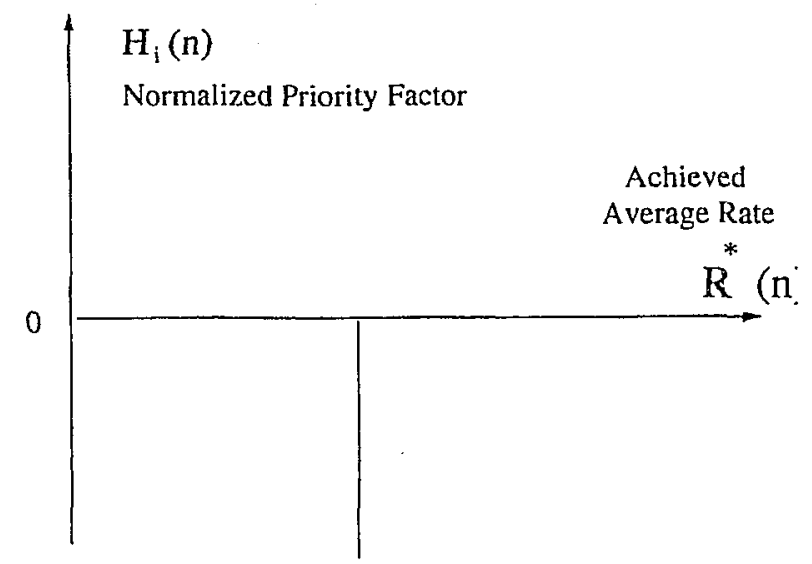

Figure 4.5: Priority Factor with $\mathrm{L}=0$.

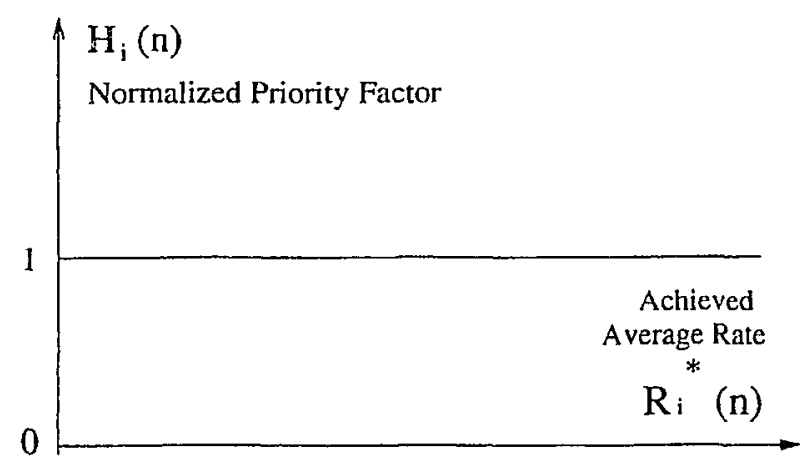

Figure 4.6: Priority Factor with $\mathrm{L}=\infty$.

Reproduced with permission of the copyright owner. Further reproduction prohibited without permission. 


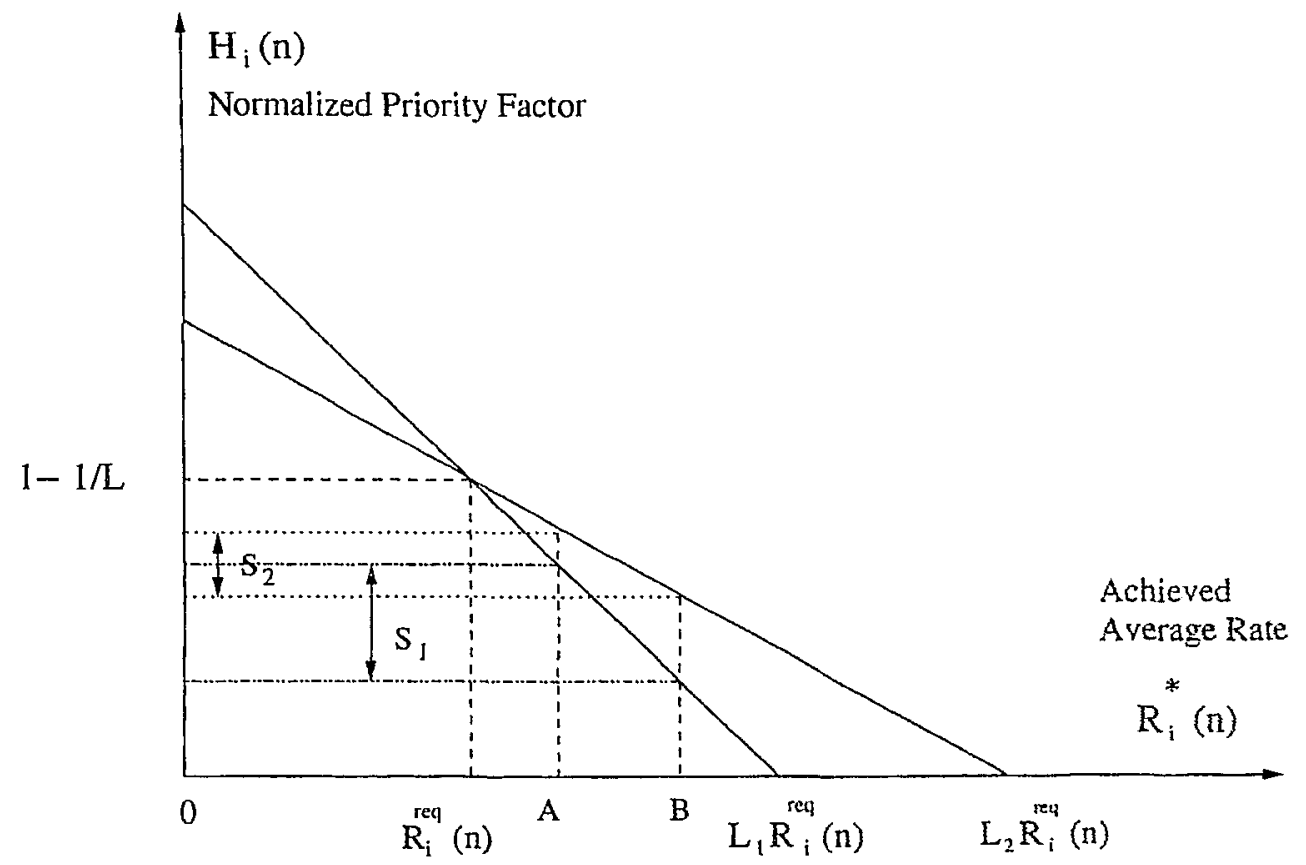

Figure 4.7: Steps of Priority Factor with different value of $L$.

assume that the achieved average rate of user $i$ is increased from $A$ (see $\mathrm{X}$ axis) in slot $n$ to $B$ point (see $Y$ axis) in slot $(n+1)$. From Fig.5.5, we can get $S_{1}$ and $S_{2}$ as the step change values of priority factor respectively for $L_{1}$ and $L_{2}$, and we find that $S_{1}>S_{2}$.

Therefore, the larger $L$ value is, the smaller step change of priority $H$ is. It means that the value of $L$ affects the adjusting speed of $H$. In each slot, $H$ will be adjusted slenderly if $L$ is set to a large value, such as $L \gg 1$, and $H$ will be adjusted evidently if $L$ is set to a small value, such as $L<1$. We know that the adjusting speed of $H$ will affect the fairness since it decides how long the individual mobile terminal will be scheduled before it achieves its maximum achieved average throughput.

Finally, we conclude that the larger (smaller) the $L$ value is, the smaller (larger) the adjusting step of $H$ in each slot is, then the more fairer and quicker the scheduling scheme is. 


\subsubsection{Signal Quality - SIR}

Signal to interference ratio - SIR is an important parameter to evaluate the system performance. In a multicell CDMA system, if it is assumed that the base station $j$ is the home base station of the mobile terminal $i$, and total base station has equal transmission power $P, S I R_{i}$ can be given as [4]:

$$
S I R_{i}=\frac{P_{i} G_{i j}}{\left(P-P_{i}\right) G_{i j}+\sum_{m \neq j} P G_{i m}}
$$

where $P_{i}$ is the transmitted power to the mobile terminal $i$ from its home base station, $G_{i j}$ is the link gain between user $i$ and its home base station $j$.

If only one user is scheduled in each slot and CDMA system is a two-cell system, SIR in slot $n$ can be expressed as:

$$
S I R_{i}(n)=\frac{P G_{i j}(n)}{\sum_{m \neq j} P G_{i m}(n)}=\frac{G_{i a}}{G_{i b}}, \quad 0<i \leq M, 0<n \leq N,
$$

where $j$ is index of base station and $j \in[a, b]$.

In $\mathrm{CBO}$, the scheduler always chooses the user with the maximum link gain in each slot, so $G_{i a} \gg G_{i b}$ or $\frac{G_{i a}}{G_{i b}} \gg 1$. Therefore, CBO will achieve best signal quality in each slot.

In $C B+M A+F S$, the scheduler selects the user based on the combined effect of channel condition and priority factor. So the channel condition of the scheduled user can not be always best and it is can not be worst too. We can expect that the channel condition being scheduled in each slot is relatively good.

In RR and RS, the scheduler chooses the mobile terminal to be scheduled with no consideration of channel quality, so it can be good or bad. We can expect that the channel of the scheduled user under consideration in each slot will be worse than $\mathrm{CB}+\mathrm{MA}+\mathrm{FS}$ and $\mathrm{CBO}$.

Finally, we can conclude that $\mathrm{CBO}$ has the best system signal quality during scheduling, $\mathrm{CB}+\mathrm{MA}+\mathrm{FS}$ has relative good system signal quality, SIRs in RR and RS are worse than in $\mathrm{CBO}$ and $\mathrm{CB}+\mathrm{MA}+\mathrm{FS}$. We have the relationship about SIR below.

$$
S I R_{C B O}>S I R_{C B M A F S}>S I R_{R R, R S}
$$




\subsection{Comparison with Existing Schemes}

In this section we do a qualitative comparison of existing schemes with the proposed scheduling scheme.

\subsubsection{Complexity}

The schedulers of different scheduling schemes work through different operations, but they are unique in that only one mobile terminal is scheduled in each slot.

In $\mathrm{CBO}$, base station computes the channel quality of each mobile terminal, then selects the mobile terminal which has the best channel condition to be scheduled, and the other mobile terminals will be blocked. Base station always schedules the user with the best channel condition and assigns the scheduled mobile terminal the current transmission rate which is proportional to real-time link gain under the condition that the signal quality is guaranteed.

In the proposed $\mathrm{CB}+\mathrm{MA}+\mathrm{FS}$, the scheduler which is expressed in (4.12) computes the channel quality of each mobile terminal in each slot with the mobile terminal assistance, then calculates the achieved average rate of each mobile terminal. Then, the scheduler computes the individual priority value. The scheduler in base station calculates the value of scheduling indicator based on link gain and priority factor, then selects the mobile terminal to be transmitted which has the maximum value of scheduling indicator. In each slot, the scheduler has to compute and record the transmission rate of each user which is used to calculate the achieved average rate of each user.

In $R R$, base station cycles through the list of active mobile terminals and does not consider the real time channel condition.

In $\mathrm{RS}$, base station randomly selects the mobile terminal to be transmitted and does not consider the real time channel condition.

From the above discussion, we know that the schedulers in RR and RS need not perform any calculation. The scheduler in CBO operates with added complexity since it computes the real-time channel condition of each user, then compares and selects the maximum value in 
each slot. From the expression of scheduler in $\mathrm{CB}+\mathrm{MA}+\mathrm{FS}$, we can see how various factors are taken into account in determining who gets scheduled in each slot. The operation of the scheduler in $\mathrm{CB}+\mathrm{MA}+\mathrm{FS}$ is most complex among these scheduling schemes since the scheduler needs to calculate and record many parameters, such as, channel condition, achieved average rate, current transmission rate and priority factor. In each slot, the scheduler has to compare the parameter value and select the user to be scheduled.

\subsubsection{Throughput}

CBO can achieve the highest system throughput since the scheduler always selects the mobile terminal with the best channel condition and assigns the highest transmission rate to the scheduled user. The system average throughput of CB+MA+FS is higher than RR and RS because the scheduler in CB+MA+FS takes the channel condition into account although it is not only the factor to decide the scheduling procedure while the schedulers in RR and RS choose the users with no consideration about channel condition (or quality of service).

\subsubsection{Fairness}

Conventional channel-based scheduling scheme neglected fairness. That is, the users with poor link quality are always blocked, and the users with good link quality are always selected to be transmitted. In our proposed $\mathrm{CB}+\mathrm{MA}+\mathrm{FS}$, under the poor link conditions, the users may get chances to transmit with high priority which is a partial fairness. RR is an absolutely fair scheduling scheme since each user gets the same chance to transmit. RS is also an fair scheduling scheme. If we set total slots duration long enough, we can expect that $R R$ and RS has same degree of fairness.

\subsubsection{Delay}

Average delay is the average waiting time of a mobile terminal to receive the next transmission packet from the base station.

In $\mathrm{CBO}$, most users are delayed for the entire scheduling duration since the mobile 


\begin{tabular}{|c|c|c|c|c|}
\hline Parameter & CBO & CB+MA+FS & RS & RR \\
\hline Location-Dependent & Yes & Yes & No & No \\
\hline Complexity & High & Higher & Low & Low \\
\hline Fairness & No & Good & Better & Best \\
\hline System Throughput & Best & Good & Worse & Worse \\
\hline Signal Quality & Best & Good & Worse & Worse \\
\hline Average Delay & Worse & Good & Good & Best \\
\hline
\end{tabular}

Table 4.1: Performance Comparison of Different Scheduling Schemes.

terminal with the best channel condition will transmit always, so CBO has the longest average delay for the most mobile terminals. RR has the same average delay situation to all the mobile terminals since each user has the same waiting time. In RS and CB+MA+FS, the delay situation is better than $\mathrm{CBO}$, but worse than $\mathrm{RR}$ since a portion of users can transmit, the others have to wait.

Table 4.1 summarizes the performance comparison for different scheduling schemes. 


\section{Chapter 5}

\section{Simulation and Results}

We simulated four scheduling algorithms ( $C B O, R R, R S, C B+M A+F S$ ) to evaluate their performance.

\subsection{Simulation Model}

\subsubsection{Simulation Setup}

The system being considered is assumed to be a cellular CDMA system which includes two cells with time division multiplexing in the forward link. The cellular system model structure is illustrated in Figure 5.1.

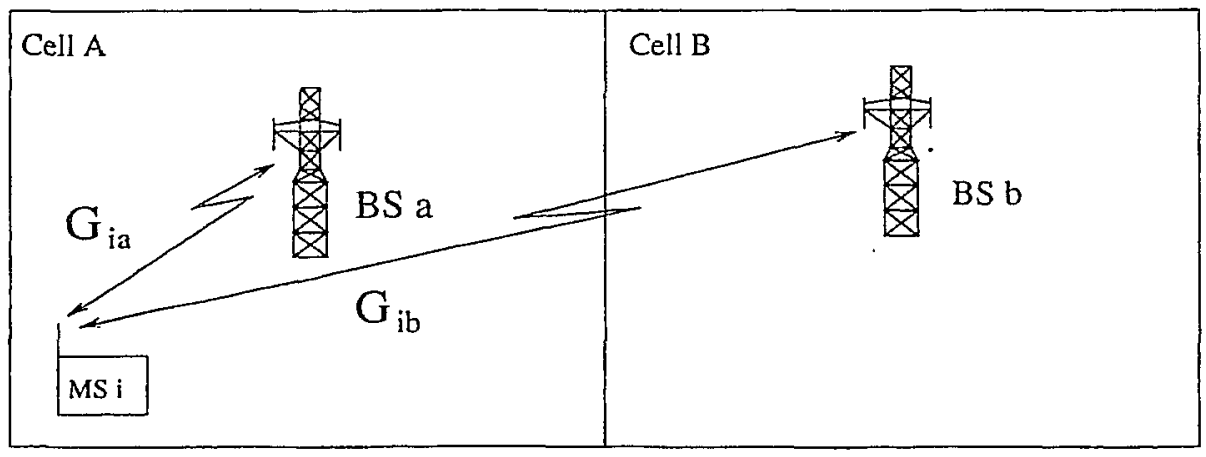

Figure 5.1: A Cellular CDMA System with Two Cells. 
We assume that the wireless network includes $M \times 2=200$ users (each cell have 100 users) that are uniformly distributed in two-cell service areas separately. The schedulers in two cells schedule the mobile terminals separately. The following conditions are assumed:

1. Mobile terminals and base stations use single omnidirectional antenna for transmission and reception. Transmitters are assumed to be capable of transmitting multirate in each slot.

2. The radius of each cell is 500 meters and the maximum distance between mobile terminal and its home base station is 700 meters. The base station is located at the center of each cell.

3. The chip rate is fixed and the total bandwidth $W(\mathrm{MHz})$ is used by all users. For all users, by fixing the chip rate, all baseband data are spread across the entire available bandwidth.

4. The transmission power allocated to the scheduled mobile terminal in each slot is full power allocation from its home base station. And both base stations have equal amount of power to be used.

5. The medium access interference is the dominant interference source in our large capacity cellular system; hence, thermal noise is neglected. The main interference in our simulation comes from the base station located in neighboring cell.

6. The scheduler in base station schedules only one mobile terminal in each slot, which results in zero intra-cell interference.

7. Perfect slot synchronization is assumed. The spread sequences are asynchronous among the arriving packets at the receiver.

8. The data rate supported on the forward link in the simulation varies from $100 \mathrm{kbps}$ to $800 \mathrm{kbps}$. The signal quality requirement is assumed to be the same for multiraie users $\left(\gamma_{i}^{r e q}=\gamma=10 \mathrm{~dB}, \forall i\right)$. 


\begin{tabular}{|c|c|c|}
\hline & Parameter & Value \\
\hline 1 & Cell Layout & 2 cells \\
\hline 2 & BS Transmit Power & $5 \mathrm{~W}$ \\
\hline 3 & BS Number & 2 \\
\hline 4 & Cell Radius & $500 \mathrm{~m}$ \\
\hline 5 & Distribution of MS & Uniform \\
\hline 6 & Fading Model & Rayleigh fading \\
\hline 7 & Propagation Coefficient & $\alpha=2$ \\
\hline 8 & Bandwidth & W $=1.25 \mathrm{MHz}$ \\
\hline 9 & Signal Quality Requirement & $\gamma=10 \mathrm{~dB}$ \\
\hline 10 & Multirate & $100 \mathrm{kbps} \sim 800 \mathrm{kbps}$ \\
\hline 11 & Total Number of Slots & 500 \\
\hline 12 & Length of Each Slot & $T_{s}=1.67 \mathrm{~ms}$ \\
\hline 13 & Total Number of Users in a Cell & 100 \\
\hline 14 & System Tolerance Factor & $\mathrm{L}=0.5$ \\
\hline
\end{tabular}

Table 5.1: Simulation Parameters.

9. We assume that for each run, the individual mobile terminal is stationary; however the channel condition of each user may be varied in different slots due to multipath fading (when it is considered).

10. Information is collected from each traffic source during fixed-length time-slots, each with a period of $T_{s}=1.67 \mathrm{~ms}$.

11. Soft handoff is not considered.

12. Evaluation is done separately for two cases: (1) with only distance-dependent path loss and (2) with distance-dependent path loss and multipath fading (modeled as Rayleigh fading).

Simulation parameters are shown in Table 5.1. 


\subsubsection{Simulation Process}

We simulated Channel-Based Only scheduling scheme (CBO), Round Robin scheduling scheme (RR), Random scheduling scheme (RS), and Channel-Based Mobile-Assisted FairlyShared scheduling scheme (CB+MA+FS). In the simulation, we assume each user will have the same required signal quality although each has different data traffic flows. We assume the initial transmission rate of mobile terminal $i$ in slot 0 equals individual required transmission rate, and the initial achieved average rate of mobile terminal $i$ in slot 0 equals zero. That is,

$$
\begin{gathered}
\gamma_{i}^{\text {req }}=\gamma, \quad \forall i, 0<i<M, i \in I \\
R_{i}^{\text {initial }}(0)=R_{i}^{\text {req }}, \quad \forall i, 0<i<M, i \in I \\
R_{i}^{\text {initial } *}(0)=0, \quad \forall i, 0<i<M, i \in I
\end{gathered}
$$

Some detail simulation procedures of these four scheduling schemes will be given in the following sections.

\section{(1) CBO (Channel-Based Only) Scheduling Scheme}

In $\mathrm{CBO}$ scheduling simulation model, the scheduled user $S_{j}(n)$ in the $n^{\text {th }}$ slot at base station $j$ is expressed as:

$$
S_{j}(n)=\arg \max \left[G_{i j}(n)\right], \quad 0<i \leq M, 0<n \leq N,(i, n) \in I
$$

We assume that mobile terminals are stationary during each run which equals full scheduling duration of 500 slots. It means that the channel condition of each mobile terminal will remain unchanged in distance-dependent only propagation model (i.e, no multipath fading) since the location of each mobile terminal is not changed during a run. Therefore, the mobile terminal with the best channel condition will be always scheduled during 500 slots. When we consider multipath fading in each slot, channel conditions become complex, and channel strength of each user will be affectad considerably regardless of their location. As a result, the mobile terminal which is scheduled in slot $n$ may not be scheduled in next slot $(n+1)$ because of the weakened channel quality caused by deep fading, or the mobile terminal which 
is blocked in slot $n$ may be scheduled in next slot $(n+1)$ because of the intensified channel quality.

In CBO simulation model, we separately accumulate the scheduling frequency that each user has been scheduled during the total simulation time, then we can comprehend that how many users have been scheduled and how many times each user has been scheduled during the total simulation time. Finally, we can compute the average throughput $R_{i}^{*}$ of each mobile terminal and system average throughput $R_{C B O}^{*}$ to evaluate the performance of the scheme.

(2) CB+MA+FS (Channel-Base Mobile-Assisted Fairly-Shared) Scheduling Scheme

In $\mathrm{CB}+\mathrm{MA}+\mathrm{FS}$ scheduling simulation model, the scheduling indicator $Z_{i j}(n)$ for user $i$ in base station $j$ at slot $n$ can be expressed as:

$$
Z_{i j}(n) \doteq\left[H_{i}(n) \times G_{i j}(n)\right], \quad 0<n \leq N, 0<i \leq M,(n, i) \in I
$$

where $G_{i j}(n)$ is the link gain between mobile terminal $i$ and its home base station $j$. Note that $H_{i}(n)$ is the priority factor of user $i$ at slot $n$.

$$
H_{i}(n)=1-\frac{R_{i}^{*}(n-1)}{L R_{i}^{r e q}}
$$

where $R_{i}^{*}(n)$ is the achieved average rate of user $i$ in slot $n$ so far.

In the proposed scheduling scheme, a very important parameter - priority factor $H_{i}$ was introduced. The combined effect of priority factor and real-time channel quality determines which mobile terminal will be scheduled in each slot. Since $H_{i}(n)$ is based on the achieved average rate in slot $n$ so far, the calculation of achieved average rate in each slot is necessary. The transmission rate of the scheduled user which is on the basis of real-time channel condition has to be recorded in each slot.

The process of simulation in specific slot $n$ with combined distance-dependent path loss and multipath fading follows this way:

1. estimate the individual channel quality $G_{i j}(n), \forall i, j$.

2. calculate the individual priority factor $H_{i}(n), \forall i$. 
3. calculate scheduling indicator $Z_{i j}(n), \forall i, j$

4. select the user with the maximum $Z_{i j}(n)$ value to transmit

5. compute the transmission rate $R_{i}(n), \forall i$

6. calculate the achieved average rate $R_{i}^{*}(n+1), \forall i$,

The same procedure will repeat in each slot until the total simulation time is over.

(3) RR (Round Robin) Scheduling Scheme

In RR simulation model, the scheduler cycles the list of active terminals. We assume that total slot number is integer multiple of total user number, then each mobile terminal will be scheduled for the same duration (or has the same scheduling frequency). The scheduled user in RR by base station $j$ at slot $n$ can be expressed as $S_{j}(n)$ under the assumption that total slot number and total user number are same,

$$
S_{j}(n)=n, \quad 0<n \leq N, M=N,
$$

For example, if we assume that the number of total slot is 500 slots, and 100 users are located in each cell, then the scheduled number for each user is

$$
\operatorname{Snum}_{i}=\frac{\text { slots number }}{\text { user number }}=\frac{500}{100}=5
$$

Therefore, each user will be scheduled for 5 times during the total simulation time.

\section{(4) RS (Random) Scheduling Scheme}

In RS simulation model, the channel condition is not considered too as the case in RR. RS selects the mobile terminal to be scheduled randomly in each time slot. The scheduled user in RS by base station $j$ at slot $n$ can be expressed as $S_{j}(n)$ :

$$
S_{j}(n)=\operatorname{random}[\mathrm{M}], 0<n \leq N, n \in I,
$$

In the simulation, the index of scheduled user is created randomly which is a number between 1 and $M$, where $M$ is total user number. 


\subsection{Simulation Results}

Next we evaluate the performance of four scheduling schemes and discuss the simulation results along with the analytical results that were derived in Section 4.3.

\subsubsection{System Throughput}

System throughput is an important parameter in the evaluation of the performance of different scheduling schemes. The system throughput will show if system resources are utilized in the most efficient manner.

In Section 4.3.1, system throughput in scheduling scheme $k$ is defined as,

$$
\begin{aligned}
T_{k} & =\sum_{i=1}^{M} R_{i k}^{*} \\
& =\frac{\sum_{i=1}^{M} \sum_{n=1}^{N} R_{i k}(n)}{N}
\end{aligned}
$$

where $M$ is total number of user, $N$ is total slot number, $k \in\{\mathrm{CBO}, \mathrm{RR}, \mathrm{RS}, \mathrm{CB}+\mathrm{MA}+\mathrm{FS}\}$.

The characteristic of the system throughout is shown in Fig. 5.2 for the two cases of signal attenuation model: (a) distance-dependent only model and (b) distance-dependent combined with Rayleigh fading, for $\mathrm{CBO}, \mathrm{RR}, \mathrm{RS}, \mathrm{CB}+\mathrm{MA}+\mathrm{FS}$ scheduling schemes.

From Fig. 5.2, we confirm that $\mathrm{CBO}$ always has the maximum system throughput among four scheduling schemes since the scheduler in CBO always selects the user which has the best channel condition in each slot. It achieves the highest possible transmission rate in each slot. Hence, the sum of individual current transmission rate will be maximum among four scheduling schemes.

CB+MA+FS has a system throughput which is lower than that of $\mathrm{CBO}$ and higher than that of $R R$ and $R S$. In $C B+M A+F S$, the real-time channel condition is not the only factor to affect scheduling, the achieved average rate and required transmission rate are also considered there. It means that the scheduler will select the user which has relatively good channel condition and high priority factor. Therefore, the relatively high transmission rate is achieved in each slot. 
In $\mathrm{RR}$ and $\mathrm{RS}$, the scheduler does not consider the channel condition. Therefore, the system throughput shows the average situation of the channel conditions.
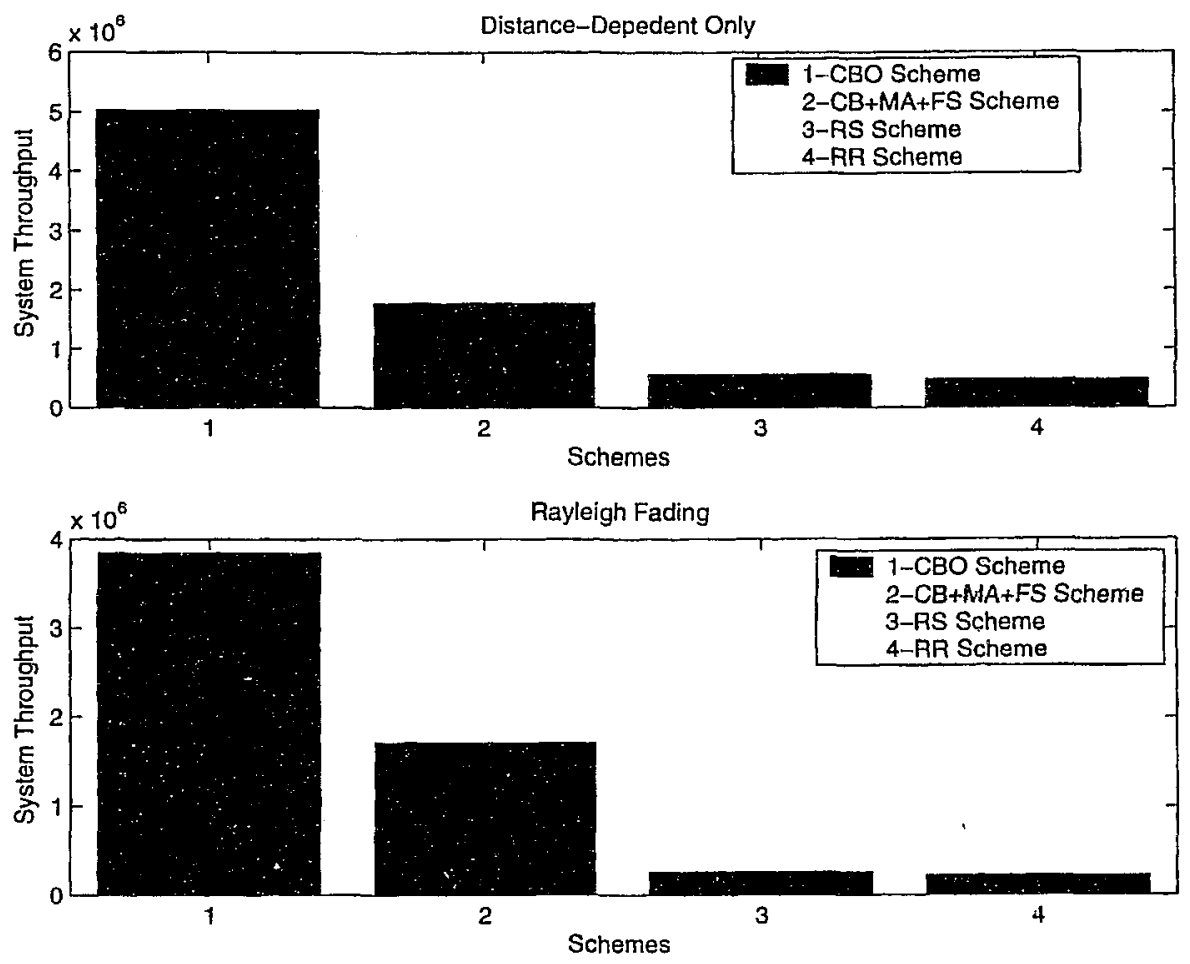

Figure 5.2: System Throughput

We can confirm that the below relationship among these four scheduling schemes for the system throughput using both signal attenuation models.

$$
T_{C B O}>T_{C B+M A+F S}>T_{R S} \approx T_{R R}
$$

This confirms our analytical results discussed in Section 4.3.1.

In Table 5.2, the system throughput for different scheduling schemes under different channel models is recorded.

From Table 5.2, we can find that the the system average throughput is reduced in multipath fading model since Rayleigh fading will weaken the signal from the base station through multipath propagation. In $\mathrm{CBO}$, the system average throughput in multipath fading model 


\begin{tabular}{|c|c|c|c|c|}
\hline Schemes & CBO & CB+MA+FS & RS & RR \\
\hline Distance-Dependent Only (kbps) & 5022.5 & 1768.4 & 572.3 & 487.8 \\
\hline Rayleigh Fading (kbps) & 3844.3 & 1712.1 & 260.6 & 224.5 \\
\hline Reduction Percentage \% & 24 & 5 & 54 & 55 \\
\hline
\end{tabular}

Table 5.2: System Throughput.

is decreased by almost $24 \%$ compared to distance-dependent only model which is much larger than the reduction percentage in $\mathrm{CB}+\mathrm{MA}+\mathrm{FS}$ ( $5 \%$ ), and is smaller than that in RS (54\%) and RR (55\%). Therefore, we can conclude that any fading model will affect CBO more evidently than the other three scheduling schemes since the channel condition is the only consideration in $\mathrm{CBO}$.

We also find that in distance-dependent only path loss condition, CBO has almost 10 times of the system throughput in RR and RS and 3 times of the system throughput in $\mathrm{CB}+\mathrm{MA}+\mathrm{FS}$; in multipath fading condition, $\mathrm{CBO}$ has almost 15 times of the system throughput in RR and RS and 2 times of the system throughput in CB+MA+FS. CBO provides efficient data transmission advantage in system throughput among these four scheduling schemes. However, as we will see next, it has problem with fair allocation of individual throughput.

\subsubsection{Standard Deviation of Individual Throughput}

Because the fairness is an important improvement in our proposed scheduling scheme compared to CBO as noted through analytical means in Section 4.3.2, we evaluate the fairness of $\mathrm{CBO}, \mathrm{RR}, \mathrm{RS}, \mathrm{CB}+\mathrm{MA}+\mathrm{FS}$ using standard deviation of individual throughput which was defined in Section 4.1.1. In Fig. 5.3, standard deviation of individual throughout for two propagation models using $\mathrm{CBO}, \mathrm{RR}, \mathrm{RS}, \mathrm{CB}+\mathrm{MA}+\mathrm{FS}$ scheduling schemes is shown.

From the figure, we can find that $R R$ always has minimum standard deviation of individual average throughput since each mobile terminal gets the same priority level to be scheduled during simulation period. It means that each mobile terminal will have equal 
frequency of scheduling if total slot number is integer times of the total user number in each cell as can be seen in Fig. 5.6. RR is an absolutely fair scheduling scheme because most individual throughput are concentrated to the system throughput.
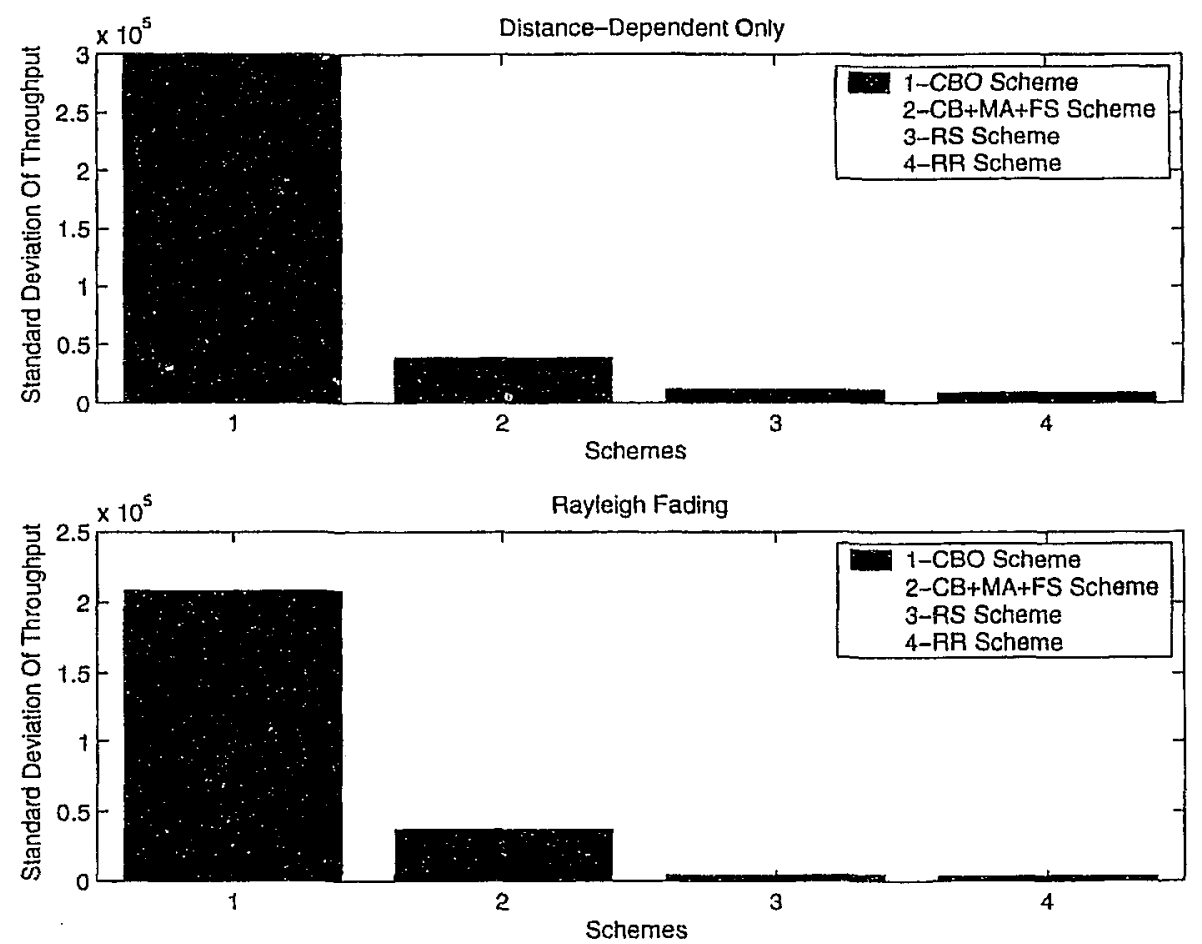

Figure 5.3: Standard Deviation of Individual Throughput.

$\mathrm{RS}$ is a partial fair scheduling scheme since the scheduler selects the mobile terminal to be scheduled randomly at each time slot. During a short scheduling period, some mobile terminals will get more chances to be scheduled and the others will get less. The frequency of scheduling for each user is shown in Fig. 5.6. In RS scheduling scheme, each user has different frequency of scheduling. However, if during a long scheduling period, all the users will have almost equal chances to be scheduled as in RR. From Fig. 5.3, we find that RS has small standard variance of individual throughput which is a little larger than that of RR corresponding to our analysis in Section 4.3.2.

$\mathrm{CBO}$ is the scheduling scheme which does not take the fairness into account. We find 


\begin{tabular}{|c|c|c|}
\hline Schemes & Distance-Dependent Only (kbps) & Rayleigh Fading (kbps) \\
\hline $\mathrm{CBO}$ & 300.0 & 208.0 \\
\hline $\mathrm{CB}+\mathrm{MA}+\mathrm{FS}$ & 38.8 & 37.4 \\
\hline $\mathrm{RS}$ & 11.9 & 4.5 \\
\hline $\mathrm{RR}$ & 9.0 & 3.9 \\
\hline
\end{tabular}

Table 5.3: Standard Deviation of Individual Throughput

that $\mathrm{CBO}$ has the worst fairness performance which has the maximum standard deviation of individual throughput shown in Fig. 5.3. Therefore, we know that the average throughput of individual user is distributed dispersedly in CBO.

$\mathrm{CB}+\mathrm{MA}+\mathrm{FS}$ has the larger standard deviation of throughput compared to $\mathrm{RR}$ and RS, but smaller standard deviation than that of $\mathrm{CBO}$, since it considers the fairness into account. Therefore, the distribution of individual throughput in CB+MA+FS is relatively concentrated compared to CBO, and is relatively dispersed compared to RR and RS.

In Table 5.3, the standard deviation values for different scheduling schemes for two propagation models are recorded.

We can confirm the below relationship among these four scheduling schemes for the standard deviation of individual throughput (or fairness) in both signal propagation conditions. This also confirms our analytical results discussed in Section 4.3.2.

$$
F_{C B O}>F_{C B+M A+F S}>F_{R S} \approx F_{R R}
$$

It can be noted that Rayleigh fading reduces the standard variance as compared to distancedependent only.

\subsubsection{Frequency of Scheduling}

Frequency of scheduling is defined as how many times that each user is scheduled during total simulation period.

CBO Scheduling Scheme 
In CBO scheme, minority of mobile terminals which have the best channel conditions were scheduled while majority of mobile terminals could not be scheduled. Especially in distancedependent path loss only model, only 2 users are scheduled during the simulation period in a two-cell system as shown in Fig. 5.4. CBO is a kind of absolute non-fairness scheduling since the mobile terminal with the best channel condition is always scheduled while the rest of the mobile terminals are delayed. In multipath fading model, there are 9 more mobile terminals are scheduled during the simulation period as shown in Fig. 5.5, because that the individual channel condition may be changed in each slot because of Rayleigh fading. But most users are still not scheduled since we have a total of 200 users in two-cell system.

\section{CB+MA+FS Scheduling Scheme}

$\mathrm{CB}+\mathrm{MA}+\mathrm{FS}$ is the proposed scheduling scheme which considers both the channel condition of each mobile terminal as well as fairness allocation of individual throughput. In $\mathrm{CB}+\mathrm{MA}+\mathrm{FS}$, there are much more users being scheduled in both signal propagation models as shown in Fig. 5.4 and Fig. 5.5 compared to CBO.

Next we will discuss frequency of scheduling in $C B+M A+F S$ in both signal attenuation models.

Distance-dependent model: There are 21 more users scheduled in CB+MA+FS compared to $\mathrm{CBO}$. We can find that each user has different frequency of scheduling and these frequency are in the range of 0 to 87 (see Fig. 5.4). It means that the maximum frequency of scheduling is 85 which is sinaller compared to 500 of CBO. We already know that the channel condition of distance-dependent only model is only related to the distance between the mobile terminal and its home base station. That is, if the mobile terminal is very close to its base station, it has the best channel quality which is a much larger magnitude compared to those of the other mobile terminals since we use uniformly distributed to locate individual user in each cell. Therefore, when we schedule the users, the channel condition becomes the dominant factor to effect the scheduler although we introduce priority factor which is adjusted based on the updated achieved average rate of each user in each slot. The value of scheduler is effected mostly by the channel condition. This is the reason that the most users can not 

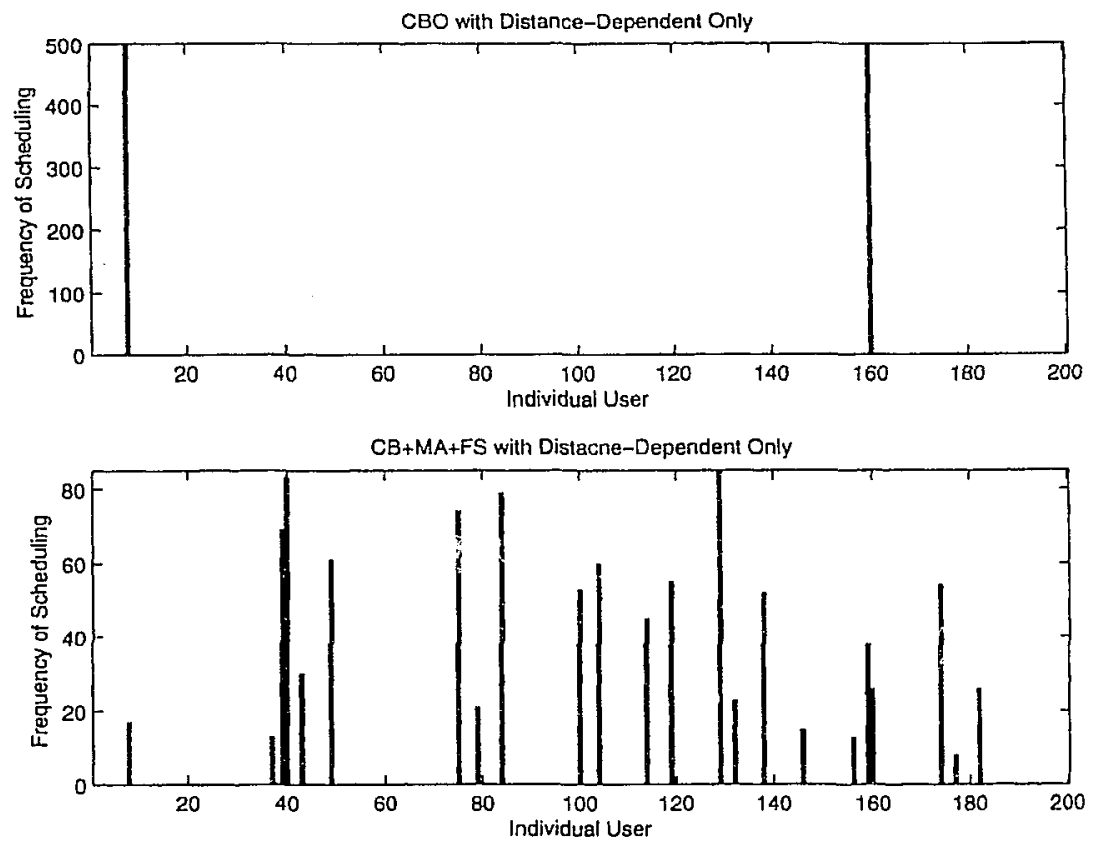

Figure 5.4: Frequency of Scheduling for Individual User with Distance-Dependent Only.

be scheduled frequently and the mobile terminals which are closer to base station will be scheduled during most slots duration in the distance-dependent only model.

Multipath fading model: There are 63 more users scheduled in CB+MA+FS compared to $\mathrm{CBO}$, and individual frequency of scheduling is in the range of 0 to 52 (see Fig. 5.5). That is, the difference of individual frequency of scheduling gets smaller, and the distribution of scheduling frequency gets more even. The fairness of scheduling in CB+MA+FS improves evidently since Rayleigh fading affects the channel condition and makes channel conditions more complex. The more complex the channel conditions are, the more active the scheduler is. In distance-dependent only model we already noted that the channel condition is the dominant factor to affect the scheduler. Combined with Rayleigh fading, the channel condition becomes complex; for example, the mobile terminal which is extremely close to its base station may undergo deep fading or the mobile terminal which is far away from its home base station may achieve high channel gain. In our simulation, the channel condition (with Rayleigh fading) is varied in each time slot although their location is fixed. Therefore, the 
dominant effect from individual location is weakened, the scheduler in CB+MA+FS becomes more active.
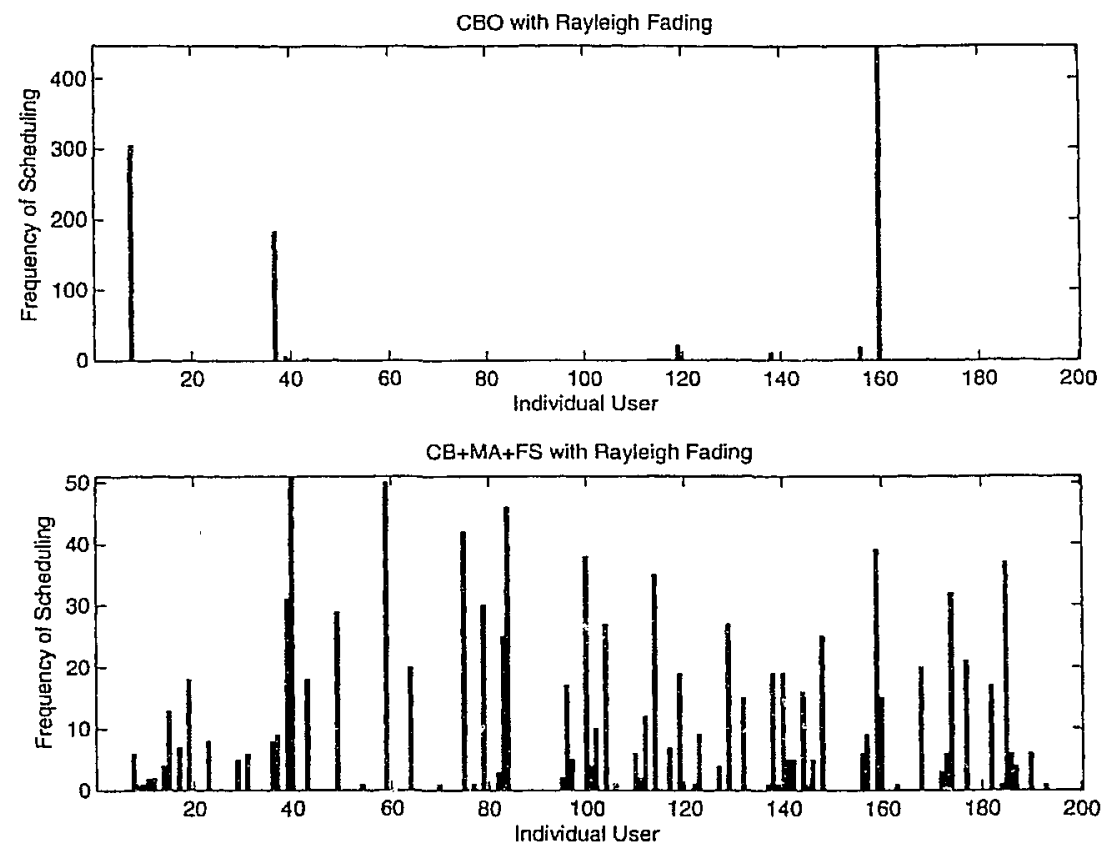

Figure 5.5: Frequency of Scheduling for Individual User with Rayleigh Fading (CBO and $\mathrm{CB}+\mathrm{MA}+\mathrm{FS})$.

Note that,

1. Due to multipath signal propagation, channel conditions vary considerably over time; hence, more users get scheduled as shown in Fig. 5.5

2. Rayleigh fading increases the activity of $C B+M A+F S$ scheduler as compared to distancedependent only.

\section{RS Scheduling Scheme}

In $\mathrm{RS}$, the distribution of scheduling frequency is relatively even compared to $\mathrm{CBO}$ and $\mathrm{CB}+\mathrm{MA}+\mathrm{FS}$ as shown in Fig. 5.6 since the maximum frequency of scheduling is 11 and the difference of scheduling frequency gets more smaller. We find that almost each user 
is scheduled in RS during simulation period. We can expect that RR will also be as fair scheduling as that of $R R$ if simulation period is long enough.

\section{RR Scheduling Scheme}

In $\mathrm{RR}$, the distribution of selection rate is absolutely even as shown in Fig. 5.6 since each user has equal frequency of scheduling, i.e., 5 in our case ( total simulation time is 500 slots, and 100 users in each cell), and all the users are scheduled during simulation period.
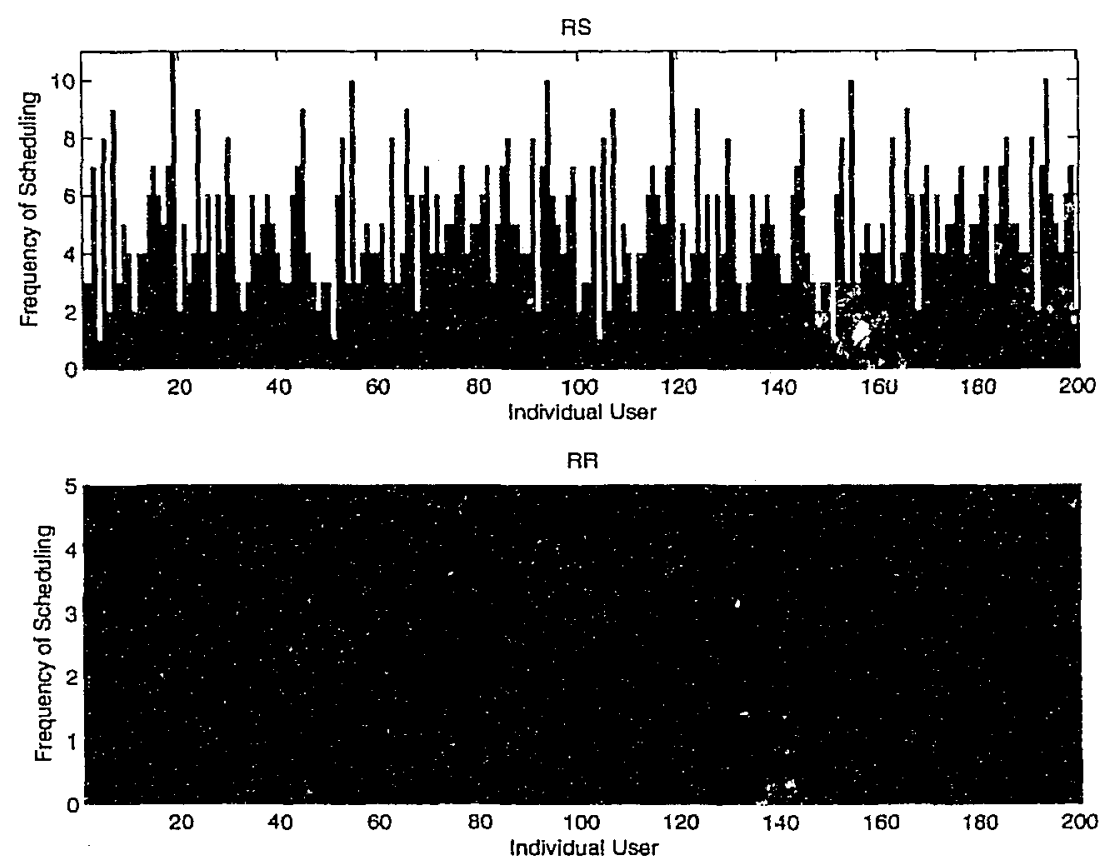

Figure 5.6: Frequency of Scheduling for Individual User with Rayleigh Fading (RR and RS).

In Table 5.4, we summarize the percentage of users scheduled using different scheduling schemes. CB+MA+FS increases the even distribution of individual scheduling frequency evidently compared to CBO, but still is lower than that of RS and RR in which almost each user gets a chance to be scheduled during simulation period. 


\begin{tabular}{|c|c|c|}
\hline Schemes & Distance Dependent Only (\%) & Rayleigh Fading (\%) \\
\hline CBO & 1 & 5.5 \\
\hline $\mathrm{CB}+\mathrm{MA}+\mathrm{FS}$ & 11.5 & 37 \\
\hline $\mathrm{RS}$ & 99 & 99 \\
\hline $\mathrm{RR}$ & 100 & 100 \\
\hline
\end{tabular}

Table 5.4: Percentage of Users Scheduled.

\subsubsection{Individual Throughput}

Even distribution of individual scheduling frequency does not necessarily mean even allocation of individual throughput. Let us consider the individual throughput next.

We now discuss individual throughput in multipath fading model (i.e., Rayleigh fading ). System throughput (as discussed in Section 5.2.1) shows how the radio resources are
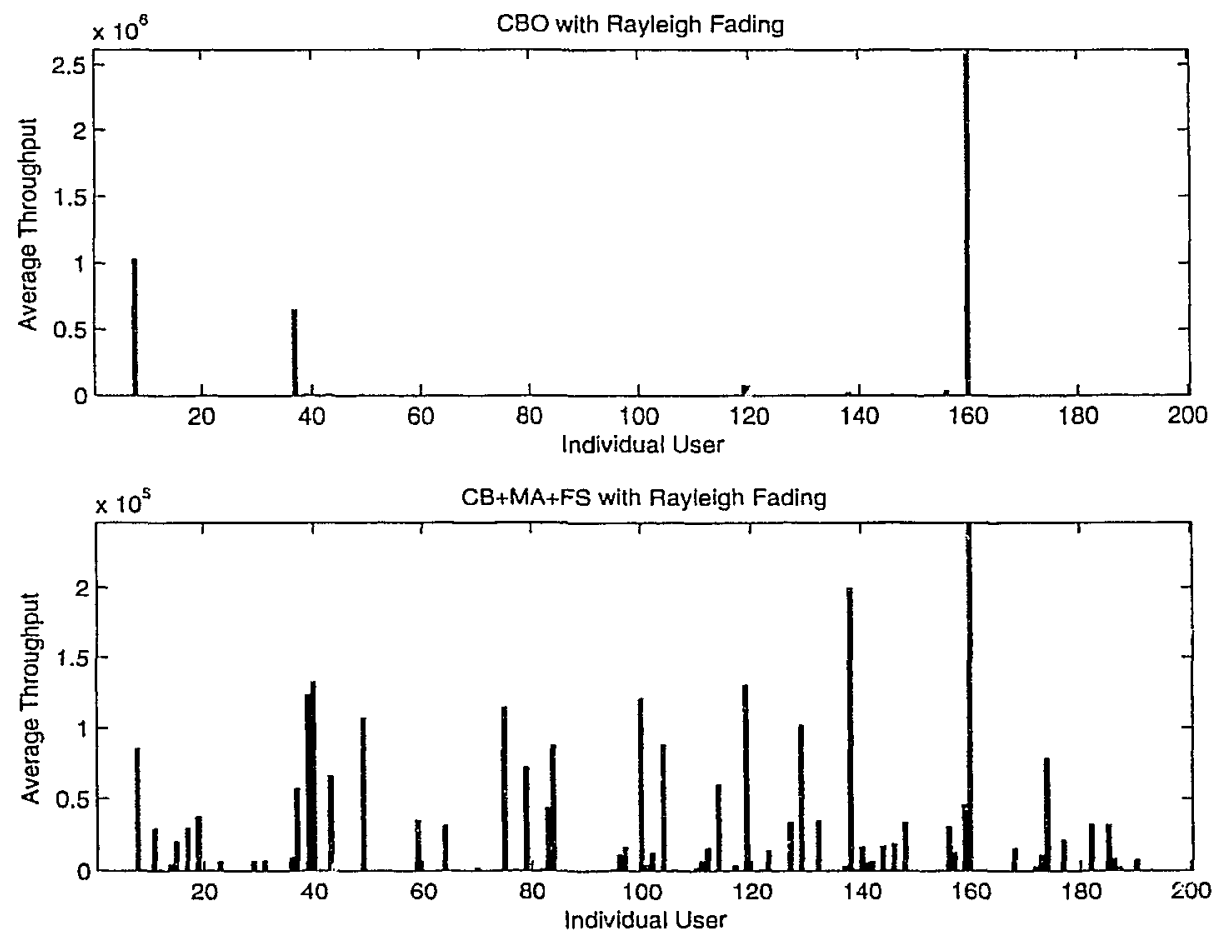

Figure 5.7: Individual Throughput with Rayleigh Fading ( $\mathrm{CBO}$ and $\mathrm{CB}+\mathrm{MA}+\mathrm{FS}$ ). 
utilized, either efficiently or not. Individual throughput shows how individual user utilizes radio resources. If the radio resources are allocated to individual user efficientily, each user can achieve high average throughput, and vice versa. Therefore, in CDMA systems, the main task is to maintain high system throughput while assigning relatively high individual throughput in order to obtain high QoS and utilize system resources efficiently.

In Fig. 5.7, we note that the scheduled users in CBO have very high average throughput in magnitude of $\mathrm{Mbps}$, but system throughput is just allocated to a few users, the distribution of radio resources allocation is uneven since most users achieve zero average throughput.

In RR and RS as shown in Fig. 5.8, a few scheduled users get high average throughput in tens of $\mathrm{kbps}$, and most scheduled use:s achieve very low average throughput in kbps. Although the distribution of scheduling frequency in RR and RS is even, the individual average throughout is verv low, and it can not satisfy the individual transmission demand.
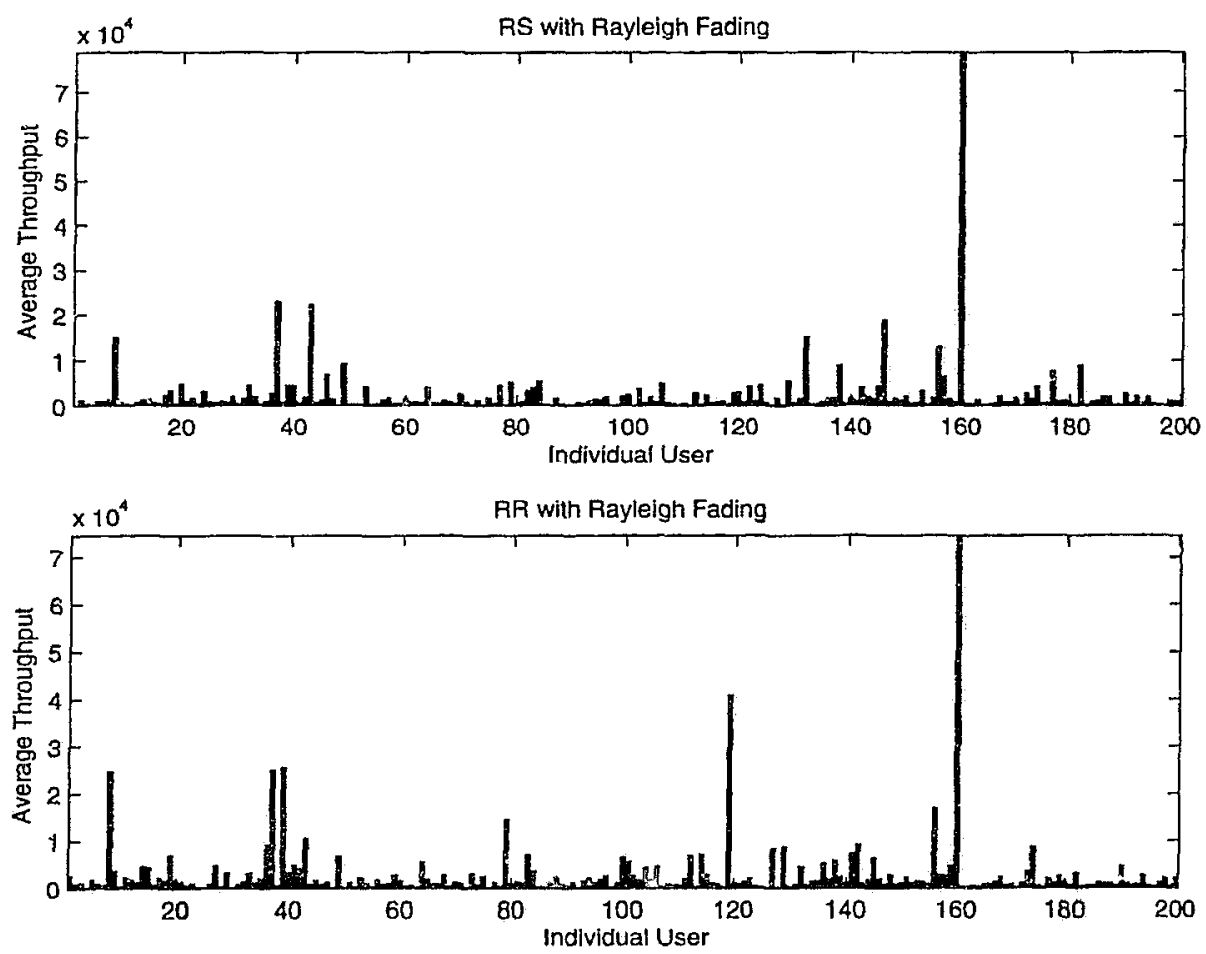

Figure 5.8: Individual Throughput, with Rayleigh Fading (RR and RS). 
$\mathrm{CB}+\mathrm{MA}+\mathrm{FS}$ has relatively higher individual average throughput compared to RS and $\mathrm{RR}$ and lower than that of CBO. In Fig. 5.7, we find that only $10 \%$ of the scheduled users average throughput is in hundreds of bits and the rest is in the magnitude of tens of kbps. Therefore, CB +MA+FS not only increases the evenness of the system resource allocation compared to $\mathrm{CBO}$, but also raises the efficiency of system resources utilization compared to RS and RR.

Among $\mathrm{CBO}, \mathrm{RS}$, and $\mathrm{RS}$, the individual average rate is only related to the real-time channel condition of the scheduled user, but in CB+AM+FS, the individual average rate is adjusted through system tolerance factor $L$ and required transmission rate $R_{i}^{r e q}$. If the average achieved rate of the scheduled user $i$ equals $L R_{i}^{\text {req }}$ in specific slot, the user $i$ will be blocked in next slot since its priority equals 0 , and the user with relatively good channel condition will be scheduled since the scheduler considers the combined effect of real-time channel condition and priority factor. Therefore, the user with the best channel condition can not keep the transmission always and the user with the worst channel condition can get the chance to be scheduled; that is the reason that $C B+M A+F S$ has lower individual average throughput than CBO and higher individual average throughput than RS and RS.

\subsubsection{System Tolerance Factor - L}

In section 4.3.3, we discussed some characteristics of the system-dependent tolerance factor $L$ which determines the maximum range the individual average rate can be achieved over time.

Through simulation study, we obtain some results which are shown in Table 5.5 that describes the sensitivity of $L$ towards the system throughput and standard deviation of individual throughput. We also plot the relationship between the value of $L$ and system throughput in Fig. 5.9 , and the relationship between the value of $L$ and standard deviation of individual throughput in Fig. 5.10.

Fig. 5.9 and Fig. 5.10 show the trade off between achieving high system throughput (i.e., 

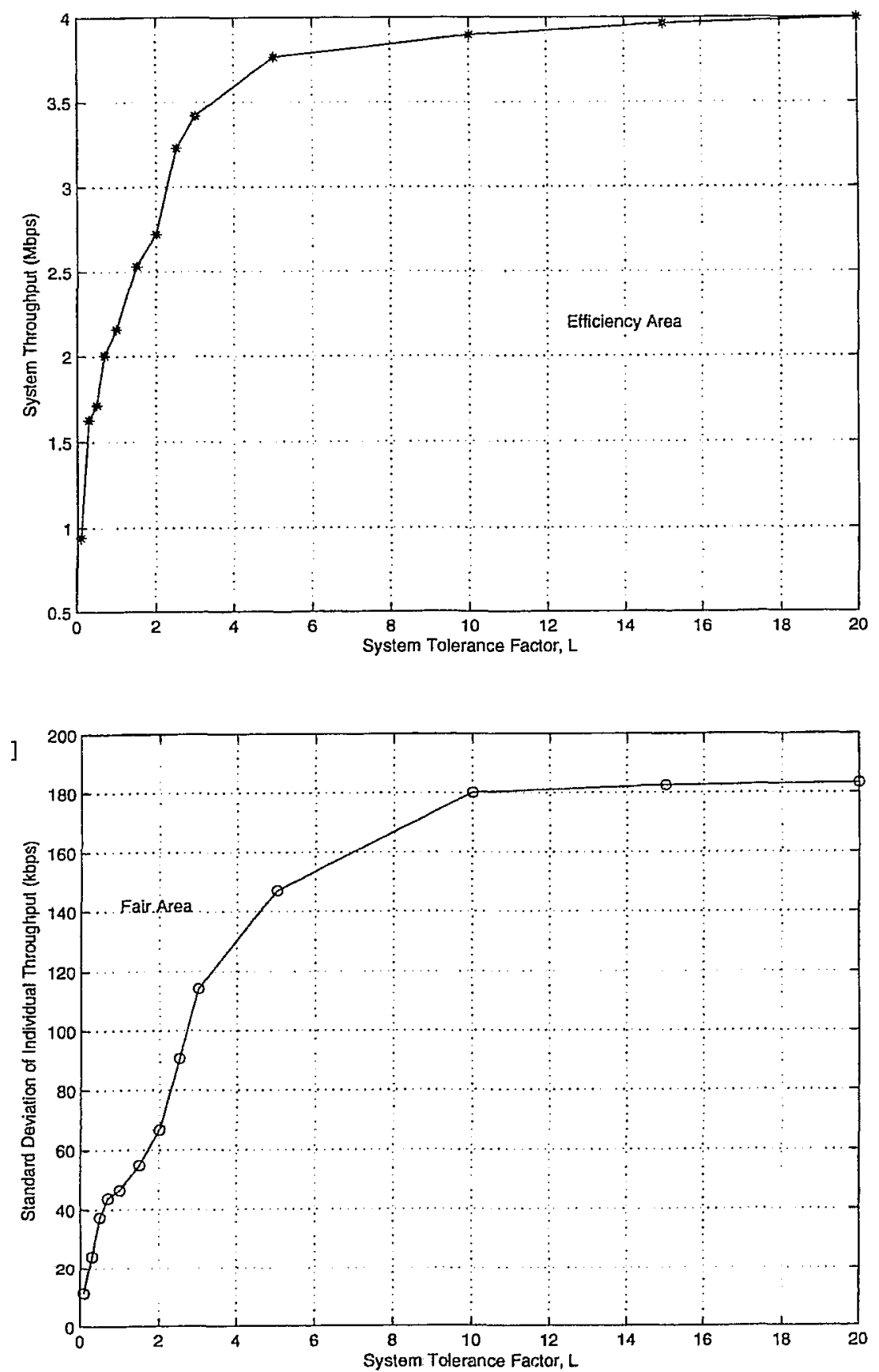

Figure 5.10: Standard Deviation of Individual Throughput versus L with Rayleigh Fading.

Reproduced with permission of the copyright owner. Further reproduction prohibited without permission. 


\begin{tabular}{|c|c|c|}
\hline $\mathrm{I}$ & System Throughput (Mbps) & Standard Deviation of Throughput (kbps) \\
\hline 0.1 & 0.9415 & 11.3 \\
\hline 0.3 & 1.6263 & 24.2 \\
\hline 0.5 & 1.7124 & 37.4 \\
\hline 0.7 & 2.0050 & 43.7 \\
\hline 1.0 & 2.1579 & 46.4 \\
\hline 1.5 & 2.5317 & 54.8 \\
\hline 2.0 & 2.7209 & 66.6 \\
\hline 2.5 & 3.2282 & 90.6 \\
\hline 3.0 & 3.4189 & 114.2 \\
\hline 5.0 & 3.7662 & 147.0 \\
\hline 10 & 3.8971 & 180.1 \\
\hline 15 & 3.9628 & 182.5 \\
\hline 20 & 3.9974 & 183.3 \\
\hline
\end{tabular}

Table 5.5: System Throughput and Standard Deviation of Individual Throughput with $L$ in Rayleigh Fading

efficiency) and low standard deviation of individual throughput (i.e., fairness) in a wireless system.

We can make some observations through the simulation results that were also observed analytically in section 4.3 .3 .

1. System average throughput will be increased with $L$ since the larger the value of $L$, the larger the maximum allowable achieved individual average throughput allowed at any time; hence, the longer scheduling time for a user with the best channel condition, as a result, the higher system average throughput is achieved.

2. The degree of fairness will be decreased with $L$ since the smaller the value of $L$, the smaller the maximum allowable achieved individual average throughput; hence, faster the over-scheduled user is blocked, as a result, more users are scheduled and achieve high degree of fairness in using the system resources.

3. $L$ affects the activity of the scheduler, and determines the degree of fairness. We have 
found that, (a) if $L<1$, the scheduler works very well in terms of fair allocation of system throughput to individual users, (b) if $L>1$, the scheduler is not so active, and the less users are scheduled, (c) if $L>10$, the scheduling operation is almost the same as $\mathrm{CBO}$ in which the system throughput is maximized.

In short, it can be said that the value of $L$ affects the degree of fairness, and determines how tolerant the system is with respect to allocation of achieved average rate to individual user and also to the system.

\subsubsection{SIR}

We estimate the SIR of the scheduled mobile terminals in each time slot during the simulation period and record them in ascending order, then, gei the SIR distribution of scheduled individual for four scheduling schemes.

Fig. 5.11 shows SIR distribution of CBO. In CBO, the scheduler always chooses the user with the maximum link gain in each slot. Therefore, CBO will achieve maximum SIR in each slot.

Fig. 5.12 shows SIR distribution of CB+MA+FS. We can find that the SIR in CB+MA+FS is higher compared to RR and RS, but lower than that of CBO. In CB+MA+FS, the scheduler selects the user based on the combined effect of channel condition and priority factor. So the channel condition of the scheduled user can not be always best and it is can not be worst too. This is the reason that $C B+M A+F S$ has better signal quality than that of $R R$ and $\mathrm{RS}$, while it has worse signal quality than that of $\mathrm{CBO}$.

Fig. 5.13 and Fig. 5.14 show SIR distribution separately for RS and RR. The SIR in RS and RR are relatively lower than that of $C B O$ and $C B+M A+F S$. In RR and RS, the scheduler chooses the mobile terminal to be scheduled with no consideration of channel quality, so it can be good or bad. 

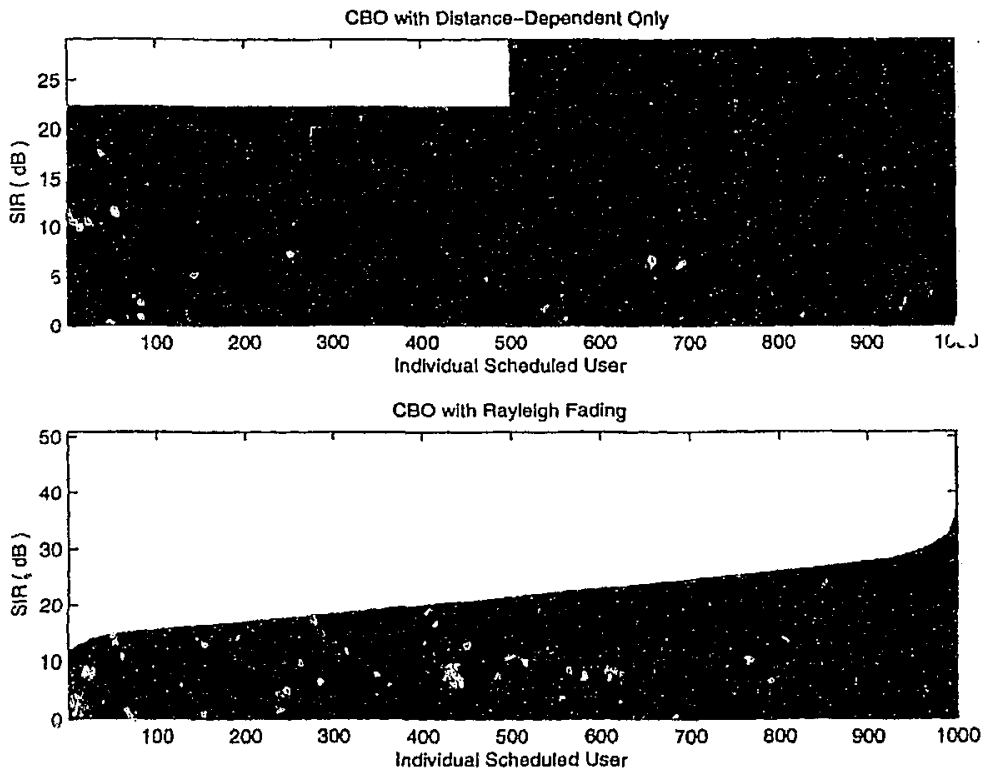

Figure 5.11: SIR Distribution in CBO
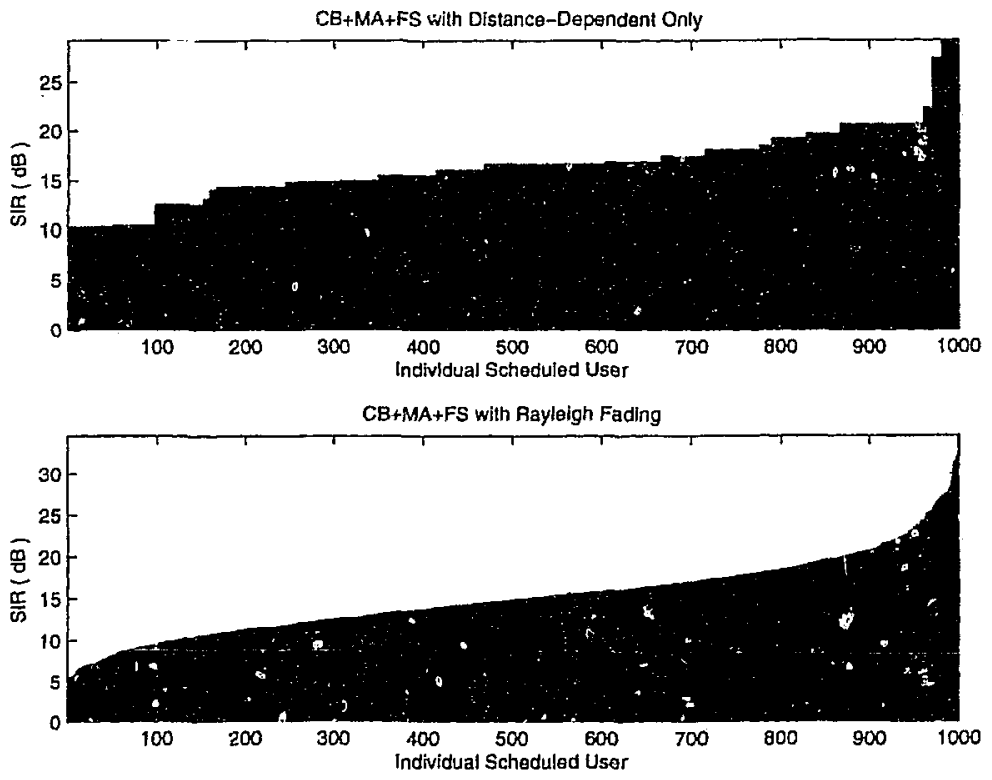

Figure 5.12: SIR Distribution in $C B+M A+F S$

Reproduced with permission of the copyright owner. Further reproduction prohibited without permission. 

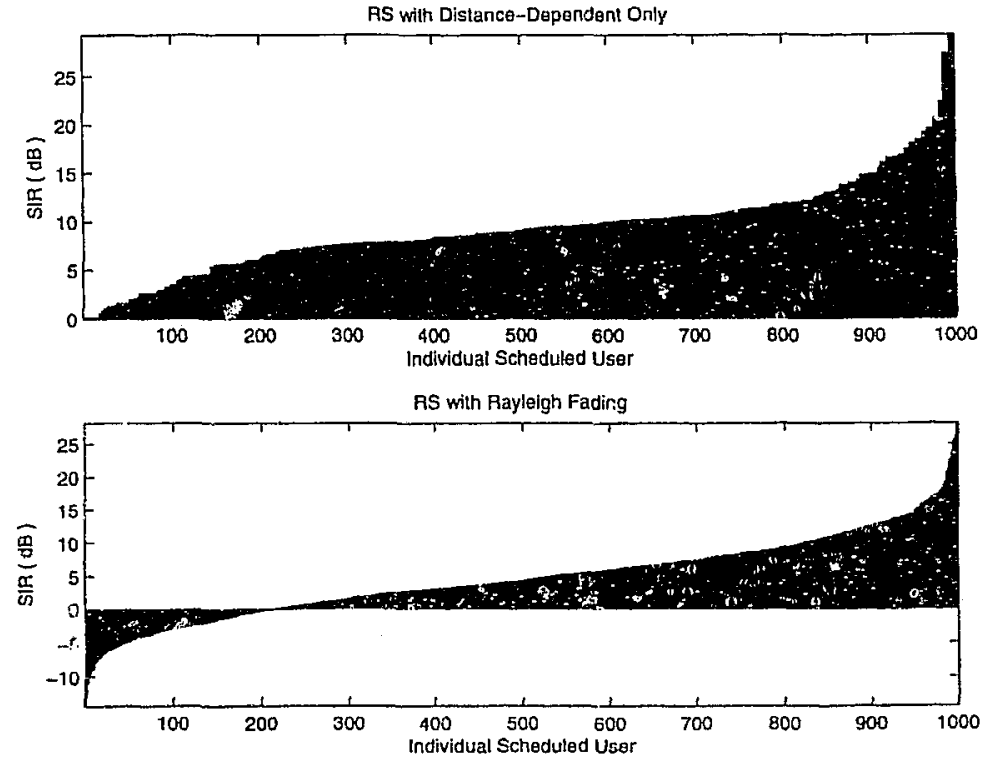

Figure 5.13: SIR Distribution in RS.
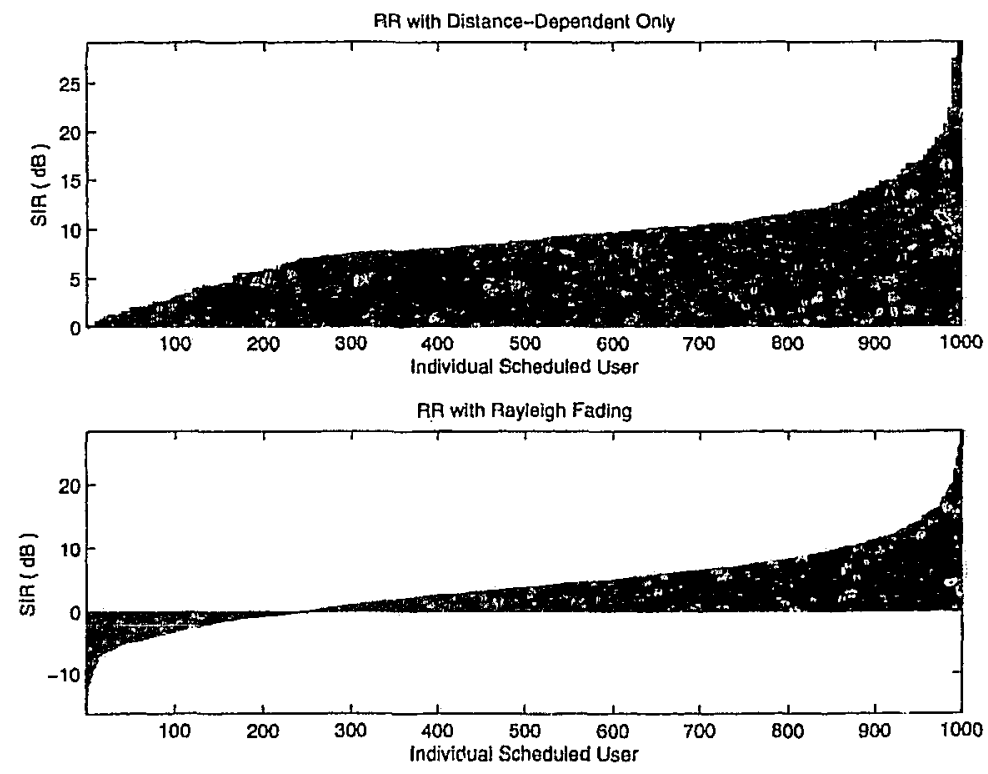

Figure 5.14: SIR Distribution in RR.

Reproduced with permission of the copvright owner. Further reproduction prohibited without permission. 


\begin{tabular}{|c|c|c|}
\hline Schemes & Distance-Dependent Only (dB) & Rayleigh Fading (dB) \\
\hline CBO & 25.8 & 21.6 \\
\hline CB+MA+FS & 16.5 & 15.2 \\
\hline RS & 9.4 & 4.8 \\
\hline RR & 9.1 & 4.1 \\
\hline
\end{tabular}

Table 5.6: Average SIR in a Two-cell System

\begin{tabular}{|c|c|c|c|c|}
\hline & CB0 & CB+MA+FS & RS & RR \\
\hline Fairness & Worst & Good & Better & Best \\
\hline System Throughput & Highest & High & Low & Low \\
\hline Distribution of Scheduling Frequency & Uneven & Even & More even & Most even \\
\hline Signal Quality & Best & Good & Bad & Bad \\
\hline Efficiency of Resources Utilization & Highest & High & Low & Low \\
\hline
\end{tabular}

Table 5.7: Overall Summary of Performance Measures in CBO, $C B+M A+F S, R R, R S$ Scheduling Schemes.

Average SIR for four scheduling schemes is shown in Table 5.6.

Finally, we can conclude that the SIR in distance-dependent only is better than the SIR in multipath propagation because of the effect of fading; CBO has the best system signal quality during simulation, $C B+M A+F S$ has relative good system signal quality, SIRs in $\mathrm{RR}$ and $\mathrm{RS}$ are worse than in $\mathrm{CBO}$ and $\mathrm{CB}+\mathrm{MA}+\mathrm{FS}$. We have the following relationship:

$$
S I R_{C B O}>S I R_{C B M A F S}>S I R_{R R, R S}
$$

Simulation results confirm this analytical observation.

\subsection{Conclusions}

We finally tabulate our results for different scheduling schemes in Table 5.7. These results are based on simulation studies and also supported analytically. Therefure, we make the following conclusions. 
1. $\mathrm{CB}+\mathrm{MA}+\mathrm{FS}$ provides more fair share of radio resources to individual users compared to $\mathrm{CBO}$ for different high speed data rate traffic.

2. In $\mathrm{CB}+\mathrm{MA}+\mathrm{FS}$, frequency of scheduling for individual user is increased evidently compared to $\mathrm{CBO}$. Therefore, more users get chances to transmit, and the number of users being blocked is reduced simultaneously.

3. $\mathrm{CB}+\mathrm{MA}+\mathrm{FS}$ achieves more even distribution of individual average throughput in terms of smaller standard deviation of individual average throughput compared to CBO. The difference between system average throughput and individual average throughput is reduced evidently. The requirements of individual mobile terminal are satisfied better, and the system resource is allocated in more fair manner because the more flexible adjustment of the unevenness of throughput per mobile terminal is permitted in $C B+M A+F S$ compared to $C B O$.

4. $\mathrm{CB}+\mathrm{MA}+\mathrm{FS}$ improves signal quality evidently in terms of SIR compared to $\mathrm{RR}$ and RS. The scheduled user can obtain higher SIR at each slot in $C B+M A+F S$ than that in $R R$ and $R S$, since the channel condition is one of the considered factors in CB+MA+FS.

5. Scheduler is sensitive to system tolerance factor $L$. Along with the decrease of $L$ value, the maximum allowable range of individual achieved average rate gets smaller, the scheduled period of individual user gets shorter, and more users are scheduled, finally degree of fairness is increased. 


\section{Chapter 6}

\section{Summary and Future Extensions}

\subsection{Summary}

Future wibeband CDMA systems are required to efficiently utilize the limited radio spectrum due to the rapidly growing demands for high data rate services. Being one of the most important components of quality of service provisioning and resource sharing, packet scheduling plays a key role in wireless data networks.

In our work, we have discussed the problem of resource sharing in mobile communication system. We discussed the existing conventional scheduling schemes in terms of scheduler operation, efficient spectral resources usage and degree of fairness.

We proposed a fair packet scheduling algorithm to achieve fair individual throughput while maintaining the relatively high system average throughput for high data transmission application in CDMA down link systems. We defined the fairness and introduced the priority factor to take fairness into account that was based on individual service requirements, and achieved throughput. We did an analysis about system throughput and standard variance of individual throughput, and expounded the characteristic of system tolerance factor $L$ which determines the tolerance of the scheduling system towards allocating additional data rate compared to the required rate and affects the degree of fairness. We showed that the proposed algorithm achieves both good individual throughput and good system throughput.

Finally, we compared the performance between the proposed fair scheduling algorithm and the existing conventional scheduling schemes through simulation studies and confirmed 
the effectiveness of the proposed $C B+M A+F S$ scheduling scheme and the fact that the effectiveness of system resource allocation is improved while maintaining the high efficiency of system resource utilization.

\subsection{Future Work and Practical Implementation}

Although the application we considered in simulation and numerical examples focused on high data packet transmission, this algorithm can apply equally well to other multimedia. transmission services, such as voice and video. Voice and video are real-time traffic and thus have strict transmission delay requirements. However, they can tolerate a certain degree of transmission errors. On the other hand, data traffic is non-real time in nature but requires high transmission accuracy. The transmission delay requirement depends on each particular data application. Hence, in the proposed scheme, we can introduce another service priority factor to determine different priority of diverse traffic. Because of the tolerance of some degree of transmission errors, voice and video traffic can be transmitted in the adverse channel conditions if needed. Therefore, the scheduling scheme can consider the real-time channel quality, priority for achieved average rate, and service priority.

In our simulation, we only considered two propagation models: free space and Rayleigh fading, and in fact, for most mobile practical radio channels, it is much more complex. So, in the future work, we can consider more factors, such as shadow fading and mobility of mobile terminals.

$\mathrm{CB}+\mathrm{MA}+\mathrm{FS}$ has the most compley ty among these four scheduling schemes since the scheduler in base station needs to calculate and record many parameters, such as: channel conditions, achieved average rates, current transmission rates and priority factors. In each slot, the scheduler has to compare the parameter value and select the user to be scheduled. All these operations increase the complexity in base station and raise the cost finally. 


\section{Bibliography}

[1] R. Esmailzadeh and M. Nakagawa, "TDD-CDMA for Wireless Communications," Artech House Inc., Artech House Universal Personal Communications Series, 2002.

[2] V. Garg, "IS-95 CDMA and CDMA200C," Pearson Education Ir:", 2002.

[3] G. Mandyam and J. Lai, "CDMA Systems for Enhanced Data Services," Academic Press, 2002.

[4] T. Rappaport, "Wireless Communications," Prentice Hall, 2002.

[5] A. Salmasi, "CDMA IS-95 for Cellular and PCS," McGraw-Hill Professional, 2000.

[6] P. Bender, et. al., "CDMA/HDR: A Bandwidth-Efficient High-Speed Wireless Data Services for Normadic Users," Communications Magazine, Volum: 38, pp. 70-77, 2000.

[7] R. Love, et. al., "High Speed Downlink Packet Access Performance," Vehicular Technology Conference, Volume: 3, pp. 2234-2238, 2001.

[8] A. Jalali, R. Padovani and R. Pankaj, "Data Throughput of CDMA-HDR a High Efficiency-High Data Rate Personal Communication Wireless System," Vehicular Technology Conference Proceedings, Volume: 3, pp. 1854-1858, 2002.

[9] Y. Choi and Y. Han, "A Channel-based Scheduling Algorithm for CDMA2000 1*EVDO System," Personal, Indoor and Mobile Radio Communications, Volume: 5, pp. 2259-2263, 2002. 
[10] K. Kim and H. Kim, "A Proportional Fair Scheduling Algorithm with QoS and Priority in 1*EV-DO," Personal, Indoor and Mobile Radio Communications, Volume: 5, pp. $2239-2243,2002$.

[11] A. Yamaguchi and Y. Takeuchi, "Forward link Packet Scheduler for High-speed Packet Data System," Personal, Indoor and Mobile Radio Communications, Volume: 5, pp. 2160-2164, 2002.

[12] H. Fattah and C. Leung, "An Overview of Scheduling Algorithms in Wireless Multimedia Networks," IEEE Wireless Communications, Volume: 9, pp. 76-83, 2002.

[13] V. Huang and W. Zhuang, "Optimal Resource Management in Packet-Switching TDD CDMA Systems," Personal Communications, Volume: 7, pp. 26-31, 2000.

[14] B. Sklar, "Rayleigh Fading Channels in Mobile Digital Communication Systems," IEEE Communications Magazine, Volume: 35, pp. 102-109, 1997.

[15] A. Yamaguchi and Y. Takeuchi "Multicarrier Forward Link Packet Scheduler for Highspeed Packet Data System," Personal, Indoor and Mobile Radio Communications, Volume: 5, pp.2160-2164, 2002.

[16] S. Borst and K. Ramanan "Downlink Scheduling in CDMA Data Networks," GLOBECOM'99, Volume: 5, pp. 2653-2657, 1999.

[17] Y. Choi and S. Bahk "QoS Scheduling for Multimedia Taffic in Packet Data Cellular Networks," IEEE ICC'03, Volume: 1, pp. 358-362, 2003.

[18] S. Lu, V. Bharghavan and R. Skikant "Fair Scheduling in Wireless Packet Networks," IEEE/ACM Trans. on Networking, Volume: 7, pp. 473-489, 2000.

[19] M. Kang and S. Wilbur "A Fair Guaranteed Down-link Sharing Scheme for Cellular" Packet Switched Networks," IEEE Global Telecommun., Volume: 2, pp. 1006-1010, 1999.

Reproduced with permission of the copyright owner. Further reproduction prohibited without permission. 
[20] L. Mailaender, H. Huang and H. Viswanathan "Simple Inter-Cell Coordination S: iremes for a High Speed CDMA Packet Downlink," IEEE Vehicular Technology Conference Proceedings, Volume: 9, pp. 1845-1848, 2000. 